ANL -6318

MASTER ANL-6318

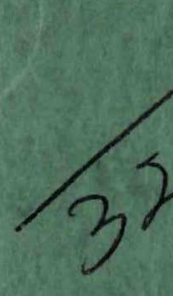

176

Argonne Rational Laboratom

MONTE CARLO CALCULATION OF THE

ENERGY LOSS SPECTRA FOR GAMMA RAYS

IN SODIUM IODIDE AND CESIUM IODIDE

by

W.F. Miller and William J. Snow 


\section{DISCLAIMER}

This report was prepared as an account of work sponsored by an agency of the United States Government. Neither the United States Government nor any agency Thereof, nor any of their employees, makes any warranty, express or implied, or assumes any legal liability or responsibility for the accuracy, completeness, or usefulness of any information, apparatus, product, or process disclosed, or represents that its use would not infringe privately owned rights. Reference herein to any specific commercial product, process, or service by trade name, trademark, manufacturer, or otherwise does not necessarily constitute or imply its endorsement, recommendation, or favoring by the United States Government or any agency thereof. The views and opinions of authors expressed herein do not necessarily state or reflect those of the United States Government or any agency thereof. 


\section{DISCLAIMER}

Portions of this document may be illegible in electronic image products. Images are produced from the best available original document. 


\section{LEGAL NOTICE}

This report was prepared as an account of Government sponsored work. Neither the United States, nor the Commission, nor any person acting on behalf of the Commission:

A. Makes any warranty or representation, expressed or implied, with respect to the accuracy, completeness, or usefulness of the information contained in this report, or that the use of any information, apparatus, method, or process disclosed in this report may not infringe privately owned rights; or

B. Assumes any liabilities with respect to the use of, or for damages resulting from the use of any information, apparatus, method, or process disclosed in this report.

As used in the above, "person acting on behalf of the Commission" includes any employee or contractor of the Commission, or employee of such contractor, to the extent that such employee or contractor of the Commission, or employee of such contractor prepares, disseminates, or provides access to, any information pursuant to his employment or contract with the Commission, or his employment with such contractor.

Price $\$ 2,00$. Available from the Office of Technical Services, Department of Commerce, Washington 25, D.C. 
ANL-6318

Physics

(TID-4500, 16th Ed.)

AEC Research and

Development Report

ARGONNE NATIONAL LABORATORY

9700 South Cass Avenue

Argonne, Illinois

MONTE CARLO CALCULATION OF THE ENERGY

LOSS SPECTRA FOR GAMMA RAYS IN SODIUM

IODIDE AND CESIUM IODIDE

by

W. F. Miller and William J. Snow

February 1961

Operated by The University of Chicago

under

Contract W-31-109-eng-38 
TABLE OF CONTENTS

Page

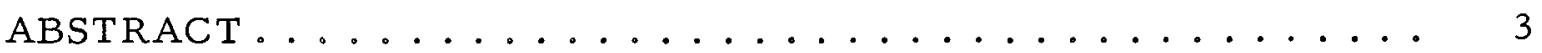

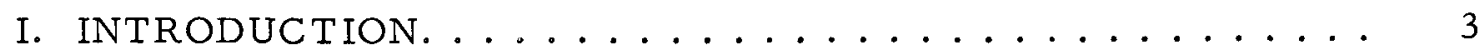

II. PHYSICAL PROCESSES SIMULATED. ............ 5

III. CROSS SECTIONS EMPLOYED ................ 6

IV. PHOTON HISTORIES. ................. 7

A. Photoelectric Event..................... 10

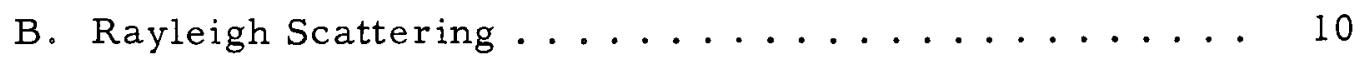

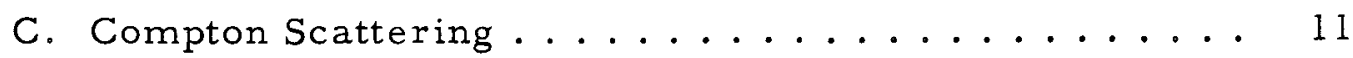

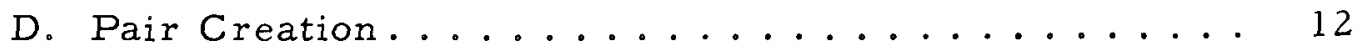

E. Slowing Down of Electrons and Positrons........ 12

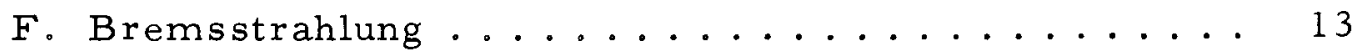

V. COMPARISON WITH EXPERIMENTAL AND

THEORETICAL RESULTS . . . . . . . . . . . . . 14

VI. COMPARISON OF NaI AND CSI RESPONSE SPECTRA . . . . 19

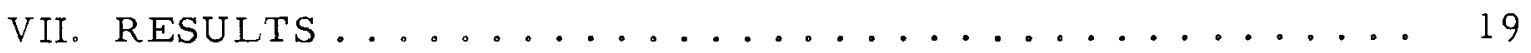

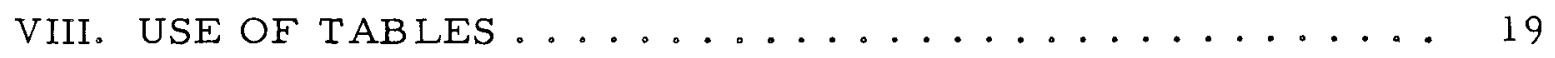

A. TABLE I (Point source on surface of NaI crystal) ... 22

B. TABLE II (Broad parallel beam incident on

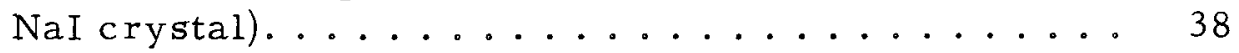

C. TABLE III (Collimated beam incident on NaI crystal beam radius is 1.693 inches) ............. 50

D. TABLE IV (Collimated beam incident on NaI crystal beam radius is 0.25 inch) ................ 57

E. TABLE V (Disc source on crystal axis of

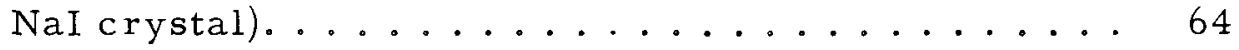

F. TABLE VI (Broad parallel beam incident on sphere. Sphere radius is 1 inch)............... 73

G. TABLE VII (Point source on axis of CsI crystal) . . . 76 


\title{
MONTE CARLO CALCULATION OF THE ENERGY LOSS SPECTRA FOR GAMMA RAYS IN SODIUM IODIDE AND CESIUM IODIDE
}

\author{
by
}

W. F. Miller and William J. Snow

\begin{abstract}
The energy loss spectra for gamma rays in $\mathrm{NaI}$ and CsI crystals have been calculated by the Monte Carlo method. The calculations have been carried out for point sources, broad and collimated parallel beams, and disc sources of mono-energetic gamma rays varying in energy from 0.142 to $14.0 \mathrm{Mev}$. The crystals considered were right circular cylinders, varying from small crystals of height and diameter of 0.5 inch to large crystals of height 12 inches and diameter 9 inches, and in addition for a sphere of 1 -inch radius. Also calculated simultaneously with the energy loss spectrawere the efficiencies and total absorption fractions. This report also includes results which were reported by the authors in the Review of Scientific Instruments, 31, 39 (1960) and 31,861 (1960).
\end{abstract}

\section{INTRODUCTION}

The widespread application of scintillation counter techniques in gamma-ray spectroscopy has motivated theoretical calculations that predict the properties of gamma rays in various materials for use in the design of counter systems as well as in the analysis of complex spectra. Calculations of energy loss spectra have been carried out for $\mathrm{NaI}$ and CsI crystals, 1,2 in the form of right circular cylinders and spheres. The use of CsI as a gamma-ray scintillator has attracted considerable attention because its high stopping power, as compared with that of NaI, produces a slightly larger photofraction for crystals of equal size. These properties are useful in the design of gamma-ray detecting systems in rockets and satellites ${ }^{3}$ where crystal size is a primary consideration.

${ }^{1}$ C. C. Harris et al., Nucleonics 14, 102 (November 1956).

${ }^{2}$ C. T. Schmidt, CsI as a Gamma-ray Spectrometer, Seventh Scintillation Counter Symposium, February 1960, Washington, D.C.

${ }^{3}$ C. E. McIlwain, Scintillation Counters in Rockets and Satellites, Seventh Scintillation Counter Symposium, February 1960, Washington, D.C. 
The computer program constructed for this investigation yields the energy loss spectrum in the form of a histogram of the number of gamma rays experiencing an energy loss in a given energy interval, that is, the energy range is divided into intervals by the set of energy points $\epsilon_{0}, \epsilon_{1}, \ldots, \epsilon_{\mathrm{T}}$, where $\epsilon_{0}=0, \epsilon_{\mathrm{T}}=E_{0}$ (where $E_{0}$ is the energy of the incident gamma ray), and the calculations determine $I_{i}$, the number of gammas that lose, in the crystal, an amount of energy in the interval $\left(\epsilon_{i-1}, \epsilon_{i}\right)$. This histogram is recorded. In all calculations considered herein the energy intervals selected were of equal size and 128 in number.

Let $\mathrm{N}$ be the total number of gamma rays emitted by the source into the hemisphere including the crystal, $G$ be the fraction of these which strike the crystal face, $I_{0}$ be the number of gammas which pass through the crystal without interacting, and $I\left(=\sum_{i=1}^{T} I_{i}\right)$ be the number of interacting gammas. Then

$$
N G=I_{0}+I \text {. }
$$

For a broad parallel beam $G$ is unity; for a point source on the crystal axis $G$ is $1 / 2 \pi$ times the solid angle subtended by the crystal face; and for a disc source $G$ is $1 / 2 \pi$ times the mean solid angle subtended by the crystal face for point sources uniformly distributed across the disc. The quantity herein called the interaction ratio is given by

$$
F=\left[I /\left(I+I_{0}\right)\right],
$$

and the absolute detection efficiency would be

$$
\mathrm{GF}=(\mathrm{I} / \mathrm{N})
$$

In the present investigation the quantities which are calculated for a given crystal, initial energy, and given value of $N$, are $G, I_{0}$, and $I_{i}$ for $i=1,2, \ldots, 128$. The set of $I_{i}$ gives the energy loss spectrum itself and from the other quantities the efficiency and interaction ratio may be determined. The calculations, which employed the Monte Carlo method, were carried out on one of Argonne's digital computers, GEORGE. 4

The computer program is set up to permit calculations for right circular cylinders and spheres of $\mathrm{NaI}$ and $\mathrm{CsI}$ of various sizes, and for initial gamma-ray energies from 0.01 to $15 \mathrm{Mev}$. The types of sources which may be employed are (1) broad parallel beam, (2) narrowly collimated parallel beam, (3) point source on the crystal axis, (4) collimated

$4_{\mathrm{J}}$. Assoc. Computing Machinery $\underline{3}, 45$ (1956). 
point source on the crystal axis, and (5) disc source with the center of the disc on the crystal axis where the disc radius may be any size up to the radius of the crystal. Figure 1 shows schematically the different types of sources.
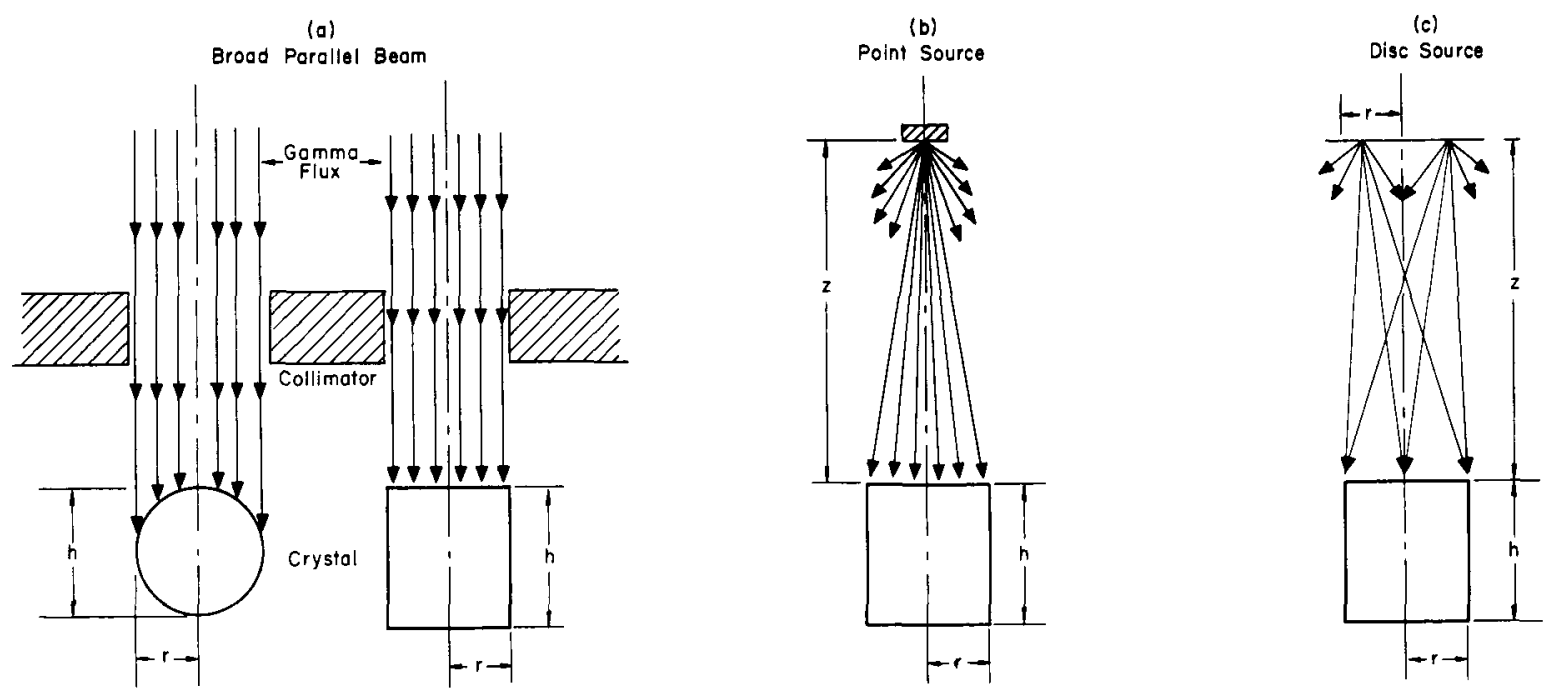

Fig. 1. Diagrams showing source geometries

The present program permits calculations at higher energies than the earlier program, 5 introduces a treatment of bremsstrahlung, and yields the detailed energy loss spectrum which the earlier program did not give.

\section{PHYSICAL PROCESSES SIMULATED}

The primary processes which gamma rays undergo are Rayleigh and Compton scattering, the photoelectric effect, and pair production. During these processes secondary gammas and secondary electrons and positrons are generated. Moreover, in the slowing down of electrons and positrons, bremsstrahlung photons are produced, and photons are created in the annihilation of positrons. The secondary photons considered are the scattered gammas in Rayleigh and Compton scattering, the fluorescent radiation subsequent to the photoelectric effect, gammas created in the annihilation of positrons subsequent to pair production, and bremsstrahlung

${ }^{5}$ W. F. Miller, J. Reynolds, and William J. Snow, Efficiencies and Photofractions for Gamma Radiation on Sodium Iodide Crystals, ANL-5902 (1958). 
photons. The charged particles which must be accounted for are: Compton scattered electrons, photoelectrons, and pair-produced electrons and positrons. All other processes have been neglected since they occur with very small frequency at the energies considered herein. 6

\section{CROSS SECTIONS EMPLOYED}

The X-ray attenuation coefficients in sodium iodide for Rayleigh and Compton scattering, for the photoelectric effect, and for pair production were taken from the work of Grodstein. 7 The coefficients were separated into energy intervals. For each interval the corresponding values, taken from the above-mentioned report, were plotted on very large graph paper with fine mesh; on each of these plots a smooth curve was drawn through the points relevant to a particular interval. Each curve was then approximated by a series of straight lines whose lengths were determined by the shape of the curves. The coordinates of the end points of the line segments (energy, cross section) were stored in the computer. To obtain the cross section for any given energy, the computation consisted of a linear interpolation between the appropriate energy values in the stored table. The cross sections for cesium iodide, which were handled in a similar manner, were taken from the work of Storm, Gilbert, and Israel. 8 The angular distributions for the scattered radiations and the slowing down of the charged particles employ the same formula and algorithms as for sodium iodide except for simple changes of numerical constants, such as binding energies and radiation lengths, which depend on the material under consideration.

No bremsstrahlung spectra for cesium iodide are known to the authors. The sodium iodide bremsstrahlung ${ }^{9}$ spectra were used in this calculation as approximations to the cesium iodide bremsstrahlung spectra. Such approximations are adequate for the energies considered herein, since at these energies the correction for the escape of bremsstrahlung photons affects the area under the photopeak by only a few per cent.

${ }^{6}$ Fano, Spencer, and Berger, Penetration and Diffusion of X Rays, Encyclopedia of Physics (Springer-Verlag, Berlin, Germany, 1958), Vol. 38 II.

${ }^{7}$ Gladys White Grodstein, X-ray Attenuation Coefficients from $10 \mathrm{kev}$ to $100 \mathrm{Mev}$, NBS Circ. No. 583 (April 1957).

8 E. Storm, E. Gilbert, and H. Is rael, Gamma-ray absorption coefficients for elements 1 through 100 derived from the theoretical values of the National Bureau of Standards, LA-2237.

${ }^{9}$ C. D. Zerby and H. S. Moran, Bremsstrahlung spectra in NaI and Air, ORNL-2454 (1958). 


\section{PHOTON HISTORIES}

The complete history of an incident gamma ray and all subsequent radiation is simulated by the Monte Carlo process. For each incident gamma ray, the total amount of energy absorbed by the crystal as a result of the various processes considered is recorded, and a count is placed in the appropriate channel of the energy-loss histogram.

The simulation process proceeds in the following way. The energy of the initial gamma ray is determined by the source energy, and its direction is determined by random sampling from the appropriate source distribution. The initial distribution for the five sources considered in this calculation was handled as follows: 1) For the broad parallel beam it was assumed that the rays were uniformly distributed through the beam and that every ray in the beam would strike the crystal face. The point of impact of the incident gamma was determined by selecting a random point on the face of the crystal from a uniform distribution. The momentum of the gamma is normal to the crystal surface at that point. 2) For the narrowly collimated beam the incident gamma was sampled in the same manner as the broad parallel beam except that the point of impact on the crystal face was restricted to an area smaller than the area of the entire crystal face. 3) For the point source, the direction of emission was randomly chosen from vectors uniformly distributed in the solid angle subtended by the crystal face. The point at which the gamma ray enters the crystal face is determined using the direction cosines of the vector and the distance separating the point source and the crystal face. The fraction of the solid angle $2 \pi$ which the crystal face subtends is equal to the factor $G$ in equation (4). 4) For the disc source the direction of emission was randomly chosen from vectors uniformly distributed in a solid angle that included the entire crystal face. The cone sampled was a right circular cone with apex angle

$$
2 \arctan (2 r / z) \text {. }
$$

Many of the rays emitted in this solid angle do not strike the crystal face; however, many rays can be sampled in an extremely short time and the fraction of these that strike the crystal face can be easily computed. The product of this fraction with the ratio of this solid angle to the solid angle $2 \pi$ is the factor $G$ in equation (5).

For the broad or narrowly collimated parallel beam, $G=1$; for the point source $G$ is calculated from

$$
\frac{1}{2 \pi} \int_{0}^{\tan ^{-1}(r / z)} \sin \theta d \theta \int_{0}^{2 \pi} d \phi=G_{\text {point }} \quad(\text { for } z>0)
$$


and for the disc source

$$
\frac{H}{2 \pi} \int_{0}^{\tan ^{-1}(2 r / z)} \sin \theta d \theta \int_{0}^{2 \pi} d \phi=G_{\text {disc }} \quad(\text { for } z>0),
$$

where $\mathrm{H}$ is the fraction of those gammas emitted in the designated cone which strike the crystal face.

We shall designate the total number of photons emitted as $N, 10$ the number that strike the crystal face as NG, the number that experience at least one interaction as $\mathrm{I}$, and the number that are completely absorbed including all secondary radiation as $A$. Then the interaction ratio, $F$, is given by $I / N G$, and the photofraction, $P$, is given by $A / I$.

Having determined the direction and the point of impact of the incident gamma from the selected source distribution, the position of the first interaction within the crystal is determined by random sampling of the exponential distribution using the total attenuation coefficient:

$$
\mathrm{x}=-1 / \mu \ln \mathrm{R},
$$

where $\mathrm{R}$ is a random number uniform on the interval $(0,1)$ and $\mu$ is the total X-ray attenuation coefficient (see Fig. 2).

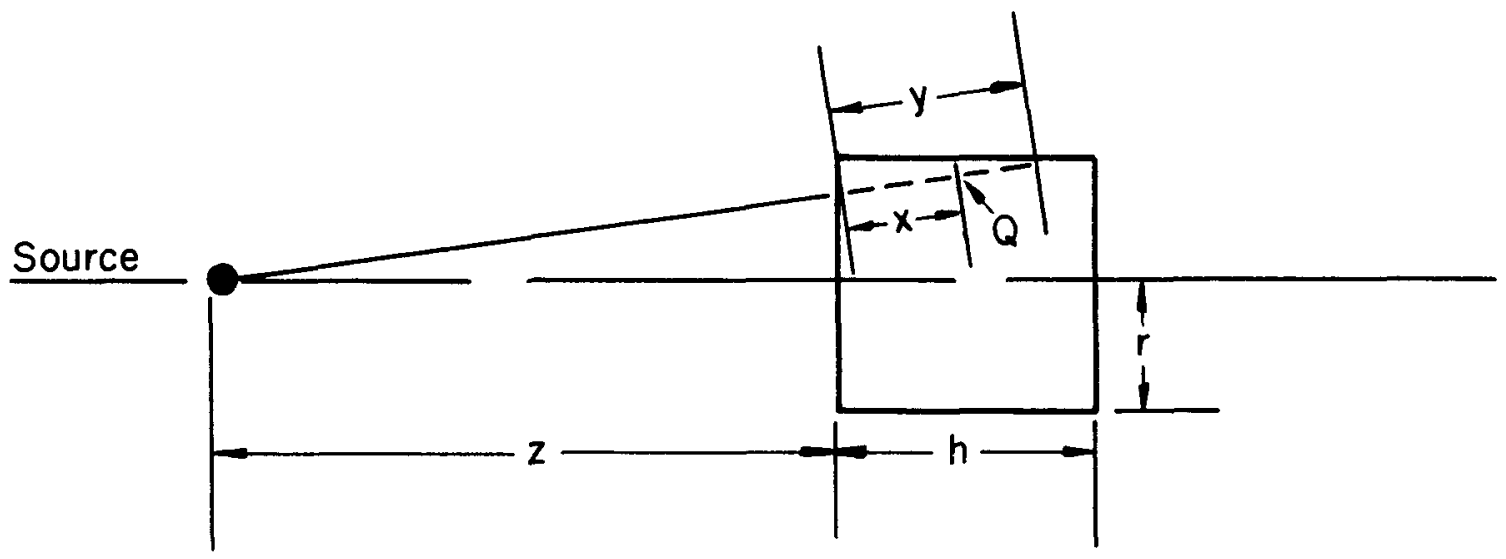

Fig. 2. Path length sampling

$10_{\mathrm{N}}$ and the related quantities represent numbers of gammas in some finite time interval. 
If $\mathrm{x}$ exceeds the length of the ray in the crystal, then the photon has escaped without interaction; a count is made in the $P$ counter and that history is terminated. For those photons not escaping, the position $Q$ of the first collision is determined by the path length selected. The nature of the first collision is determined by sampling the relative distribution of allowable events. Having selected a type of event, the direction of scattering of the particles and their associated energy is determined by sampling the appropriate distributions, which are defined below. The history of all products of a collision are traced through the crystal until all the energy of the incident ray has escaped or been absorbed by the crystal. Figure 3 is a schematic diagram showing this calculation.

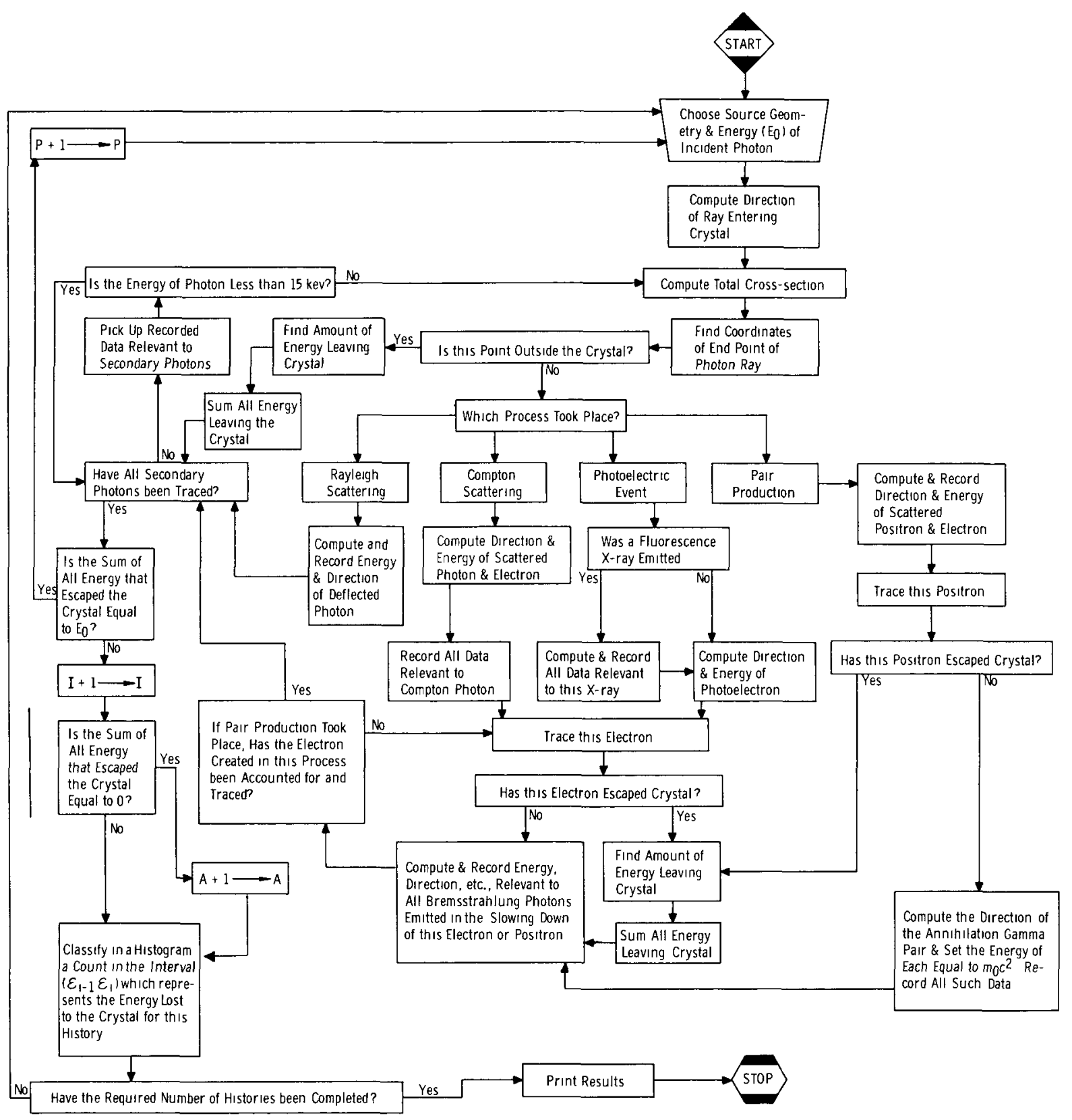

Fig. 3. Schematic Flow Diagram of Energy Loss Spectrum Calculation 


\section{A. Photoelectric Event}

The two secondary radiations that occur in a photoelectric collision are the photoelectron and the fluorescent $\mathrm{X}$ rays. For unpolarized gamma rays, the azimuthal angle for the ejected photoelectron is uniformly dis tributed, and the polar angle is distributed according to

$$
\operatorname{dn} \sim \sin ^{2} \theta\left\{1+2 \beta\left[1+\frac{E_{B}}{E_{e}}\right] \cos \theta\right\} \sin \theta d \theta,
$$

where $E_{B}$ is the shell binding energy (herein taken as the $\mathrm{K}$ shell binding energy for iodide), $E_{e}$ is the energy of the photoelectron, $\beta$ is the ratio of the electron velocity to the velocity of light, and $\theta$ is the polar angle of scattering for the photoelectron. ${ }^{11}$ If $E_{0}$ is the energy of the incident gamma, then

$$
E_{e}=E_{0}-E_{B} .
$$

The fluorescent $X$-ray yield for sodium iodide is about 83 per cent. The energy of the fluorescent $\mathrm{X}$ ray is taken as the energy of the $\mathrm{K}_{\alpha} \mathrm{X}$ ray for iodide, and the $\mathrm{X}$ ray is emitted isotropically.

\section{B. Rayleigh Scattering}

In Rayleigh scattering all the recoil momentum is absorbed by the atom as a whole; thus there is no scattered electron to account for. Furthermore, due to momentum considerations, the atom can absorb very little energy from the photon. In the present work the atom was considered to absorb a very small amount of energy (less than l kev) and the photon was deflected through a small angle. Since Rayleigh scattering is important only at low energies, the following approximations are introduced for the angular distribution of Rayleigh scattered photons. An examination of the calculations of Moon 12 show that most of the scattering is confined to angles less than the so-called critical angle, $\theta_{c}$, defined by

$$
\theta_{c}=2 \sin ^{-1}\left(0.026 \mathrm{z}^{1 / 3} \frac{\mathrm{m}_{0} \mathrm{c}^{2}}{\mathrm{h \nu}}\right) \text {. }
$$

${ }^{11}$ A. H. Compton and S. K. Allison, X Rays in Theory and Experiment,

D. Van Nostrand Co., Inc., Princeton, N. J. (1935), Second Edition, p. 579.

12 P. B. Moon, Proc. Phys. Soc. (London) A63, 1189 (1950). 
Moreover, the angular distribution does not deviate far from linearity when the logarithm of the angular distribution is plotted against the cosine of the angle of scattering up to $\theta_{\mathrm{c}}$. Although Moon's calculations are not for $\mathrm{NaI}$, the relative behavior does not depend strongly on $\mathrm{Z}$. Therefore, the angular distribution will be crudely given by $\mathrm{dn} \sim \mathrm{e}^{-\mathrm{b}} \mu \mathrm{d} \mu$ where

$$
\mu=\cos \theta \text { and } b \text { is a constant. }
$$

This distribution was normalized and the samples were taken between $\theta=0$ and $\theta=\theta_{c}$.

C. Compton Scattering

In a compton collision, the scattering of an incident energetic photon by an atomic electron that is initially at rest can be described as a two-particle collision if we neglect the small effects due to the binding of the electron to the nucleus. The energy and momentum of the incident photon will be changed in the collision process, yielding energies, for the scattered photon and the recoil electron for each angle of scattering, determined by the conservation laws. For unpolarized radiation, the polar angle $\theta$ of scattering of the scattered photon is determined by random sampling of the Klein-Nishina distribution, given by

$$
\mathrm{d} \phi=\frac{\mathrm{r}_{0}^{2}\left(1+\cos ^{2} \theta\right)}{2\left[1+\frac{E_{0}}{m_{0} c^{2}}(1-\cos \theta)\right]^{2}}\left\{1+\frac{\left(E_{0} / m_{0} c^{2}\right)^{2}(1-\cos \theta)^{2}}{\left(1+\cos ^{2} \theta\right)\left[1+\frac{E_{0}}{m_{0} c^{2}}(1-\cos \theta)\right]}\right\} d \Omega,
$$

where $r_{0}$ is the classical electron radius and $d \phi$ is the differential cross section for the scattering process in which the incident photon of energy $E_{0}$ is scattered by a free electron through the angle $\theta$ into the solid angle $\mathrm{d} \Omega$ with energy $E^{\prime}$; the azimuthal angle is sampled from the uniform distribution. Having determined the energy and direction of the scattered photon, the energy of the recoil electron is simply $E_{e}=E_{0}-E^{\prime}$, and its polar angle of scattering, $\theta^{\prime}$, determined from the conservation laws is given by

$$
\tan \theta^{\prime}=\frac{E^{\prime} \sin \theta}{E-E^{\prime} \cos \theta},
$$

and again the azimuthal angle of the electron is sampled from the uniform distribution. One then carries out the transformation from the coordinate system of the gamma to the crystal coordinate system to obtain the directions of the scattered photon and electron. 


\section{Pair Creation}

A photon with energy greater than $2 \mathrm{~m}_{0} \mathrm{c}^{2}$ in the vicinity of the nucleus can create a positron-electron pair. In the event this process is determined, the mean case is considered wherein the kinetic energy over and above $2 \mathrm{~m}_{0} \mathrm{c}^{2}$ is shared equally by the electron and positron, that is,

$$
E^{+}=E^{-}=\frac{1}{2}\left(E_{0}-2 m_{0} c^{2}\right)
$$

Furthermore, it is assumed that the polar angle of scattering is the average angle between the incident photon and the direction of motion of the electron or positron, given by 13

$$
\bar{\theta}_{ \pm} \cong \mathrm{m}_{0} \mathrm{c}^{2} / \mathrm{E}^{ \pm}
$$

The azimuthal angles for the electron and positron are sampled from the uniform distribution, but differ by $180^{\circ}$.

In the treatment of annihilation radiation subsequent to positron creation, wherein the positron colliding with an atom creates a vacant state into which an atomic electron may fall releasing energy $2 \mathrm{~m}_{0} \mathrm{c}^{2}$, the energy may be emitted in the form of a gamma pair, each with energy $\mathrm{m}_{0} \mathrm{c}^{2}$, or may be transferred to a second electron which leaves the atom. For the present calculation the former was chosen due to the extremely low probability of occurrence of the later. It is assumed that the positrons are completely stopped before annihilation. If the positron escapes the crystal, then no annihilation takes place. If, however, the positron is stopped in the crystal, annihilation takes place and the annihilation gamma pair is emitted isotropically from the point of annihilation, but oppositively directed.

E. Slowing Down of Electrons and Positrons

The slowing down of Compton scattered electrons, photoelectrons, and pair-produced electrons and positrons is handled as follows. The process of slowing down of charged particles involves very large numbers of interactions with relatively small energy loss per interaction, in contrast to the slowing down of gamma rays, which undergo rather few large energy-loss interactions. Because of the large number of interactions it is too time consuming in the calculations to treat electron and positron slowing down by the Monte Carlo method, and, in fact, only a few of the events associated with the slowing down are of interest to us. Herein,

$13 \mathrm{H}$. A. Bethe and J. Ashkin, Passage of Radiations through Matter, Experimental Nuclear Physics, John Wiley and Sons, Inc., New York (1953), Vol.I. 
the approximate theory of Wilson 14 is used to determine the mean path length and energy loss of the charged particle. It is assumed that the electron or positron travels its mean distance, and in a straight line, in the direction determined by the angular distributions discussed above. The mean distance traveled by an electron (or positron), in radiation lengths, is

$$
r=(\ln 2) \ln \left[\frac{E_{0}}{E_{c} \ln 2}+1\right],
$$

where $E_{0}$ is the initial energy of the particle and $E_{C}$ is the so-called critical energy. In this study the values of $E_{C}$ and the radiation lengths were taken from Table 8 of the article by Bethe and Ashkin. 13

If the mean length, $r$, takes the particle outside the crystal, the energy carried away is computed by solving Eq. (15) for $E_{0}$, where $r$ is taken as the distance traveled outside the crystal, designated as $r^{\prime}$. Then the energy carried away from the crystal is

$$
E^{\prime}=\left[\exp \left(r^{\prime} / \ln 2\right)-1\right] E_{C} \ln 2,
$$

and, of course, $\left(E_{0}-E^{\prime}\right)$ is absorbed by the crystal. The Wilson slowing down theory includes excitation and ionization losses and radiation losses in the field of the nucleus, that is, bremsstrahlung. It is assumed that all energy emitted by excitation and ionization is absorbed by the crystal, whereas bremsstrahlung photons will be traced through the crystal just as primary photons.

F. Bremsstrahlung

In the slowing down of electrons and positrons, bremsstrahlung photons are produced, and these must be traced through the crystal. In the relativistic case, most of the radiation is emitted into small angles in the forward directions. The bremsstrahlung spectrum for NaI has been calculated by Zerby and Moran ${ }^{9}$ using the Born approximation. In their report they present curves giving the number of photons per unit energy range against photon energy for a number of initial energies. In the present calculations the energy range was divided up into several intervals, and from the calculated spectrum the mean number of photons were calculated for each interval; their energy was taken to be the mean energy for the interval. For each charged particle the mean number of photons were distributed at equal intervals along the particle track. If the charged particle escapes the crystal, the residual energy is calculated as indicated

14R. R. Wilson, Phys. Rev. 79, 204 (1950). 
above, Sect. E, and the bremsstrahlung is calculated as for an electron with initial energy equal to the energy absorbed by the crystal. The approximation introduced by this procedure has little effect on the overall calculations. All bremsstrahlung photons were assumed to be emitted at the mean angle

$$
\theta_{\mathrm{b}} \cong\left(\mathrm{m}_{0} \mathrm{c}^{2} / \mathrm{E}_{\mathrm{i}}\right)
$$

down to a minimum angle $\theta_{\mathrm{min}}$.

\section{COMPARISON WITH EXPERIMENTAL AND THEORETICAL RESULTS}

Various theoretical response spectra have been compared with a number of experimental spectra. Figures 4,5 and 6 show comparison of the present calculation with the experimental measurements of Heath. 15 Figures 7 and 8 compare with the experimental work of Crouthamel, 16 and Fig. 9 with that of Bollinger and Kennett. 17 In all cases the calculations are scaled to agree with the experimental curves so that the ordinates represent counts per channel. These calculations appear to reproduce the structure of the experimental curves remarkably well.

The theoretical curves coincide everywhere with the experimental except in the low-energy portions, where the experimental ordinates are slightly higher. This is expected because in any experimental arrangement the low-energy portions of the spectra will be increased by backscattering from various sources or bremsstrahlung from accompanying charged particles.

In the present calculation of the energy-loss spectrum, no account was taken of the resolution effects accompanying light collection within the crystal and its amplification through the photomultiplier system. For comparison with these experimental response spectra, the energy-loss spectrum is "smeared out" by application of a Gaussian resolution function with an empirically determined half-width.

${ }^{15}$ R. L. Heath, Scintillation Spectroscopy Gamma-ray Spectrum Catalogue, IDO-16408, Phillips Petroleum Company (July 1, 1957).

${ }^{16}$ C. E. Crouthamel, Applied Gamma-ray Spectrometry, Pergamon Press (1960).

17 T. J. Kennett (private communication). 


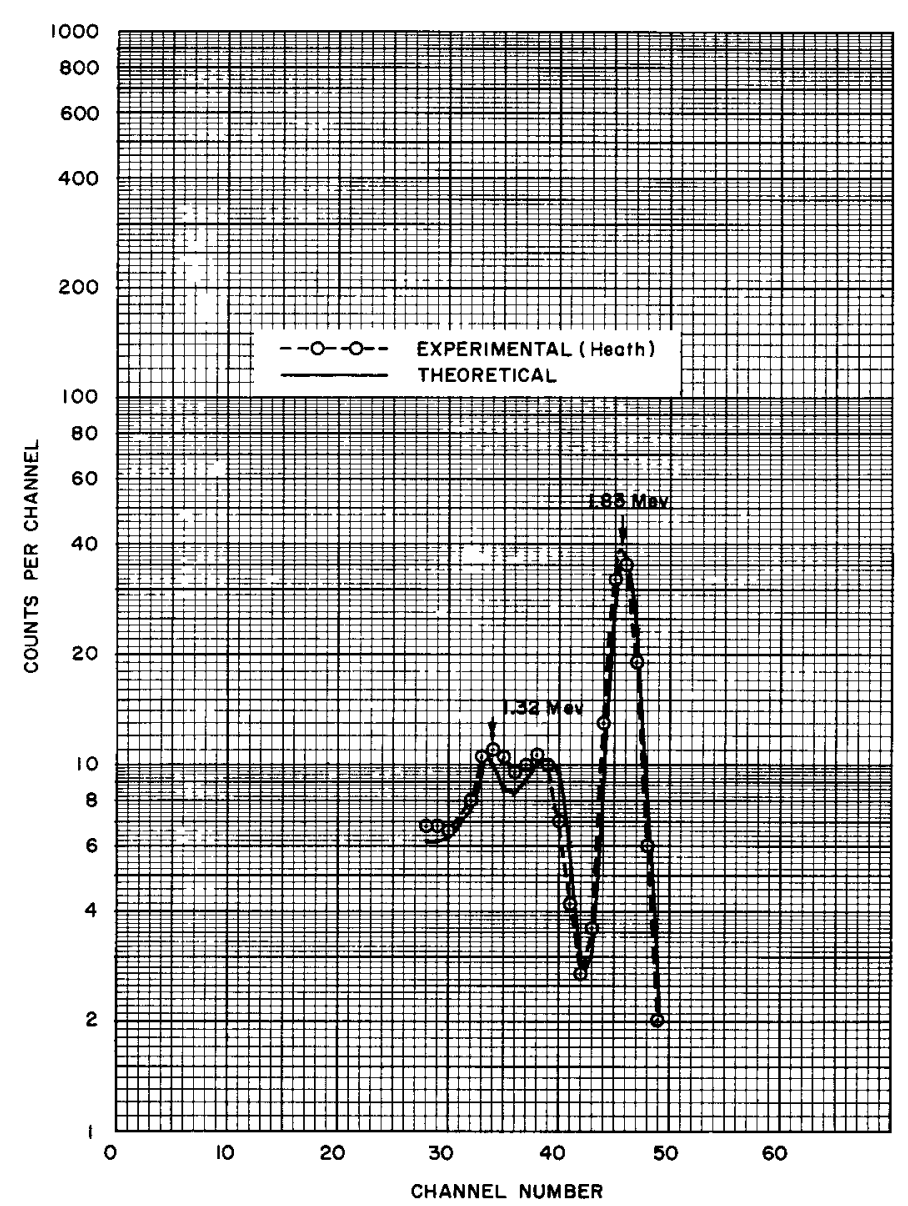

Fig. 4. Response spectrum for a point source incident on a 3 -in. diameter by 3 -in. height $\mathrm{NaI}(\mathrm{T} 1)$

crystal. Source energy is

$1.83 \mathrm{Mev}$ and is located $10 \mathrm{~cm}$ above crystal face on the crystal axis.

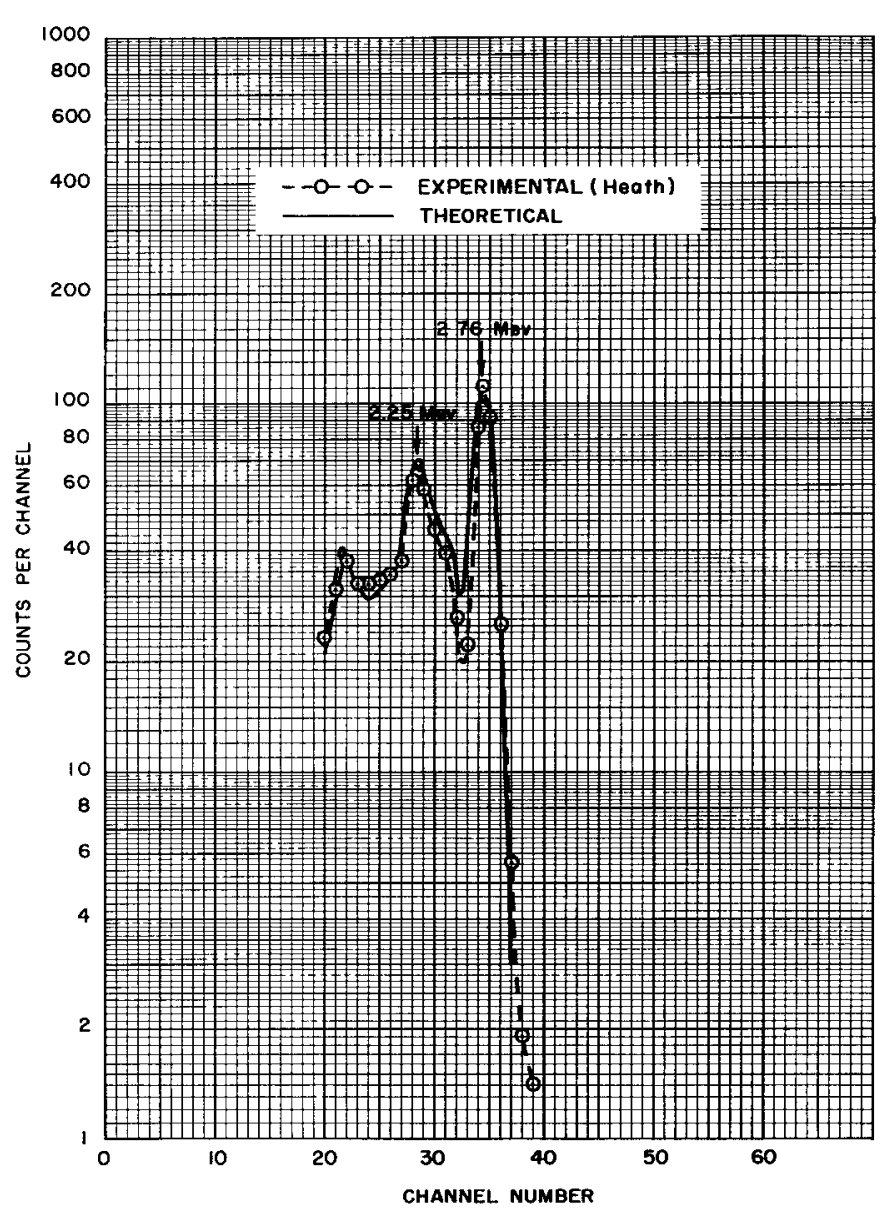

Fig. 5. Response spectrum for a point source incident on a 3-in. diameter by 3 -in. height $\mathrm{NaI}(\mathrm{T} \mathrm{l})$

crystal. Source energy is $2.76 \mathrm{Mev}$ and is located $10 \mathrm{~cm}$ above crystal face on the crystal axis. 


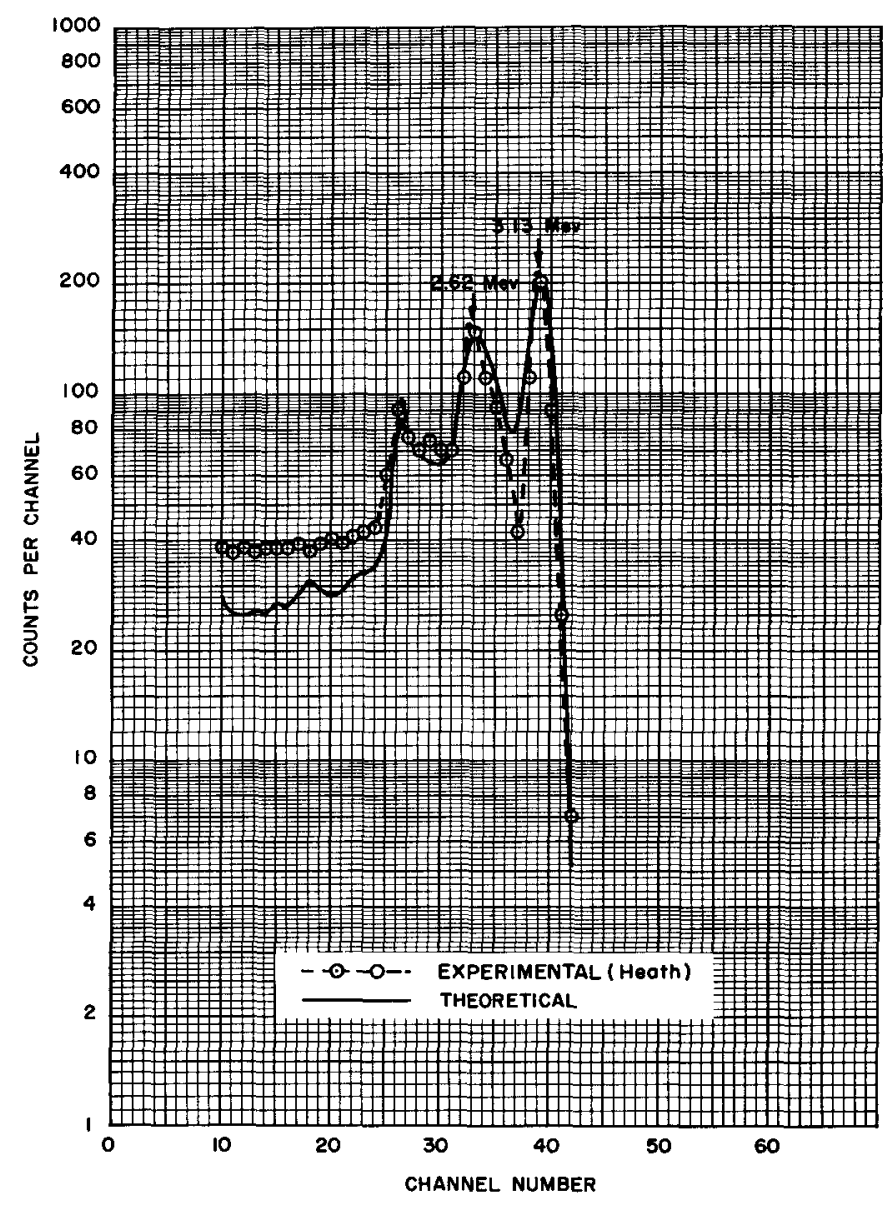

Fig. 6. Response spectrum for a point source incident on a 3-in. diameter by 3 -in. height $\mathrm{NaI}(\mathrm{T} 1)$ crystal. Source energy is $3.13 \mathrm{Mev}$ and is located $10 \mathrm{~cm}$ above crystal face on the crystal axis.

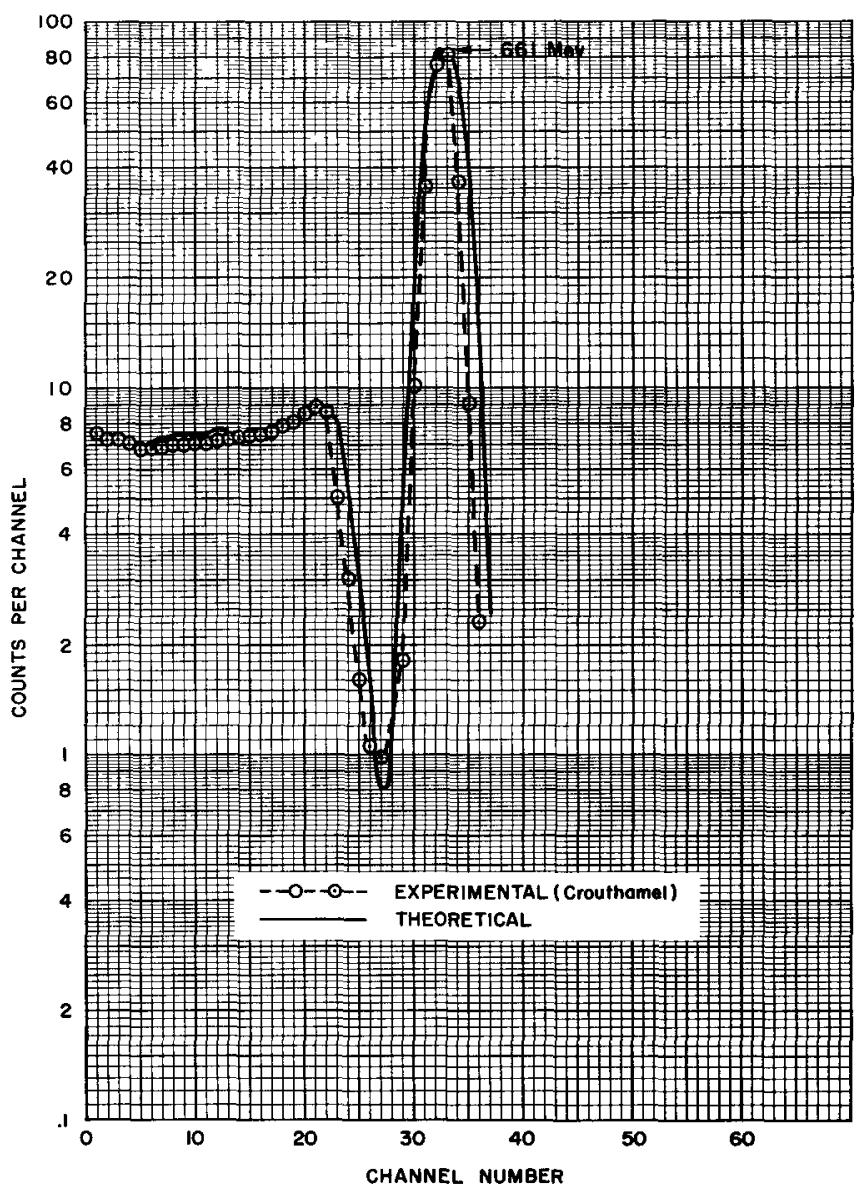

Fig. 7. Response spectrum for a point source incident on a 4-in.diameter by 4 -in. height $\mathrm{NaI}(\mathrm{T} 1)$ crystal. Source energy is $0.661 \mathrm{Mev}$ and is located $5.51 \mathrm{in}$. above crystal face on the crystal axis. 


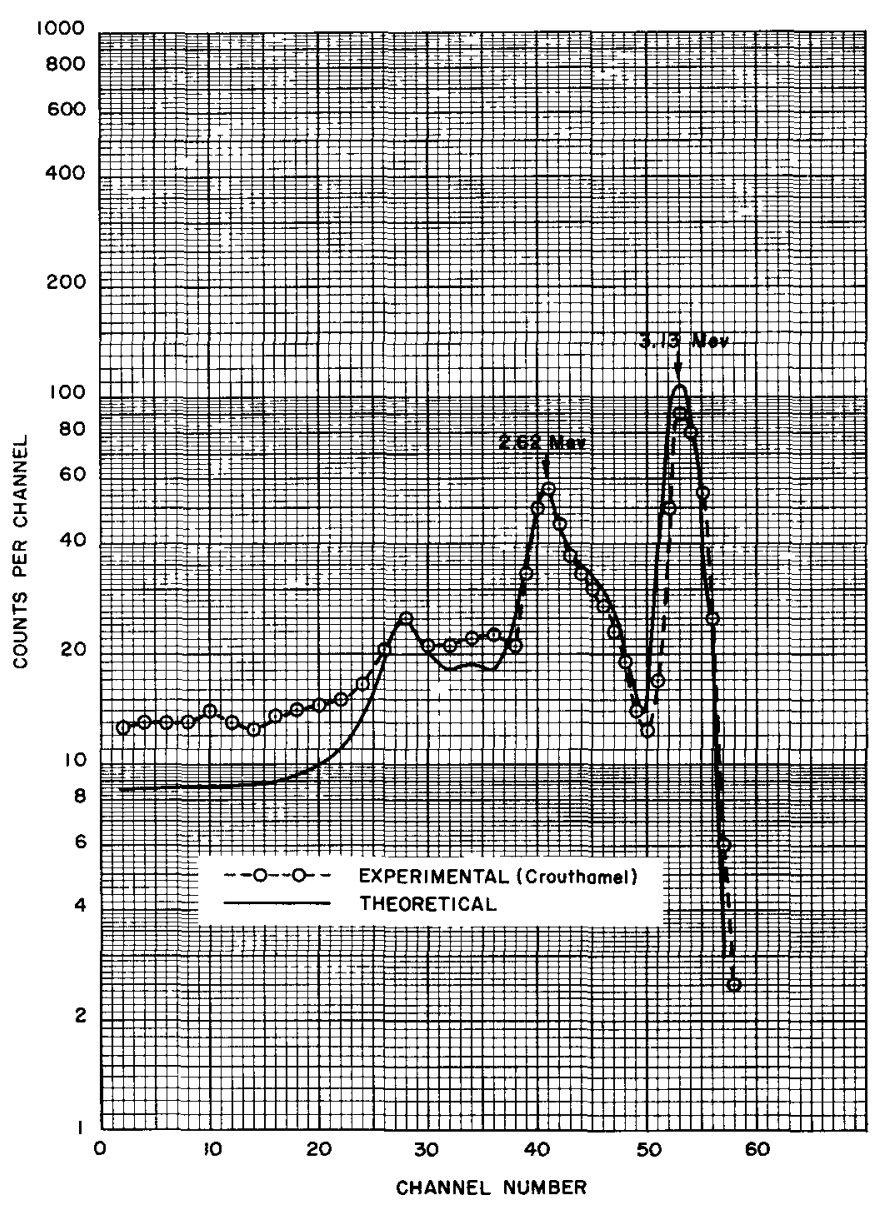

Fig. 8. Response spectrum for a point source incident on a 4-in. diameter by 4-in. height $\mathrm{NaI}(\mathrm{Tl})$ crystal. Source energy is $3.13 \mathrm{Mev}$ and is located $0.39 \mathrm{in}$. above crystal face on the crystal axis.

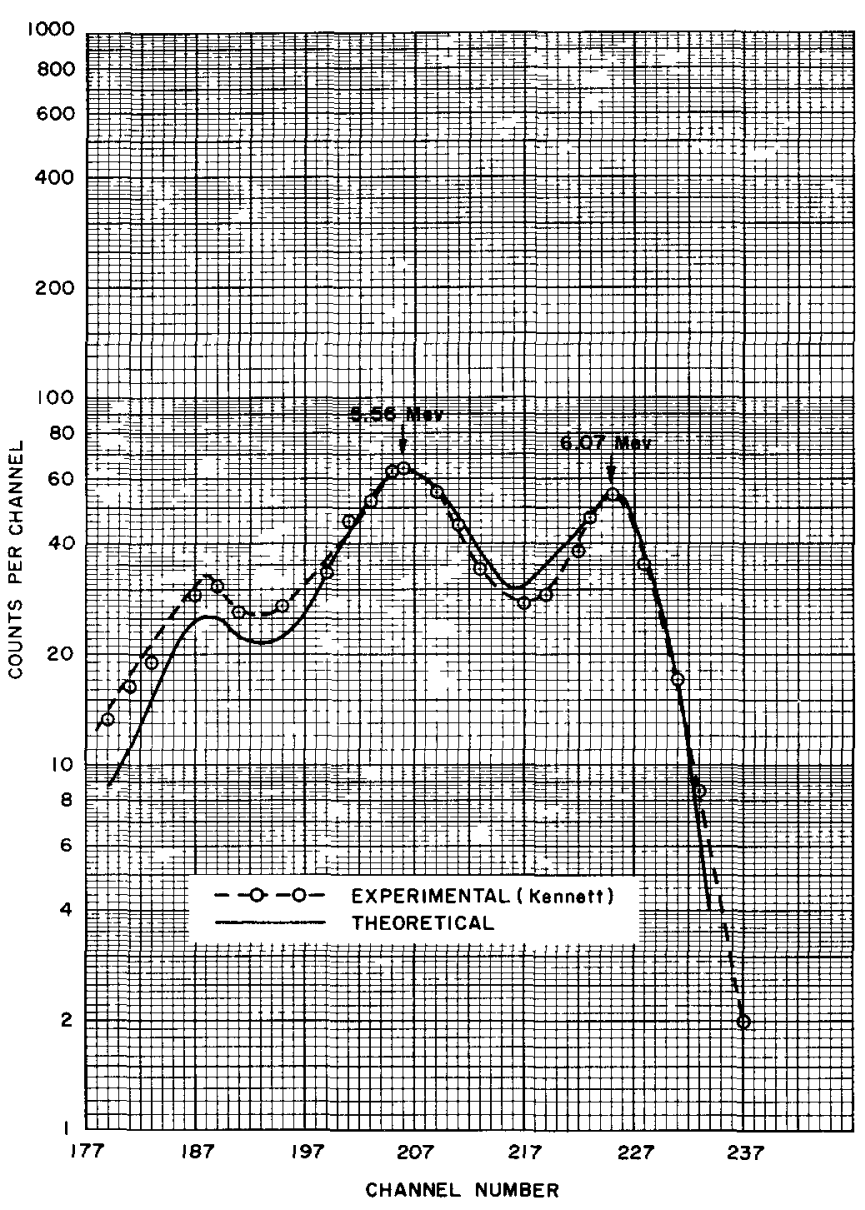

Fig. 9. Response spectrum for a disc source incident on a 4-in. diameter by 4 -in. height $\mathrm{NaI}(\mathrm{Tl})$ crystal. Source energy is 6.07 Mev and the disc is 1ocated 3 in. above crystal face. 
If the continuous energy-loss spectrum for gamma rays of initial energy $E_{0}$ is given by $\mathrm{I}(\epsilon) \mathrm{d} \epsilon$ and the resolution function is

$$
(2 \pi \alpha \epsilon)^{-1 / 2} \exp \left[-\frac{\left(\epsilon-\epsilon^{\prime}\right)^{2}}{2 \alpha \epsilon}\right],
$$

then the experimental response function for gamma rays of initial energy $E_{0}$ is

$$
R\left(\epsilon^{\prime}\right)=\int_{0}^{E_{0}} \frac{1}{(2 \pi \alpha \epsilon)^{1 / 2}} \exp \left[\frac{-\left(\epsilon-\epsilon^{\prime}\right)^{2}}{2 \alpha \epsilon}\right] I(\epsilon) d \epsilon
$$

Since the energy loss spectrum is in the form of a histogram of, $\mathrm{I}\left(\epsilon_{\mathrm{i}}\right)$, the discrete response function $\mathrm{N}\left(\epsilon_{\mathrm{j}}\right)$ is given by

$$
\mathrm{N}\left(\epsilon_{\mathrm{j}}^{\prime}\right)=\sum_{\mathrm{i}=0}^{\mathrm{T}} \frac{\omega_{\mathrm{i}} \exp \left\{-\left[\left(\epsilon_{\mathrm{i}}-\epsilon_{\mathrm{j}}^{\prime}\right)^{2}\right] / 2 \alpha \epsilon_{\mathrm{i}}\right\}}{\left(2 \pi \alpha \epsilon_{\mathrm{i}}\right)^{1 / 2}} \mathrm{I}\left(\epsilon_{\mathrm{i}}\right)
$$

where $\omega_{0}=\omega_{T}=\frac{1}{2}$ and $\omega_{i}=1, i \neq 0$ or $\mathrm{T}$. The constant $\alpha$ is chosen empir ically to give a selected per cent half-width at maximum energy.

Whereas the resolution function (17) is known to agree with experiment at low energies, it deviates from experiment at high energies. For all comparisons with experiment herein, the resolution function (17) is used. 


\section{COMPARISON OF NaI AND CSI RESPONSE SPECTRA}

Figures 10, 11, 12 and 13 show theoretical response spectra for cesium and sodium iodide crystals of the same size.

In these calculations the same resolution function as described above was used. In all cases the $\mathrm{NaI}$ and $\mathrm{CsI}$ response curves presented herein show close agreement with each other. The ordinates represent the actual number of counts resulting from the Monte Carlo calculation.

\section{RESULTS}

Tables I through $\mathrm{V}$ present the gamma-ray energy loss spectra in $\mathrm{NaI}$ for various crystal sizes and source geometries. The crystals are in the form of right circular cylinders. Table VI presents the loss spectrum in a NaI spherical crystal. The source considered herein [see Fig. l(a)] was a broad parallel beam incident on the entire surface of the hemisphere. The radius of the sphere was one inch. Table VII presents the energy-loss spectra in CsI for a point source on the crystal axis for various crystal sizes, the crystals being right circular cylinders. Energy-loss spectra rather than response spectra are presented since one can then apply any resolution function desired to obtain the appropriate response function. The distribution of counts in each channel follows the multinomial distribution so that the standard deviation for each channel is approximately given by the square root of the number of counts.

\section{USE OF TABLES}

In all tables presented herein the quantity I represents the number of gammas which interact at least once; $A$ is the number of gammas that are completely absorbed, and $P$ is the number of gammas which pass through the crystal without interacting. In $\mathrm{T}$ able $\mathrm{V}$ the quantity $\mathrm{T}$ is the number of rays emitted in the cone, subtended by a point on the disc with apex angle $2 \tan ^{-1}(2 \mathrm{r} / \mathrm{z})$, that completely miss the crystal; therefore the total number of rays emitted will be given by

$$
\text { Total }=\mathrm{T}+\mathrm{I}+\mathrm{P} \text {. }
$$

The full energy spread of the gamma quantum is spanned by 128 channels, with the counts in each channel given in a rectangular array; the channels start with the lowest energy channel on the left of the first row, then proceed from left to right across each row. Thus the highest energy channel is on the right of the last row. 


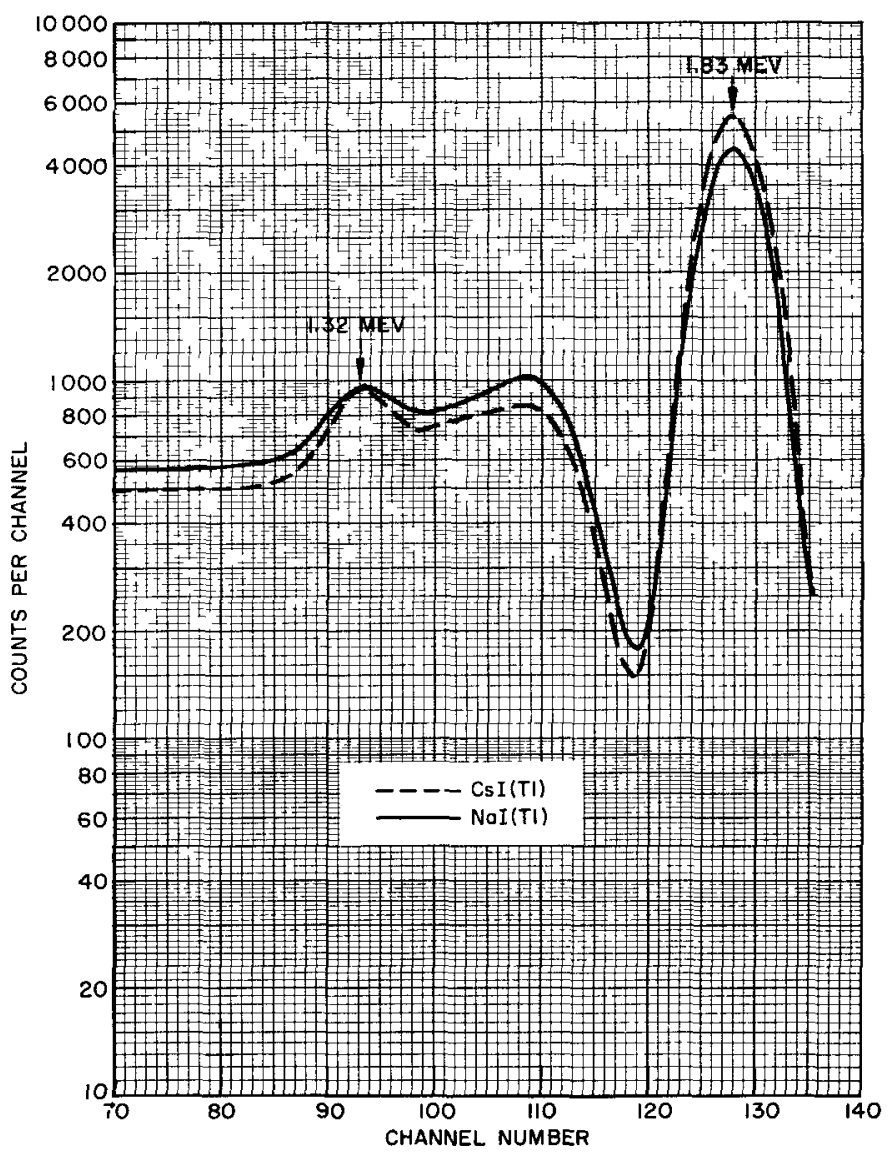

Fig. 10. Response spectra for a point source incident on a 3 -in. diameter by 3 -in. high crystal. Source energy is $1.83 \mathrm{Mev}$ and is located $10 \mathrm{~cm}$ above the crystal face on the crystal axis.

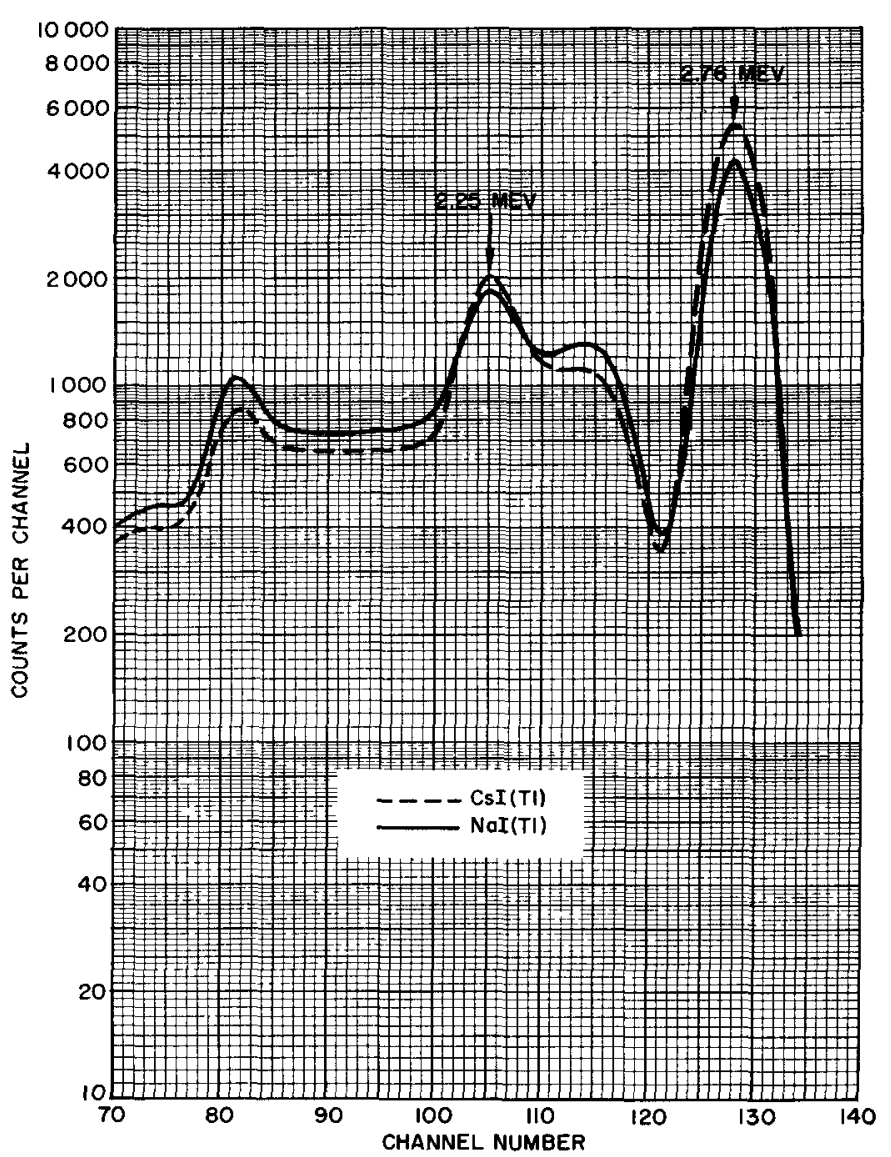

Fig. 11. Response spectra for a point source incident on a 3-in. diameter by 3-in. high crystal. Source energy is $2.76 \mathrm{Mev}$ and is located $10 \mathrm{~cm}$ above the crystal face on the crys tal axis. 


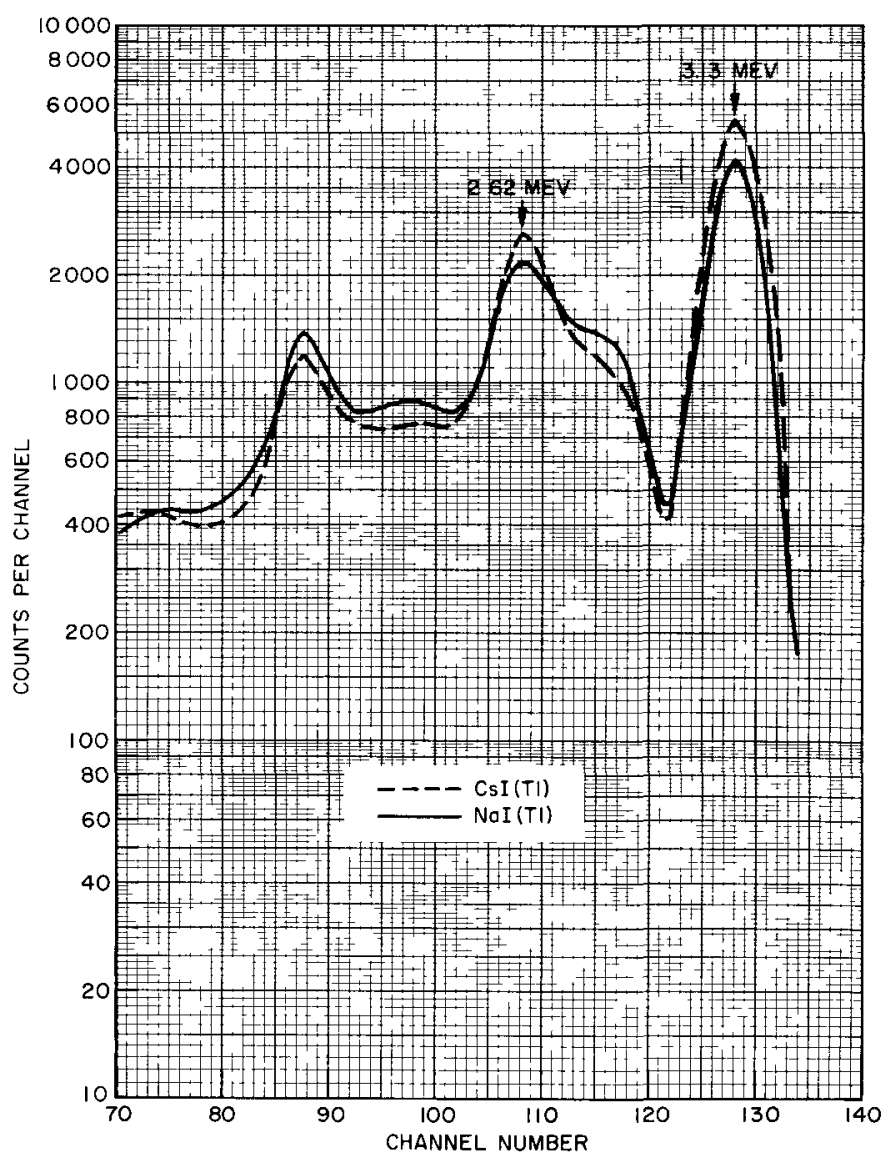

Fig. 12. Response spectra for a point source incident on a 3-1n. diameter by $3-1 \mathrm{n}$. high crystal. Source energy is $3.13 \mathrm{Mev}$ and is located $10 \mathrm{~cm}$ above the crystal face on the crystal axis.

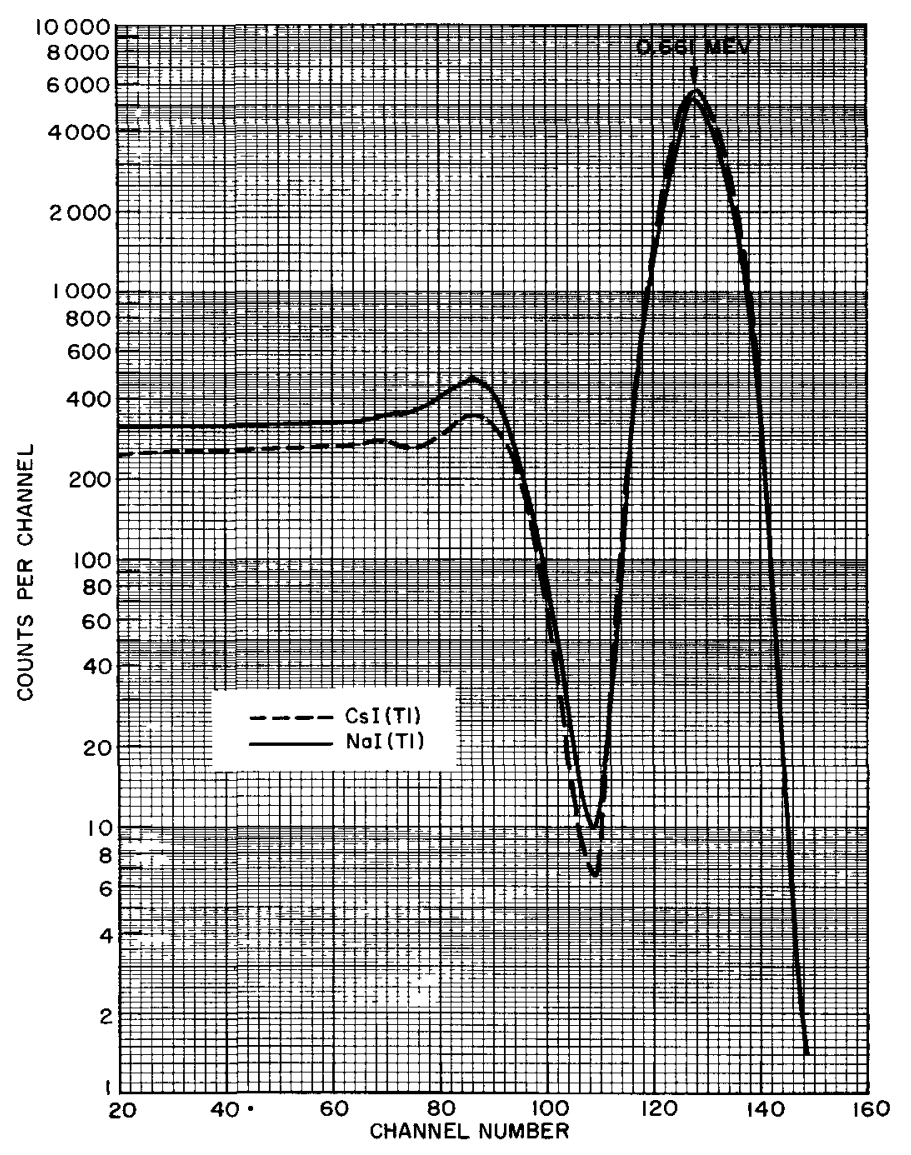

Fig. 13. Response spectra for a point source incident on a 4-in. diameter by 4-in. high crystal. Source energy is $0.661 \mathrm{Mev}$ and is located 5.51 in. above the crystal face on the crystal axis. 
TABLE I

POINT SOURCE ON SURFACE OF NAI CRYSTAL.

CRYSTAL HEIGHT $=01.000000$ IN. CRYSTAL RADIUS $=00.500000 \mathrm{IN}$. $I=10000$

00411000580006400059000610005200053000350004500035000460005800045000400004200044 $0003200045 \quad 0004500050 \quad 00048 \quad 00034 \quad 00028 \quad 00038 \quad 0004200048 \quad 0003500036 \quad 00040 \quad 00036 \quad 00032 \quad 00036$

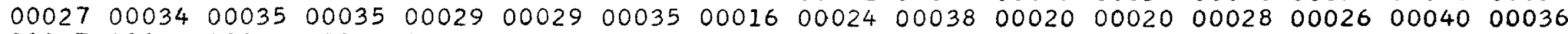

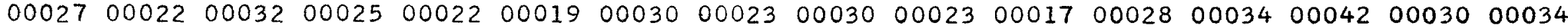
$00030 \quad 000390002700006 \quad 0000400001000020000200004000030000400003000010000200003 \quad 00001$ $00000 \quad 00000 \quad 00000 \quad 0000200000 \quad 00000 \quad 00000 \quad 00000 \quad 00000 \quad 00000 \quad 00000 \quad 0000000000 \quad 0000000000 \quad 00000$ 00000000000000000000000000000000000000000000000000000000000000000000000000000000 $00000000000000000000001560000600000 \quad 000000000000000000000000000000000000000007021$

CRYSTAL HEIGHT $=01.000000$ IN. CRYSTAL RADIUS $=00.500000$ IN. $I=10000$

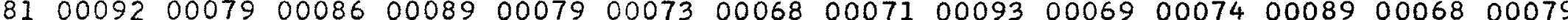
$0006200071 \quad 000750008500071 \quad 00073 \quad 00063000740007200063000550006500066000500005400072$ $000670007600058 \quad 00076 \quad 0007900058 \quad 000640005900075000640007200073000550004700068 \quad 00063$ $000650005900050 \quad 00058000590005500061000690006600059000720005700061000590006500063$ $000610006400050 \quad 0007300066000620006900068000680005500070 \quad 00076 \quad 00071000810006100064$ 00066000710007400072000840007300072000930007800090000880009400041000230001600013 $00011000110001200009000080000800003 \quad 00006 \quad 0000100002000040000000001000010000000000$ $00000 \quad 0000000000 \quad 0000000000 \quad 00000000000000000000000000000000049000000000000000 \quad 03188$

CRYSTAL HEIGHT $=01.000000$ IN. CRYSTAL RADIUS $=00.500000 \mathrm{IN}$. $I=10000$ $A=01859$ INCIDENT ENERGY $=01.170000 \mathrm{MEV}$. SOURCE HEIGHT $=00.000000$ IN. 00165000550007200058000680007900069000610006500063000640006400067000720006400074 $000940005500064000610006200072000740006800071000740006700050 \quad 000730007500056 \quad 00060$ $0006300070 \quad 00070 \quad 00058 \quad 00056 \quad 00067000590005900074000590006200084000620005400066 \quad 00061$ $000610005100063000630006800066 \quad 00066 \quad 00071000660006300066 \quad 0006900072000740006300061$ $\begin{array}{llllllllllllllll}00063 & 00068 & 00046 & 00082 & 00066 & 00059 & 00072 & 00062 & 00088 & 00064 & 00069 & 00085 & 00081 & 00075 & 00070 & 00056\end{array}$ $000880007500076 \quad 00082000770008700079001030008300108000810008700097001040011100101$ $\begin{array}{llllllllllllllllll}0 & 0093 & 00110 & 00111 & 00120 & 00111 & 00111 & 00135 & 00135 & 00145 & 00046 & 00026 & 00025 & 00024 & 00023 & 00012 & 00011\end{array}$ 00005000080000600004000020000300000000000000000001000010000000002000210000001861

INCIDENT ENERGY $=00.279000$ MEV. $\begin{array}{ll}\text { INCIDENT ENERGY } & =00.661000 \text { MEV. } \\ \text { SOURCE HEIGHT } & =00.000000 \text { IN. }\end{array}$ . 
TABLE I (CONTINUED)

CRYSTAL HEIGHT $=01.000000$ IN. CRYSTAL RADIUS $=00.500000 \mathrm{IN}$. $I=10000$

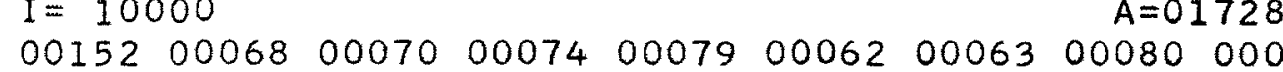
$000610006500066 \quad 00035000550004700080 \quad 0007200074$ 000610006900054000720005400059000610005200063 $\begin{array}{lllllllll}00059 & 00060 & 00057 & 00061 & 00069 & 00066 & 00060 & 00062 & 00071 \\ 00065 & 00075 & 00046 & 00064 & 00072 & 00061 & 00057 & 00067 & 00064\end{array}$ 000720007700087000710007800081000750008700087 001050009600103001030008800131001 000120001400005000060000200003

$\begin{array}{lllllllllll}0 & 075 & 00087 & 00087 & 00096 & 00081 & 00087 & 00080 & 00086 & 00121 & 00118\end{array}$ $\begin{array}{llllllllll}0 & 000123 & 00135 & 00148 & 00081 & 00038 & 00024 & 00022 & 00022\end{array}$

CRYSTAL HEIGHT $=01.000000$ IN. CRYSTAL RADIUS $=00.500000$ IN. $I=10000$

$000850005500056 \quad 0004700043 \quad 00065000670005500058$ $000720006900050 \quad 0006700058 \quad 0006200059000610004700046 \quad 0004400060000570005700058 \quad 00052$ 00069000550006700059000860005200054 $0005800056 \quad 00056 \quad 000660006700056 \quad 00057000770005400064000570006200063000580005100044$ $000490006200058 \quad 00063 \quad 00050 \quad 000650004500051 \quad 0006300056 \quad 000600004700051000450005500056$

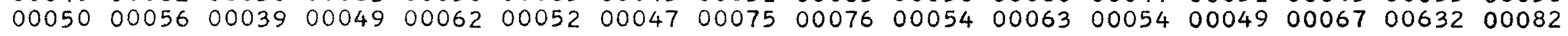
$000870008500080 \quad 000840008000091000860008100069000690008000100 \quad 00073 \quad 0009300098 \quad 00060$ $00091000630007900064000730008100090 \quad 003370008800084001020009600111001330013600125$ $0014200140 \quad 0015700181001430004100031000190000900004000070001600010000090002400777$

CRYSTAL HEIGHT $=01.000000$ IN. CRYSTAL RADIUS $=00.500000$ IN. $I=10000$ $I=10000 \quad A=00282$

$\begin{array}{lllllllllllllll}0 & 00042 & 00045 & 00043 & 00042 & 00048 & 00046 & 00041 & 00053 & 00066 & 00052 & 00040 & 00048 & 00045 & 00049\end{array}$ 00039000410003900049000420005100047000440005100060000490004900054000590005300056 $000410005400043 \quad 000550004200048 \quad 00066 \quad 00048 \quad 000490004200056 \quad 00052 \quad 00053 \quad 00046 \quad 00050 \quad 00046$ $0004700040 \quad 00056 \quad 00039000570003900058 \quad 00046 \quad 000440004800058 \quad 0004400048 \quad 000440004900047$ 00044000620004900045000430003600053000530005700057000420005700050000420005500053 $00040 \quad 00069000540005700048 \quad 00055 \quad 0003900053 \quad 0006200067000490005100070000650004100056$ $000530009501446 \quad 00148 \quad 0012900148 \quad 00103 \quad 001200011800141001280013800116001020011100074$ 00097006360013800106001080010400115001240013900052000980004600005000030000400289 $\begin{array}{ll}\text { INCIDENT ENERGY } & =04.450000 \text { MEV. } \\ \text { SOURCE HEIGHT } & =00.000000 \text { IN. }\end{array}$ $P=42199$

INCIDENT ENERGY $=01.330000$ MEV. SOURCE HEIGHT $=00.000000$ IN.
\[ =28101 \]

INCIDENT ENERGY $=02.620000 \mathrm{MEV}$. SOURCE HEIGHT $=00.000000$ IN.

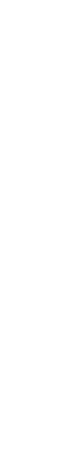


CRYSTAL HEIGHT $=01.000000$ IN. CRYSTAL RADIUS $=00.500000 \mathrm{IN}$. $I=10000$ $A=00127$

0003700031000320005500045 $00056 \quad 0003900038 \quad 000440003900054$ 000390004600051000530005900058 $0005500046 \quad 00046 \quad 00044000490004500064$ 000360004800041000470003800059 $\begin{array}{llllllllll}00075 & 00048 & 00052 & 00062 & 00071 & 00076 & 00058\end{array}$ 0019500173000970013600088
INCIDENT ENERGY $=06.130000 \mathrm{MEV}$. SOURCE HEIGHT $=00.000000$ IN. $P=41701$ $0003900038 \quad 00055000370005200053 \quad 000650004500043$ $00050 \quad 0003400040 \quad 00053 \quad 000410004900030 \quad 0004400041$ $\begin{array}{lllllllllll}0 & 0037 & 00061 & 00035 & 00044 & 00043 & 00048 & 00043 & 00048 & 00051\end{array}$ $0004900057000550004800046 \quad 0004300050 \quad 0004900041$ $\begin{array}{lllllllllll}0 & 0054 & 00052 & 00047 & 00045 & 00041 & 00046 & 00040 & 00047 & 00048\end{array}$ $\begin{array}{lllllllllll}00046 & 00051 & 00050 & 00060 & 00056 & 00052 & 00047 & 00062 & 00070\end{array}$ $\begin{array}{llllllllll}00132 & 00215 & 00086 & 01642 & 00212 & 00182 & 00184 & 00200 & 00222\end{array}$ 000690007500099000770003000029000040000200132
CRYSTAL HEIGHT $=01.000000 \mathrm{IN}$. CRYSTAL RADIUS $=00.500000$ IN.

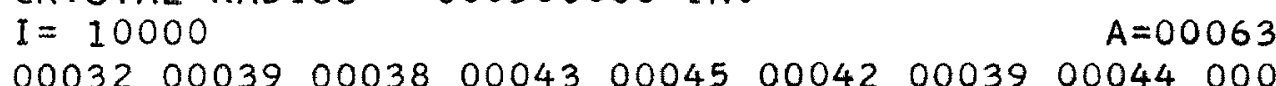

$\begin{array}{lllllll}00032 & 00039 & 00038 & 00043 & 00045 & 00042 & 00039 \\ 00053 & 00051 & 00047 & 00047 & 00044 & 00045 & 00\end{array}$

$000460003700039000390004500040 \quad 00048$

$00040 \quad 00048 \quad 00040 \quad 00042000530005400085$

$0003700053 \quad 0004700046 \quad 00052 \quad 00048 \quad 00052$

$0005700040 \quad 0006200051000450004400052$

00055000600006500078000450006600067

$00195002220017400166 \quad 0026100056 \quad 00538$
INCIDENT ENERGY $=07.100000 \mathrm{MEV}$ SOURCE HEIGHT $=00.000000$ IN.

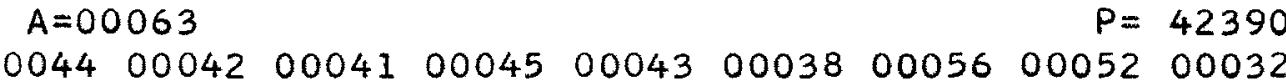
$\begin{array}{llllllllll}0 & 0042 & 00043 & 00048 & 00043 & 00043 & 00055 & 00039 & 00046 & 00051\end{array}$ $00048 \quad 00050 \quad 0004100041000470005500046 \quad 0004400044$ $\begin{array}{llllllllll}00057 & 00056 & 00038 & 00035 & 00053 & 00050 & 00045 & 00040 & 00044\end{array}$ $000430004100048 \quad 00048 \quad 00058 \quad 00059000450005200040$ $\begin{array}{llllllllll}00058 & 00044 & 00062 & 00040 & 00057 & 00052 & 00048 & 00054 & 00056\end{array}$ $\begin{array}{llllllllll}00056 & 00195 & 00066 & 00088 & 00591 & 00142 & 01417 & 00286 & 00220\end{array}$ $000710008300050 \quad 0004700056 \quad 0002100012 \quad 00000 \quad 00063$
CRYSTAL HEIGHT $=01.000000$ IN. CRYSTAL RADIUS $=00.500000$ IN. $I=10000$

$00048 \quad 0004200042 \quad 00048 \quad 000540005500042$ 00048000460006300060000520004200053 00051000490005600046000520005100056

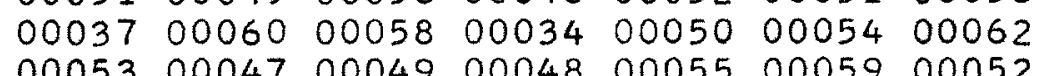
$\begin{array}{lllllll}00053 & 00047 & 00049 & 00048 & 00055 & 00059 & 00052 \\ 00053 & 00056 & 00058 & 00049 & 00058 & 00060 & 00054\end{array}$ 00058000600007300073000600006900091 00270002660026200180002670016400048
INCIDENT ENERGY $=08.000000 \mathrm{MEV}$. SOURCE HEIGHT $=00.000000$ IN. $A=00051$ $P=46720$ $0004200068 \quad 00040 \quad 0004900048 \quad 00062000620005500055$ $\begin{array}{lllllllllll}00062 & 00044 & 00067 & 00056 & 00045 & 00053 & 00045 & 00046 & 00068\end{array}$ $00057000490006400051 \quad 000470005200052 \quad 00056 \quad 00056$ $\begin{array}{lllllllllll}00061 & 00068 & 00057 & 00053 & 00055 & 00061 & 00063 & 00048 & 00041\end{array}$ $00058000450007100053 \quad 00052 \quad 00051 \quad 00073 \quad 0005400056$ $\begin{array}{lllllllllll}0 & 0053 & 00050 & 00059 & 00061 & 00051 & 00056 & 00057 & 00059 & 00072\end{array}$ $00078 \quad 00083 \quad 00126 \quad 00180 \quad 00139005090040100158 \quad 01438$ 005450006300042000420003400018000230000000053 
TABLE I (CONTINUED)

CRYSTAL HEIGHT $=01.000000$ IN. CRYSTAL RACIUS $=00.500000$ IN. $I=10000$ $0004200042000490004500041 \quad 0004400048000420004600056000400004800054000380003500057$ $00050 \quad 00052 \quad 00043 \quad 00050 \quad 000420004500042 \quad 00046 \quad 00050 \quad 00050 \quad 000390004500040 \quad 0003700060 \quad 00048$ $0004400041000490005200060 \quad 0005800051000540005200077000650006200058000580005200070$ $00056 \quad 00057 \quad 00052 \quad 00048 \quad 00048 \quad 0005 C \quad 00051 \quad 0004900063 \quad 00055000590006200050 \quad 0006900054 \quad 00039$ 00049000560005200044000540006500051000460005900059000450006100057000560005500051

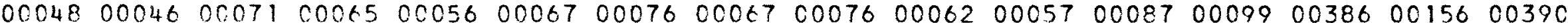
$00730 \quad 0022100812002530023700106 \quad 003790008500044002970004300021000210003100003 \quad 00025$

CRYSTAL HEICHT $=$ CI.OCOOOR IN. CRYSTAL RACIUS $=0.5000 C C$ IN. $I=10000$

00031000440003700049000530004500052000500004600055000420005100064000540005600053 $00050 \quad 0004100048 \quad 0004700060 \quad 00038 \quad 00048 \quad 0004400040 \quad 0005100052 \quad 00056 \quad 00046 \quad 000550006400062$ $00042000540004300064000610006500055 \quad 06 C 460004900044000560005300049000500004700055$ $0005200056 \quad 00047 \quad 00055 \quad 00055 \quad 00054 \quad 00071 \quad 00076 \quad 00066 \quad 000620006900057 \quad 00076 \quad 0005900056 \quad 00065$ $0005900037 \quad 0006900058 \quad 0005500050 \quad 00046000580006100055000600006000048000540006400054$ $00046 \quad 00056 \quad 00060 \quad 000640006500058 \quad 000630004900072 \quad 00070 \quad 0006000050000730066100056 \quad 00063$ $00046 \quad 000650006200061 \quad 00070 \quad 00060 \quad 00062 \quad 0006400048 \quad 00030000310008200068 \quad 0031400402 \quad 00346$ $003690055900411 \quad 002610030200430 \quad 00088082550008100032000920001500024000090000500009$

CRYSTAL HEIGHT $=01.000000$ IN. CRYSTAL RADIUS $=0.0 .500000$ IN. $I=10000$ $A=00003$

INCIDENT ENERGY $=14.000000 \mathrm{MEV}$. SOURCE HEIGHT $=00.000000$ IN.

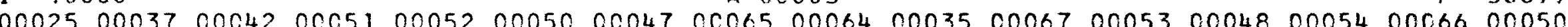
$00059000420006400045 \quad 00062 \quad 00051 \quad 00042 \quad 00646 \quad 00060 \quad 0005900056 \quad 00050 \quad 00045000340006400053$ $00051 \quad 00056 \quad 00 C 42 \quad 00050 \quad 0005900056 \quad c 0040 \quad c 0054 \quad 00064 \quad 0004900054 \quad 00056 \quad 000490004700052 \quad 00054$ $00053000630006900057 \quad C C C 7400078 \quad 0 C C 75 \quad 0 C 08500078 \quad 0007900076 \quad 000590008100076 \quad 0005100070$ $000580006100070 \quad 00046 \quad 00068 \quad 0006900062 \quad 000670008100065000750006900071000850006900073$ $000790005900076 \quad 0006100058 \quad 0006100060 \quad 000650006100063000550004200055000650005500047$ $0005400060 \quad 00071 \quad 00055 \quad 00078 \quad 0006500057 \quad 00035000160003200050 \quad 00169001740040500276 \quad 00461$ $0033100390 \quad 00307003220028100210 \quad 00152001220007100050 \quad 000240002000010000020000100003$ 
TABLE I (CONTINUED)

CRYSTAL HEIGHT $=02.000000$ IN. CRYSTAL RADIUS $=01.000000$ IN. $I=10000$

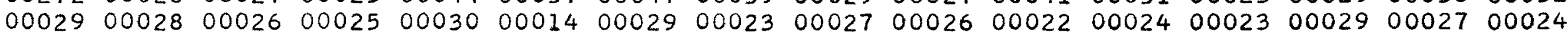
00018000200001300019000190001400018000200002000019000150001400011000160001900017 $00013000210002000010 \quad 00018 \quad 00020 \quad 000290001900020000290002300026 \quad 000180002400030 \quad 00027$ $0002100034000220000400006 \quad 0000500005000050000300001000020000200000000050000200001$ $000010000100000000000000100000000000000000000000000000000000000000000000000 \quad 00000$ $000000000000000000000000000000 \quad 00000 \quad 0000000000000000000000000000000000000000 \quad 00000$ 00000000000000000000001160000600000000000000000000000000000000000000000000007963

CRYSTAL HEIGHT $=02.000000$ IN. CRYSTAL RADIUS $=01.000000$ IN. $I=10000$

$00197000650006200062000640007300056 \quad A=04570$ $00048 \quad 00058 \quad 000520006200061000620006400053 \quad 00057$ $00055 \quad 00056 \quad 00052 \quad 00053 \quad 0004900056 \quad 00058 \quad 0004500051$ $0005500050 \quad 000470006100037000480004800047000$ $\begin{array}{lllllllllll}00039 & 00054 & 00042 & 00048 & 00058 & 00054 & 00059 & 00043 & 00079 & 00 \\ 00047 & 00060 & 00044 & 00063 & 00064 & 00050 & 00069 & 00071 & 00074 & 00\end{array}$

$00010 \quad 00010 \quad 0000700005000060000300005000070000100002000010000100002000000000000000$ $0000000000 \quad 0000000000 \quad 0000000000 \quad 00000 \quad 000000000000000000000003600000000000000004570$

CRYSTAL HEIGHT $=02.000000$ IN. CRYSTAL RADIUS $=01.000000$ IN. $I=10000$ $00069000620006300057000630006500059000740006400068000440004900058 \quad 000550005900050$ $00050 \quad 00062 \quad 00046 \quad 00048 \quad 00048 \quad 00042 \quad 00059000470006300056 \quad 00056 \quad 0006500057 \quad 0005400050 \quad 00060$ $00053000650005400060 \quad 00054 \quad 0004900067000540004600056 \quad 0005200058 \quad 00059000750005100064$ $\begin{array}{lllllllllllllllll}00059 & 00047 & 00069 & 00057 & 00060 & 00074 & 00046 & 00060 & 00065 & 00059 & 00066 & 00070 & 00061 & 00063 & 00071 & 00066\end{array}$ $00061000650007700073 \quad 0007200068 \quad 00072000670006900059000610008000081000650009000090$

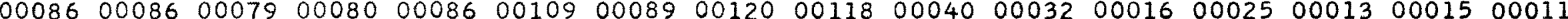
00007000050000500000000010000100000000000000000000000010000000001000190000103136

INCIDENT ENERGY $=01.170000 \mathrm{MEV}$. SOURCE HEIGHT $=00.000000$ IN. $P=10881$

INCIDENT ENERGY $=00.661000 \mathrm{MEV}$ SOURCE HEIGHT $=00.000000$ IN. $P=06719$ 000510006300064000600005900057 000540005600052000470004300065 000710004800050000420004900049 000530005400057000570004300051 00007 00005 0000500000 00001 0000100000 00000 00000 00000 00001000000001000190000103136 
TABLE I (CONTINUED)

CRYSTAL HEIGHT $=02.000000$ IN CRYSTAL RADIUS $=01.000000$ IN. $I=10000$ 00000340003600037000360004200034000390003800033000290003300041000380004000033 (1) $0003300039000310004600033000410004400040 \quad 0004900042000310005100030000270003500041$ $0004300045000450001700053 \quad 00038000370003000035000460004300042000410004200050 \quad 00043$ $000410003700040 \quad 0003900047000330003800050 \quad 0004000050 \quad 000420003700046000460003200046$ $000350004400043 \quad 00060 \quad 000470004900051 \quad 0004300050 \quad 000410004000047000380005400053 \quad 00046$ $\begin{array}{lllllllllllllllll}00063 & 00073 & 00847 & 00146 & 00129 & 00167 & 00142 & 00138 & 00144 & 00159 & 00109 & 00124 & 00088 & 00093 & 00090 & 00091\end{array}$ $00102010570019200135001490015600140 \quad 001550015700079001150006800017000050000501022$

INCIDENT ENERGY $=04.450000 \mathrm{MEV}$ SOURCE HEIGHT $=00.000000$ IN. $P=18993$
CRYSTAL HEIGHT $=02.0000001 \mathrm{~N}$. CRYSTAL RADIUS $=01.000000$ IN. $I=10000$ 00129000520004900054000510004400054 $0006300040 \quad 00066 \quad 00057000520005900055$ 00055000560005100046000500004700061 $000390006400047000590005900056 \quad 00054$ $00068 \quad 00060 \quad 0005100064000650004700063$ $0006200069000670006200076 \quad 00067 \quad 00075$ $\begin{array}{llllllll}00093 & 00092 & 00079 & 00090 & 00083 & 00077 & 00126 \\ 00013 & 00007 & 00005 & 00005 & 00006 & 00003 & 00000\end{array}$ 000130000700005

CRYSTAL HEIGHT $=02.000000$ IN. CRYSTAL RADIUS $=01.000000 \mathrm{IN}$. $I=10000$

$\begin{array}{llllllllllllll}0 & 0 & \end{array}$ $000460003900050 \quad 0005300038 \quad 000370005200059000410005900050 \quad 0003500055000590006100056$ $000440006000050 \quad 00040 \quad 0004400057000540003700059000540004900049000470004500063 \quad 00049$ $\begin{array}{llllllllllllllll}00060 & 00058 & 00051 & 00043 & 00053 & 00055 & 00055 & 00045 & 00065 & 00044 & 00059 & 00053 & 00062 & 00058 & 00418 & 00071\end{array}$ $00075 \quad 000720008500095 \quad 000670005200088000790007100070 \quad 00076 \quad 0006400075000740008200074$ $000580006900091000770009100073 \quad 00078 \quad 004970009600096001000010000116001130013100120$ $001490011000150001510012200040 \quad 00032000240002700006000030000800007000060001001722$ INCIDENT ENERGY $=01.330000$ MEV. SOURCE HEIGHT $=00.000000$ IN.
$\mathrm{P}=11941$ 000600006900045000580005600056000520006300055 $\begin{array}{llllllllll}00053 & 00049 & 00051 & 00054 & 00044 & 00043 & 00078 & 00055 & 00051\end{array}$ $\begin{array}{lllllllll}00053 & 00055 & 00046 & 00063 & 00051 & 00051 & 00056 & 00046 & 00048\end{array}$ 000640007100058000670006900064000720008200066 $\begin{array}{llllllllll}00103 & 00110 & 00129 & 00119 & 00052 & 00040 & 00040 & 00028 & 00011\end{array}$ INCIDENT ENERGY $=02.620000 \mathrm{MEV}$. SOURCE HEIGHT $=00.000000$ IN. $P=16452$ 
CRYSTAL HEIGHT $=02.000000$ IN. CRYSTAL RADIUS $=01.000000$ IN. $I=10000$ $000320003900028 \quad 0003200030 \quad 00036 \quad 000280002700035$ $0004100036 \quad 00030 \quad 000250003200020 \quad 000380002500020$ 000370003500034000310003600051000370004100038 $\begin{array}{llllllllll}00033 & 00037 & 00036 & 00028 & 00033 & 00033 & 00039 & 00029 & 00027\end{array}$ $000320003800035000470005200046 \quad 000340004100030$ 000390005000049000520005600059 002050017900125001310010501372
INCIDENT ENERGY $=06.130000 \mathrm{MEV}$ SOURCE HEIGHT $=00.000000$ IN. $P=18885$
002900032 00042000400002200020000370002900032 $0003900028 \quad 0003900028 \quad 00040 \quad 0003100029$ $\begin{array}{lllllll}00024 & 00030 & 00025 & 00039 & 00034 & 00027 & 00032\end{array}$ $\begin{array}{llllllll}00035 & 00035 & 00033 & 00036 & 00042 & 00046 & 00048\end{array}$ $\begin{array}{llllllll}0 & 0041 & 00035 & 00033 & 00035 & 00033 & 00023 & 00042\end{array}$ 00039000560004600038000660004300051 $\begin{array}{llllllllll}0062 & 00073 & 00105 & 00080 & 01111 & 00262 & 00221 & 00239 & 00203 & 00215\end{array}$ $\begin{array}{llllllllll}0163 & 00142 & 00178 & 00239 & 00166 & 00078 & 00070 & 00008 & 00005 & 00621\end{array}$
CRYSTAL HEIGHT $=02.000000$ IN. CRYSTAL RADIUS $=01.000000 \mathrm{IN}$. $I=10000$ $00020 \quad 00029000290002400038 \quad 000340003200023000310003100040 \quad 0003400034000320002600029$ $\begin{array}{lllllllllllllllll}00028 & 00034 & 00020 & 00023 & 00037 & 00038 & 00031 & 00028 & 00035 & 00023 & 00025 & 00024 & 00025 & 00018 & 00038 & 00026\end{array}$

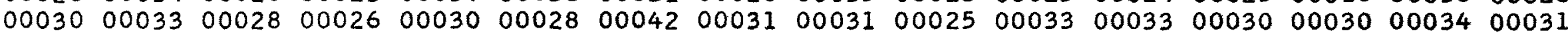
$\begin{array}{lllllllllllllllll}0 & 0028 & 00040 & 00028 & 00036 & 00034 & 00037 & 00036 & 00027 & 00045 & 00031 & 00034 & 00027 & 00030 & 00049 & 00039 & 00044\end{array}$

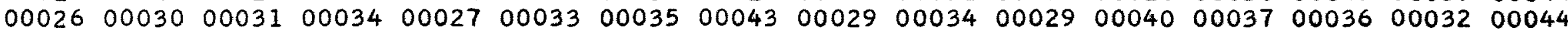

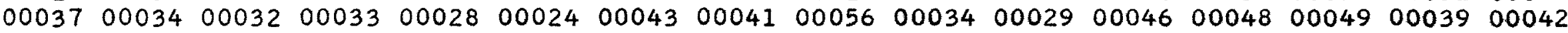

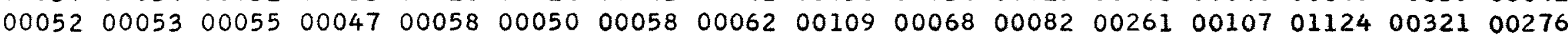
$00268002300023300170 \quad 0026500108 \quad 01370 \quad 001570020600168001420013300084000330000100495$

CRYSTAL HEIGHT $=02.000000$ IN. CRYSTAL RADIUS $=01.000000$ IN. $I=10000$ $\begin{array}{llllllllllllllllll}I=1 & 10000 \\ 00023 & 00020 & 00024 & 00026 & 00035 & 00028 & 00019 & 00027 & 00024 & 00030 & 00024 & 00025 & 00040 & 00023 & 00026 & 00031 \\ 00032 & 00019 & 00027 & 00029 & 00024 & 00015 & 00027 & 00031 & 00021 & 00030 & 00044 & 00025 & 00024 & 00030 & 00040 & 00022 \\ 00020 & 00031 & 00025 & 00029 & 00032 & 00025 & 00027 & 00032 & 00038 & 00026 & 00033 & 00022 & 00030 & 00024 & 00035 & 00033 \\ 00028 & 00025 & 00036 & 00023 & 00043 & 00025 & 00032 & 00032 & 00043 & 00035 & 00035 & 0.0035 & 00029 & 00038 & 00027 & 00026 \\ 00036 & 00031 & 00027 & 00041 & 00026 & 00032 & 00031 & 00030 & 00025 & 00049 & 00035 & 00030 & 00034 & 00035 & 00040 & 00033 \\ 00032 & 00027 & 00039 & 00032 & 00040 & 00035 & 00052 & 00041 & 00033 & 00035 & 00039 & 00038 & 00040 & 00045 & 00045 & 00037 \\ 00050 & 00041 & 00057 & 00050 & 00058 & 00076 & 00063 & 00059 & 00073 & 00084 & 00089 & 00097 & 00279 & 00242 & 00115 & 01089 \\ 00331 & 00359 & 00332 & 00268 & 00332 & 00192 & 00096 & 01416 & 00193 & 00143 & 00136 & 00122 & 00062 & 00073 & 00004 & 00435\end{array}$

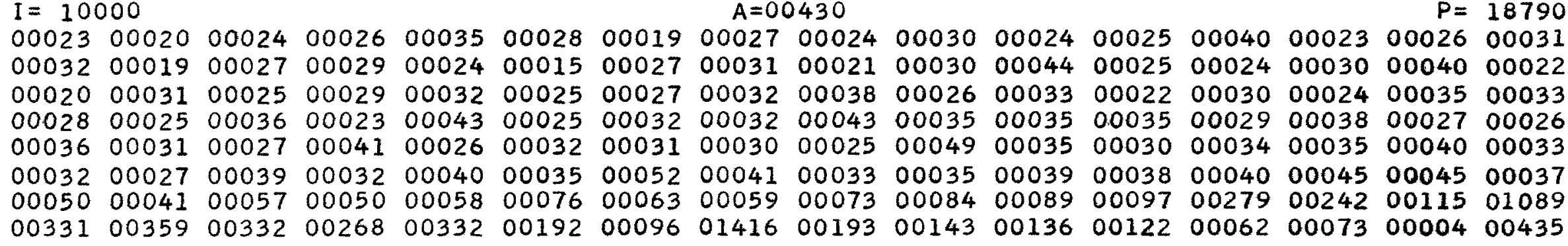

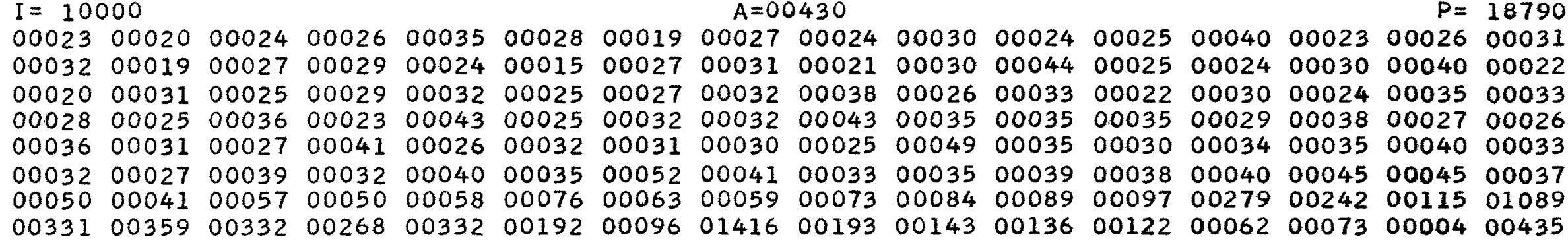

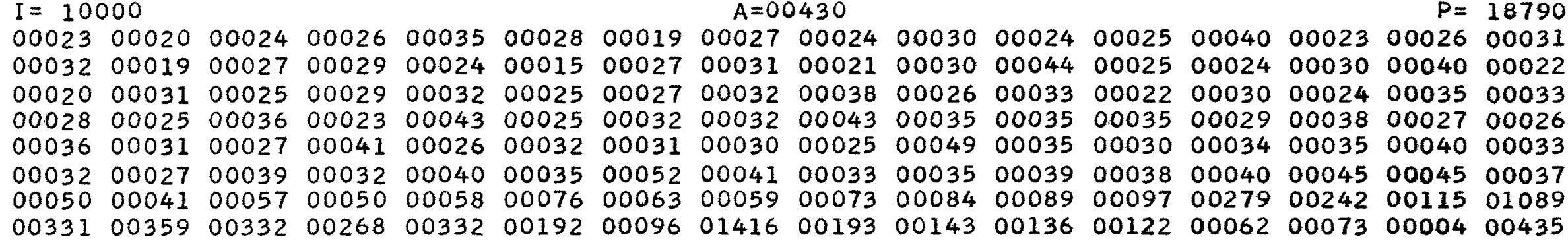

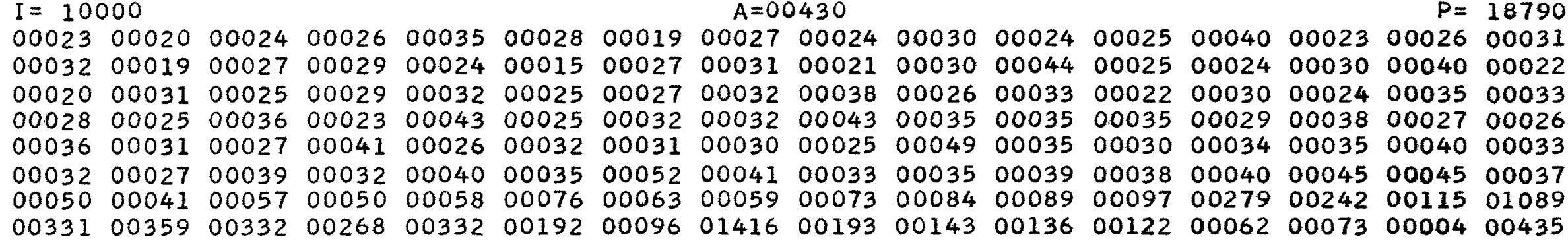
$\begin{array}{llllllllllllllllll}I=1 & 10000 \\ 00023 & 00020 & 00024 & 00026 & 00035 & 00028 & 00019 & 00027 & 00024 & 00030 & 00024 & 00025 & 00040 & 00023 & 00026 & 00031 \\ 00032 & 00019 & 00027 & 00029 & 00024 & 00015 & 00027 & 00031 & 00021 & 00030 & 00044 & 00025 & 00024 & 00030 & 00040 & 00022 \\ 00020 & 00031 & 00025 & 00029 & 00032 & 00025 & 00027 & 00032 & 00038 & 00026 & 00033 & 00022 & 00030 & 00024 & 00035 & 00033 \\ 00028 & 00025 & 00036 & 00023 & 00043 & 00025 & 00032 & 00032 & 00043 & 00035 & 00035 & 0.0035 & 00029 & 00038 & 00027 & 00026 \\ 00036 & 00031 & 00027 & 00041 & 00026 & 00032 & 00031 & 00030 & 00025 & 00049 & 00035 & 00030 & 00034 & 00035 & 00040 & 00033 \\ 00032 & 00027 & 00039 & 00032 & 00040 & 00035 & 00052 & 00041 & 00033 & 00035 & 00039 & 00038 & 00040 & 00045 & 00045 & 00037 \\ 00050 & 00041 & 00057 & 00050 & 00058 & 00076 & 00063 & 00059 & 00073 & 00084 & 00089 & 00097 & 00279 & 00242 & 00115 & 01089 \\ 00331 & 00359 & 00332 & 00268 & 00332 & 00192 & 00096 & 01416 & 00193 & 00143 & 00136 & 00122 & 00062 & 00073 & 00004 & 00435\end{array}$

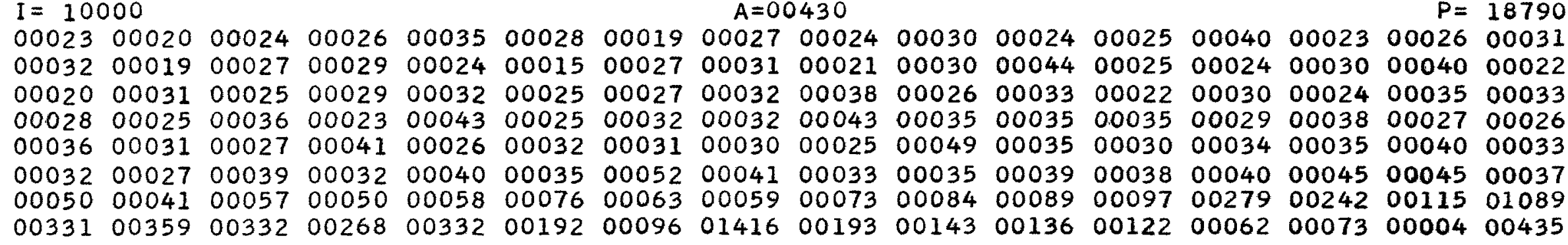

INCIDENT ENERGY $=08.000000 \mathrm{MEV}$. SOURCE HEIGHT $=00.000000$ IN. $P=18790$ 
TABLE I (CONTINUED)

CRYSTAL HEIGHT $=02.000000$ IN. CRYSTAL RADIUS $=01.000000$ IN. $I=10000$

000210002200015000200001700020000300003400034

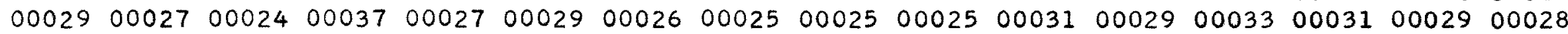
$\begin{array}{lllllllllllllllll}00026 & 00025 & 00032 & 00024 & 00033 & 00021 & 00030 & 00025 & 00025 & 00027 & 00028 & 00029 & 00019 & 00022 & 00030 & 00027\end{array}$ $00031000380004400025000270001900030 \quad 000350004600041000350003300043000450003100036$ $\begin{array}{lllllllllllllllll}0 & 0024 & 00034 & 00031 & 00029 & 00029 & 00055 & 00034 & 00036 & 00033 & 00043 & 00032 & 00028 & 00033 & 00031 & 00028 & 00045\end{array}$

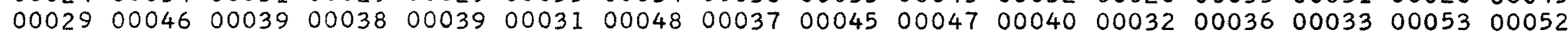
$\begin{array}{llllllllllllllllll}0 & 0 & 0 & 0051 & 00047 & 00038 & 00054 & 00059 & 00067 & 00070 & 00071 & 00077 & 00061 & 00087 & 00104 & 00205 & 00117 & 00221\end{array}$ $0049200227009190039700390 \quad 0026300644002160010201090001730010400113001250000900324$

CRYSTAL HEIGHT $=02.000000 \mathrm{IN}$. CRYSTAL RADIUS $=01.000000$ IN. $I=10000$

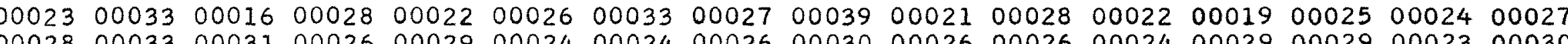

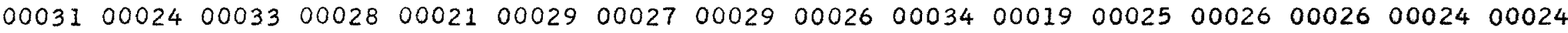

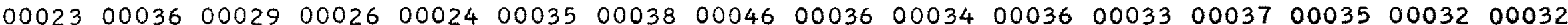

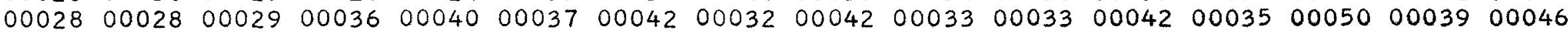

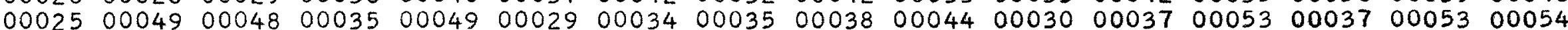

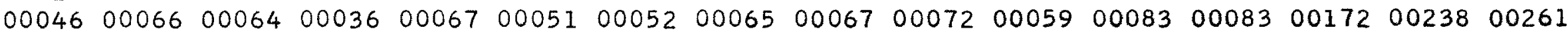
$0028600495 \quad 004550030900449007590026800806 \quad 0028100129006840010800163000840001900172$

CRYSTAL HEIGHT $=02.000000 \mathrm{IN}$ CRYSTAL RADIUS $=01.000000$ IN. $I=10000$

10017000170002500022000300002200032004 $0002400022 \quad 00024 \quad 0002900034 \quad 000290002900027 \quad 0002800023000310002500027 \quad 0002500025 \quad 00026$ $\begin{array}{llllllllllllllll}00026 & 00027 & 00025 & 00027 & 00018 & 00022 & 00026 & 00028 & 00021 & 00030 & 00018 & 00034 & 00028 & 00027 & 00030 & 00024\end{array}$

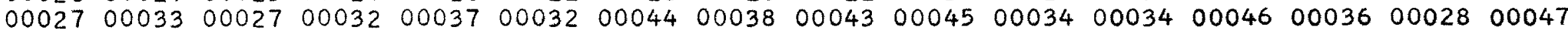

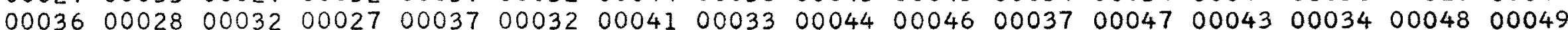
$00050 \quad 00048 \quad 000290004200053 \quad 00040 \quad 00045000450003600040 \quad 0005900046 \quad 00043000520003700032$ $\begin{array}{llllllllllllllllll}00050 & 00053 & 00059 & 00063 & 00051 & 00063 & 00061 & 00050 & 00052 & 00055 & 00049 & 00111 & 00137 & 00223 & 00239 & 00364\end{array}$

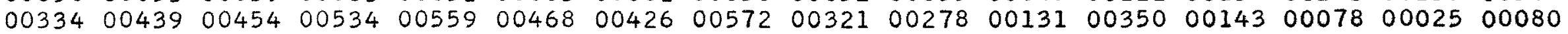

INCIDENT ENERGY $=14.000000$ MEV. SOURCE HEIGHT = $00.000000 \mathrm{IN}$. $P=15904$

INCIDENT ENERGY $=12.000000$ MEV. SOURCE HEIGHT $=00.000000$ IN. $P=16937$

INCIDENT ENERGY $=10.000000 \mathrm{MEV}$ SOURCE HEIGHT $=00.000000$ IN. $P=17697$

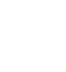

(n)


TABLE I (CONTINUED)

CRYSTAL HEIGHT $=04.000000$ IN. CRYSTAL RADIUS $=02.000000$ IN. $I=10000$ $\begin{array}{lllllllllllllllll} & 0 & 0 & 0\end{array}$ 00014000090001400009000120001300015000120000500014000110001200017000150002800021

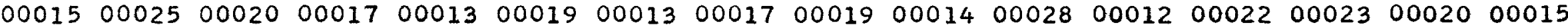
00025000380002900004000020000100001000010000000002000000000200000000020000300000 $0000100000 \quad 0000100000 \quad 0000100000 \quad 00000 \quad 00000 \quad 0000100000000000000000000 \quad 0000000000 \quad 00000$ $000000000000000000000000000000 \quad 00000 \quad 0000000000000000000000000000000000000000 \quad 00000$ 00000000000000000000000800000400000000000000000000000000000000000000000000008700

CRYSTAL HEIGHT $=04.000000$ IN. CRYSTAL RADIUS $=02.000000 \mathrm{IN}$ $I=10000$ $A=06268$ $00034000410003600036 \quad 00046 \quad 0003700030 \quad 0003300040 \quad 0003800036 \quad 0004200038 \quad 000290002900032$ $\begin{array}{llllllllllllllllll}00037 & 00042 & 00031 & 00037 & 00035 & 00041 & 00031 & 00037 & 00038 & 00033 & 00039 & 00045 & 00031 & 00033 & 00040 & 00037\end{array}$ $000370003100040 \quad 0003400047000450003500035000240002100036 \quad 0004300029000330003400043$ $0003400036 \quad 00028 \quad 0003900042 \quad 00024 \quad 00040 \quad 00037 \quad 00045000420003900048 \quad 0003400046 \quad 00040 \quad 00040$ $000410005300039000380004200039000360006100052000550005200050000290001500016 \quad 00014$ $0001000006 \quad 0000600007000020000200003000030000200000000010000000000 \quad 000000000000000$ $00000000000000000000000000000000000 \quad 000000000000000000000001600000000000000006268$

CRYSTAL HEIGHT $=04.000000 \mathrm{IN}$ CRYSTAL RADIUS $=02.000000 \mathrm{IN}$. $I=10000$

$001160003800049000350004600043 \quad 00046 \quad 000350003800036 \quad 00042000430004500050 \quad 00043 \quad 00033$ $\begin{array}{llllllllllllllllll}00036 & 00039 & 00038 & 00048 & 00036 & 00032 & 00044 & 00036 & 00032 & 00039 & 00055 & 00037 & 00042 & 00041 & 00032 & 00052\end{array}$ $\begin{array}{llllllllllllllllll}00038 & 00033 & 00046 & 00043 & 00040 & 00045 & 00039 & 00053 & 00047 & 00051 & 00053 & 00035 & 00036 & 00031 & 00038 & 00036\end{array}$ $\begin{array}{llllllllllllllllll}00045 & 00043 & 00042 & 00034 & 00045 & 00045 & 00043 & 00030 & 00029 & 00048 & 00034 & 00044 & 00043 & 00049 & 00049 & 00045\end{array}$ $0002800041000510006200050 \quad 000540004100056 \quad 000710004300050 \quad 0004800055000430004900053$ $\begin{array}{llllllllllllllllllll}00050 & 00053 & 00051 & 00045 & 00055 & 00047 & 00060 & 00062 & 00051 & 00057 & 00074 & 00062 & 00061 & 00061 & 00054 & 00065\end{array}$

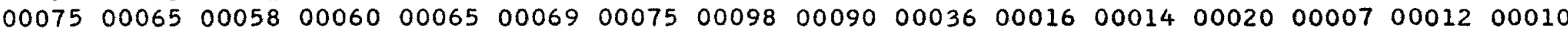
00002000030000400002000020000000000000000000100000000000000000001000130000004790

INCIDENT ENERGY $=01.170000$ MEV. SOURCE HEIGHT $=00.000000$ IN $P=03729$ INCIDENT ENERGY $=00.661000 \mathrm{MEV} \bullet$ SOURCE HEIGHT $=00.000000$ IN. $P=02012$ $\begin{array}{ll}\text { INCIDENT ENERGY } & =00.279000 \text { MEV. } \\ \text { SOURCE HEIGHT } & =00.000000 \text { IN. }\end{array}$ 0 
TABLE I (CONTINUED)

CRYSTAL HEIGHT $=04.000000 \mathrm{IN}$. CRYSTAL RADIUS $=02.000000 \mathrm{IN}$. $I=10000$ $0004200040 \quad 0004700034000440003700033$ $00038 \quad 0003500048 \quad 000420004900052 \quad 00043$ $00040 \quad 000430004100042000380003700044$ $0004300050 \quad 0002500048 \quad 000470005600039$ $\begin{array}{lllllllll}00047 & 00050 & 00054 & 00045 & 00054 & 00067 & 00069 & 004 \\ 00067 & 00057 & 00074 & 00076 & 00070 & 00075 & 00075 & 006\end{array}$

$A=04597$ $0034000560002900040 \quad 0004300036000410004300033$ $\begin{array}{llllllllll}00038 & 00039 & 00033 & 00034 & 00040 & 00043 & 00044 & 00033 & 00040\end{array}$ $\begin{array}{llllllllll}00036 & 00037 & 00050 & 00059 & 00046 & 00036 & 00048 & 00033 & 00044\end{array}$ $00050 \quad 00043 \quad 00046 \quad 00042 \quad 00042 \quad 0003400042 \quad 00038 \quad 00034$ $000380004900058 \quad 00053 \quad 00051 \quad 00053 \quad 00057 \quad 0008300044$ $000530005900053 \quad 00070 \quad 0005400060000650006300069$
CRYSTAL HEIGHT $=04.000000$ IN. CRYSTAL RADIUS $=02.000000$ IN. $I=10000$

$A=02515$ $\begin{array}{llllllllllllll} & 0 & 0 & 0 & 0\end{array}$ $\begin{array}{lllllllllllllllllll}0 & 0 & 0 & 0 & 0 & 00031 & 00017 & 00037 & 00022 & 00030 & 00027 & 00024 & 00028 & 00030 & 00022 & 00021 & 00035 & 00023 & 00031\end{array}$ $00028000190003200026 \quad 00031 \quad 00028 \quad 00024 \quad 0002500031000230001900024000300002500022 \quad 00027$

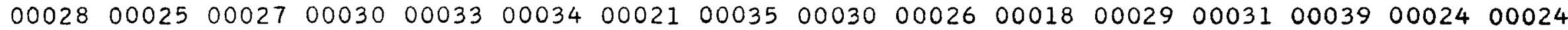

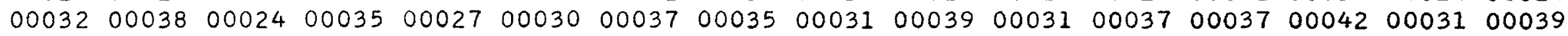
$00036 \quad 00040 \quad 00028 \quad 000250004400051 \quad 0004500040 \quad 00046 \quad 00036 \quad 0004500058 \quad 00056 \quad 000490004700052$ $00046000530034900103 \quad 001040013200103001000010200108001050009600097000800008400080$ 00102013360017300164001660016900160001300016800079001000005800011000020000402524

INCIDENT ENERGY $=04.450000$ MEV. SOURCE HEIGHT $=00.000000$ IN. $\begin{array}{lllllllllllllllll}00067 & 00057 & 00074 & 00076 & 00070 & 00075 & 00075 & 00072 & 00088 & 00085 & 00090 & 00054 & 00030 & 00029 & 00016 & 00013 \\ 00007 & 00009 & 00004 & 00007 & 00001 & 00002 & 00000 & 00001 & 00000 & 00001 & 00001 & 00001 & 00000 & 00011 & 00001 & 04597\end{array}$ $\begin{array}{lllllllllllllllll}00067 & 00057 & 00074 & 00076 & 00070 & 00075 & 00075 & 00072 & 00088 & 00085 & 00090 & 00054 & 00030 & 00029 & 00016 & 00013 \\ 00007 & 00009 & 00004 & 00007 & 00001 & 00002 & 00000 & 00001 & 00000 & 00001 & 00001 & 00001 & 00000 & 00011 & 00001 & 04597\end{array}$ $\begin{array}{llllllllllllllllll}00067 & 00057 & 00074 & 00076 & 00070 & 00075 & 00075 & 00072 & 00088 & 00085 & 00090 & 00054 & 00030 & 00029 & 00016 & 00013 \\ 00007 & 00009 & 00004 & 00007 & 00001 & 00002 & 00000 & 00001 & 00000 & 00001 & 00001 & 00001 & 00000 & 00011 & 00001 & 04597\end{array}$

CRYSTAL HEIGHT $=04.000000$ IN.

CRYSTAL RADIUS $=02.000000$ IN. $I=10000$

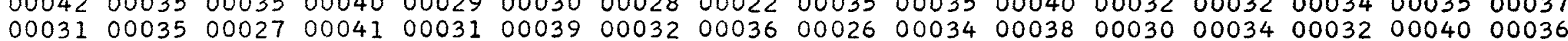
$000470003600041000310003500030 \quad 00050 \quad 00043 \quad 00040 \quad 000410003400032 \quad 00026000400004100032$ 00033000410003700042000450004400043000330003800033000380005300040000400005500042 $\begin{array}{lllllllllllllllllllllll}0 & 00036 & 00043 & 00041 & 00031 & 00044 & 00043 & 00048 & 00041 & 00030 & 00036 & 00032 & 00051 & 00047 & 00034 & 00167 & 00067\end{array}$ 00055000690006300063000650006200067000610006200060000530006800072000690008300063 $\begin{array}{llllllllllllllllll}0 & 00068 & 00084 & 00063 & 00077 & 00077 & 00085 & 00601 & 00091 & 00083 & 00107 & 00103 & 00103 & 00094 & 00122 & 00101\end{array}$ 00120001000010600118000940004900038000190001100007000000001000004000060001803204 $P=07494$
INCIDENT ENERGY $=02.620000$ MEV. SOURCE HEIGHT $=00.000000$ IN.

NCIDENT ENERGY $=01.330000 \mathrm{MEV}$ $\begin{array}{ll}\text { SOURCE HEIGHT } & =00.000000 \text { IN. }\end{array}$ 2 (n)

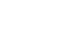

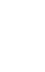


TABLE I (CONTINUED)

CRYSTAL HEIGHT $=04.000000$ IN. CRYSTAL RADIUS $=02.000000$ IN. $I=10000$

00020000150001300016000200001600021000120002400022000100002500026000150002200019 $\begin{array}{llllllllllllllll}00023 & 00020 & 00030 & 00024 & 00019 & 00024 & 00022 & 00025 & 00019 & 00033 & 00022 & 00017 & 00024 & 00024 & 00020 & 00022\end{array}$ $000170002500026 \quad 00013 \quad 000190002100032000270001900023000170002800025000190002100024$ $\begin{array}{lllllllllllllllll}00020 & 00026 & 00029 & 00023 & 00026 & 00032 & 00019 & 00029 & 00023 & 00020 & 00019 & 00021 & 00023 & 00022 & 00032 & 00026\end{array}$ $\begin{array}{lllllllllllllllll}00029 & 00026 & 00019 & 00030 & 00031 & 00022 & 00033 & 00027 & 00032 & 00033 & 00025 & 00024 & 00023 & 00041 & 00024 & 00025\end{array}$ $\begin{array}{lllllllllllllllll}00026 & 00025 & 00030 & 00023 & 00033 & 00040 & 00035 & 00022 & 00021 & 00032 & 00027 & 00031 & 00038 & 00041 & 00037 & 00043\end{array}$ $\begin{array}{lllllllllllllllll}0 & 00048 & 00027 & 00051 & 00043 & 00050 & 00035 & 00041 & 00064 & 00055 & 00042 & 00439 & 00161 & 00174 & 00155 & 00140 & 00168\end{array}$

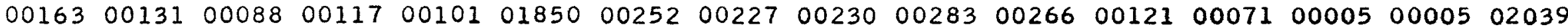

CRYSTAL HEIGHT $=04.000000$ IN. CRYSTAL RADIUS $=02.000000$ IN. $I=10000$ $00016 \quad 0002300015 \quad 00012000200002400019000220002200020000190001800019000140002100019$ 00018000170002200017000270002400014000150002200016000220001400019000250002700022 $00020 \quad 00022000240001500026 \quad 0002700023000230002200021000330002000019000210001500022$ $\begin{array}{llllllllllllllllll}00029 & 00029 & 00023 & 00026 & 00024 & 00026 & 00025 & 00025 & 00020 & 00023 & 00035 & 00028 & 00030 & 00025 & 00021 & 00027\end{array}$

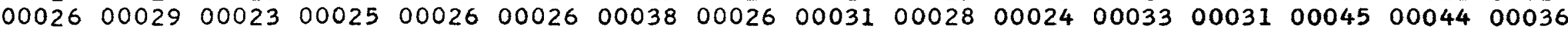
$\begin{array}{llllllllllllllllllll}00035 & 00041 & 00037 & 00045 & 00041 & 00033 & 00035 & 00060 & 00051 & 00052 & 00049 & 00107 & 00070 & 00501 & 00186 & 00193\end{array}$

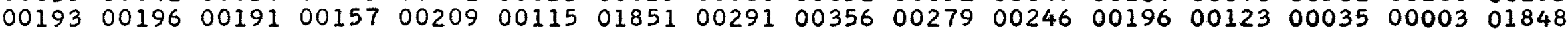

CRYSTAL HEIGHT $=04.000000$ IN. CRYSTAL RADIUS $=02.000000$ IN. $I=10000$

00012000100002000011000300002000017000090002300026000160002100022000110002100023

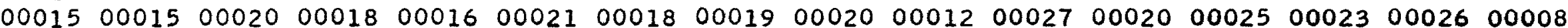

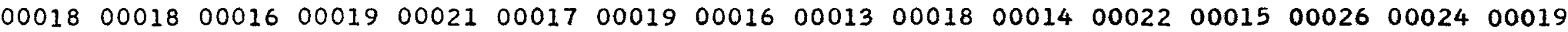
$\begin{array}{lllllllllllllllll}00021 & 00023 & 00018 & 00017 & 00025 & 00024 & 00010 & 00021 & 00019 & 00016 & 00018 & 00020 & 00026 & 00030 & 00018 & 00016\end{array}$

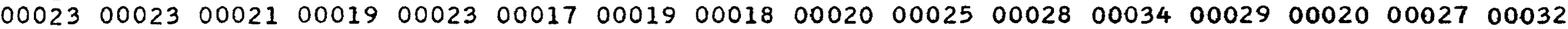

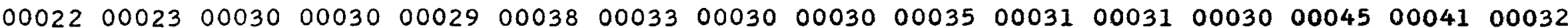
$\begin{array}{llllllllllllllll}00045 & 00030 & 00040 & 00040 & 00047 & 00042 & 00043 & 00040 & 00047 & 00060 & 00058 & 00057 & 00096 & 00106 & 00081 & 00544\end{array}$ $00213002290023400230 \quad 002680017000106 \quad 02040 \quad 0033800288002440024400148 \quad 0008300006 \quad 01683$
INCIDENT ENERGY $=08.000000$ MEV. SOURCE HEIGHT $=00.000000$ IN. P= 07536 INCIDENT ENERGY $=07.100000 \mathrm{MEV}$. SOURCE HEIGHT $=00.000000$ IN. $\begin{array}{ll}\text { INCIDENT ENERGY } & =06.130000 \mathrm{MEV} \bullet \\ \text { SOURCE HEIGHT } & =00.000000 \text { IN. }\end{array}$ 
TABLE I (CONTINUED)

CRYSTAL HEIGHT $=04.00000$ IN. CRYSTAL RADIUS $=02.000000$ IN. $I=10000$

00012000120001000014000190001500012

$000140001600016 \quad 0001200010 \quad 0001200019$

00013000110001700021000160001700020

000150000700021000210002300013000180001900021

$\begin{array}{lllllllllll}00025 & 00015 & 00030 & 00015 & 00020 & 00020 & 00020 & 00018 & 00017 & 006\end{array}$

$\begin{array}{llllllllll}00030 & 00037 & 00033 & 00029 & 00039 & 00044 & 00043 & 00054 & 00060 & 0\end{array}$ $A=01385$

INCIDENT ENERGY $=10.000000 \mathrm{MEV}$. SOURCE HEIGHT $=00.0 \cap C C O O \mathrm{IN}$. $P=07055$ 00012000200000900018000170002300013 $00012000120002000016 \quad 0001300026 \quad 00014$ $00027000170002000014 \quad 00020 \quad 00013 \quad 00019$ $0002700015000280001800014 \quad 0002300021$ $\begin{array}{lllllllll}00024 & 00036 & 00025 & 00027 & 00026 & 00031 & 00024\end{array}$ 00034000310002800035000320002300029 $000430005800054000690010000076 \quad 00131$

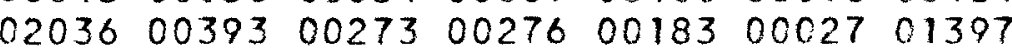

INCIDENT ENERGY $=12.000000 \mathrm{MEV}$.

CRYSTAL HEIGHT $=04.000000$ IN. CRYSTAL RADIUS $=02.000000$ IN. $I=10000$ SOURCE HEIGHT $=00.000000 \quad I N$.

$A=01180$ $P=06618$ $000140000900010 \quad 00010 \quad 000080001200018000160001800017000140001500020000140001200014$ $000150001400015 \quad 0001200013 \quad 00013000170000900018 \quad 00013000150002300016000130001200025$ $000200001400010 \quad 00010 \quad 000180000900019000120001200012000100001500014000150001200016$ $0001200016 \quad 0001500010 \quad 000190002200019000210001800022000180002100030000250002500020$ 00018000160002800027000190001800021000140002600020000220001400035000250001200022 $00027 \quad 000290001900027 \quad 0003100026 \quad 00024 \quad 000330002700026000170002800031000390003100034$ $000370003400030 \quad 000340003500038 \quad 000440003800055000520004700065000610008500125 \quad 00112$ $001380021300242001950029500669003000080200379002200158900336004430026600056 \quad 01184$

CRYSTAL HEIGHT $=04.000000$ IN. CRYSTAL RADIUS $=02.000000$ IN. $I=10000$

000060000900015000120001400011000090001800025 00016000150001700014000150001500012000150001800015000090001900011000180000800012 00011000120001100016000120001300016000210001600015000140001300014000160001200017 $0002300 C 220001000018000200002100014000190001300025000230001400020000210002300016$ $0001700023000130002100016 \quad 0001900021000210002200028000250002200036 \quad 0002500030 \quad 00026$ $0003700031 \quad 00027 \quad 00020 \quad 00022 \quad 00038 \quad 00028 \quad 00025000280002600026 \quad 00033000230003600027 \quad 00020$ $00025000340002700030 \quad 000430003200051 \quad 000440004200049000630006500077 \quad 001100009400153$ $001890019400275003540044500366004520073400530 \quad 0047900258 \quad 011610059100256 \quad 0 C 12100868$

INCICENT ENERGY $=14.000000 \mathrm{MEV}$. SOURCE HEIGHT $=00.000000$ IN. $P=06493$ 
CRYSTAL HEIGHT $=06.000000$ IN. CRYSTAL RADIUS $=03.000000$ IN. $I=10000$

$\begin{array}{llllllllllllllll}400005 & 00007 & 00004 & 00012 & 00006 & 00009 & 00008 & 00006 & 00010 & 00013 & 00016 & 00014 & 000130001300015\end{array}$ 00015000080001000014000090000900013000070001600006000140000900013000130000800013 $0000700011000110001300010 \quad 0001200017000120000900010000170001300016000180002000009$ 00015000150001200022000120001700020000180002100019000190002100019000280003200019 00025000190002300003000030000300003000030000000005000000000100002000000000100001 $0000400000 \quad 00000000000000000000 \quad 000000000100000000000000000000000000000000000 \quad 00000$ $00000 \quad 00000 \quad 00000 \quad 00000 \quad 00000 \quad 0000000000 \quad 000000000000000000000000000000000000000000000$ $00000 \quad 00000 \quad 0000000000 \quad 000870000500000 \quad 0000000000000000000000000000000000000000 \quad 08795$

CRYSTAL HEIGHT $=06.000000$ IN. CRYSTAL RADIUS $=03.000000 \mathrm{IN}$. $I=10000$ $A=07093$ $00120000270002200027000290002200027 \quad 000220002700015000250002800018000290002700032$ $\begin{array}{lllllllllllllllll}00028 & 00018 & 00028 & 00028 & 00030 & 00031 & 00030 & 00021 & 00026 & 00027 & 00026 & 00022 & 00020 & 00025 & 00023 & 00031\end{array}$ $\begin{array}{llllllllllllllllll}00028 & 00033 & 00017 & 00026 & 00024 & 00030 & 00030 & 00017 & 00034 & 00025 & 00033 & 00032 & 00028 & 00022 & 00031 & 00020\end{array}$

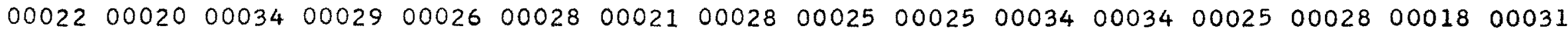
$00023000340002900037 \quad 00034 \quad 00030 \quad 00031 \quad 00030 \quad 00042 \quad 00036 \quad 00034 \quad 0003700032 \quad 000390002900034$ $0003400038 \quad 0003500050 \quad 00030 \quad 00043 \quad 000330004200050 \quad 00044000380005500023000120000900010$ $00001000080000800006 \quad 0000300005000020000100001000000000200001000000000100000 \quad 00000$ 00000000000000000000000000000000000000000000000000000000002200000000000000007093

CRYSTAL HEIGHT $=06.000000$ IN CRYSTAL RADIUS $=03.000000 \mathrm{IN}$. $I=10000$

00091000290003400028000320003100027000200002000036000290002200024000320003400020 $\begin{array}{lllllllllllllllll}00021 & 00028 & 00029 & 00034 & 00030 & 00033 & 00029 & 00031 & 00034 & 00037 & 00024 & 00025 & 00033 & 00037 & 00026 & 00027\end{array}$ $0003200036 \quad 00032000320002400036 \quad 00034000290002200033000240003600033000240003400036$ $\begin{array}{lllllllllllllllll}00034 & 00036 & 00028 & 00025 & 00041 & 00029 & 00040 & 00029 & 00030 & 00039 & 00035 & 00031 & 00046 & 00028 & 00036 & 00038\end{array}$ $0004300036 \quad 0003100035000340003700050 \quad 00045000590003100038 \quad 00040 \quad 000420004100045 \quad 00046$

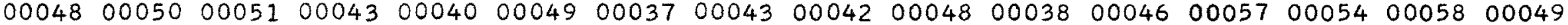
$0005600048 \quad 00061000530005200070 \quad 00072000710006200025000220001300019000110000900012$ 00005000010000100001000010000000000000010000000000000000000000000000050000105893
INCIDENT ENERGY $=00.661000 \mathrm{MEV}$ SOURCE HEIGHT $=00.000000$ IN. $\begin{array}{ll}\text { INCIDENT ENERGY } & =01.170000 \mathrm{MEV} \\ \text { SOURCE HEIGHT } & =00.000000 \mathrm{IN}\end{array}$ P 01777 $\begin{array}{ll}\text { INCIDENT ENERGY } & =00.279000 \mathrm{MEV} \\ \text { SOURCE HEIGHT } & =00.000000 \mathrm{IN}\end{array}$ 
CRYSTAL HEIGHT $=06.000000$ IN. CRYSTAL RADIUS $=03.000000 \mathrm{IN}$. $I=10000$

000720002300029000300002900028000230002300032

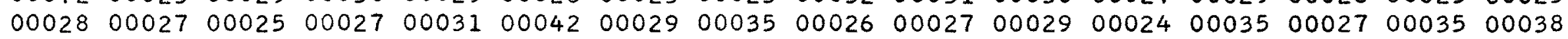
$\begin{array}{llllllllllllllllll}00033 & 00030 & 00020 & 00023 & 00034 & 00033 & 00027 & 00033 & 00026 & 00036 & 00029 & 00038 & 00037 & 00028 & 00025 & 00038\end{array}$

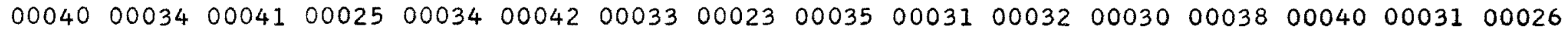
$\begin{array}{lllllllllllllllllllllll}0 & 00039 & 00028 & 00041 & 00035 & 00034 & 00040 & 00052 & 00037 & 00041 & 00041 & 00041 & 00021 & 00042 & 00081 & 00042\end{array}$ $00046 \quad 000340004500055 \quad 00045 \quad 0004700048 \quad 000450004200048 \quad 00036 \quad 0004100050 \quad 000670005100054$ $\begin{array}{llllllllllllllllll}00062 & 00050 & 00056 & 00064 & 00061 & 00059 & 00061 & 00064 & 00061 & 00077 & 00080 & 00053 & 00032 & 00029 & 00012 & 00016\end{array}$ 00014000080000600003000000000100000000000000000000000000000000000000050000005716

CRYSTAL HEIGHT $=06.000000$ IN. CRYSTAL RADIUS $=03.000000$ IN. $I=10000$ 000000000000000240002400029000210002400029000270001500026 $\begin{array}{llllllllllllllll} & 00028 & 00031 & 00024 & 00027 & 00028 & 00029 & 00027 & 00028 & 00021 & 00026\end{array}$ $\begin{array}{llllllllllllllll}00029 & 00031 & 00030 & 00027 & 00036 & 00026 & 00024 & 00028 & 00032 & 00037 & 00029 & 00028 & 00026 & 00040 & 00026 & 00032\end{array}$ $\begin{array}{lllllllllllllllll}00033 & 00036 & 00025 & 00034 & 00025 & 00039 & 00034 & 00027 & 00033 & 00031 & 00028 & 00021 & 00029 & 00029 & 00029 & 00028\end{array}$ $\begin{array}{lllllllllllllllll}00036 & 00038 & 00036 & 00034 & 00035 & 00044 & 00035 & 00043 & 00038 & 00046 & 00035 & 00047 & 00035 & 00033 & 00091 & 00042\end{array}$ $\begin{array}{lllllllllllllllll}0 & 00035 & 00052 & 00049 & 00041 & 00054 & 00047 & 00050 & 00062 & 00057 & 00062 & 00046 & 00048 & 00060 & 00059 & 00059 & 00048\end{array}$

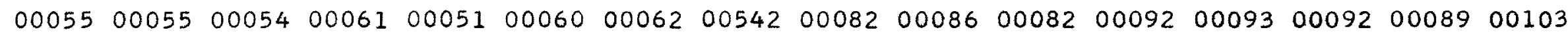
00116001250010400088000860003800035000170001200005000050000300003000050002604410

CRYSTAL HEIGHT $=06.000000$ IN. CRYSTAL RADIUS $=03.000000$ IN. $I=10000$

000110002400015000280001300022000200002000022 $00024000210002100024000240002300016 \quad 00018 \quad 0001900016 \quad 000170002300020000220002100025$

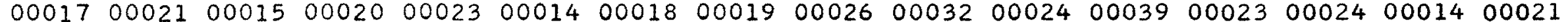

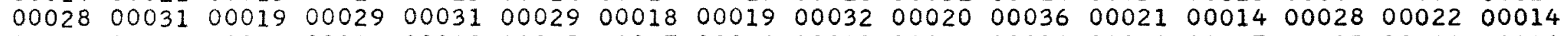
$\begin{array}{llllllllllllllll}00024 & 00021 & 00034 & 00013 & 00028 & 00025 & 00027 & 00026 & 00028 & 00034 & 00016 & 00032 & 00027 & 00025 & 00029 & 00026\end{array}$

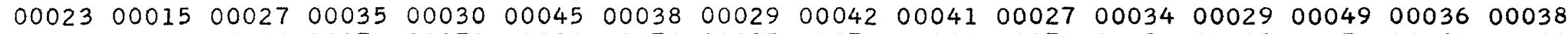
$0004100038 \quad 00166 \quad 00072 \quad 0007900080 \quad 00078 \quad 0008500071 \quad 00081000740008400068000700006400063$ 00081013720017200184001710016800148001560017400071000870003800020000040000403616 $\begin{array}{ll}\text { INCIDENT ENERGY } & =04.450000 \mathrm{MEV} \\ \text { SOURCE HEIGHT } & =00.000000\end{array}$ $P=04014$ INCIDENT ENERGY $=02.620000 \mathrm{MEV}$. SOURCE HEIGHT $=00.000000$ IN. $P=03339$

INCIDENT ENERGY $=01.330000 \mathrm{MEV} \cdot$ SOURCE HEIGHT $=00.000000$ IN. $P=02084$ 
TABLE I (CONTINUED)

CRYSTAL HEIGHT $=06.000000$ IN. CRYSTAL RADIUS $=03.000000$ IN. $I=10000$

$\begin{array}{lllllll}00020 & 00017 & 00019 & 00015 & 00025 & 00017 & 00021\end{array}$ $\begin{array}{lllllllll}00019 & 00013 & 00014 & 00018 & 00013 & 00014 & 00013 & 00 \\ 00022 & 00031 & 00017 & 00018 & 00021 & 00019 & 00016 & 0\end{array}$ 00019000190002200025000220002300027 $00027000280002500030 \quad 0002200030 \quad 00025$ 00035000250003500041000450004400033

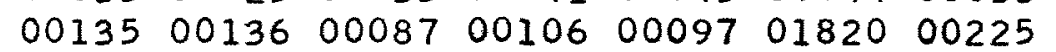

INCIDENT ENERGY $=06.130000 \mathrm{MEV}$. SOURCE HEIGHT $=00.000000$ IN. $P=04067$

$$
A=03223
$$
$\begin{array}{lllllllll}00015 & 00015 & 00015 & 00007 & 00016 & 00018 & 00024 & 00015 & 00016 \\ 00011 & 00020 & 00019 & 00015 & 00021 & 00017 & 00022 & 00029 & 00016\end{array}$ $\begin{array}{llllllllll}0 & 0013 & 00017 & 00012 & 00023 & 00009 & 00016 & 00012 & 00024 & 00018\end{array}$ $\begin{array}{llllllllll}0 & 0024 & 00017 & 00013 & 00013 & 00015 & 00017 & 00013 & 00020 & 00018\end{array}$

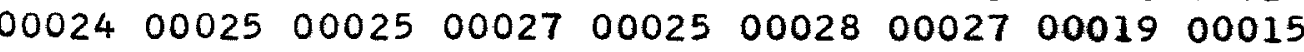
$\begin{array}{llllllllll}0 & 0023 & 00023 & 00027 & 00020 & 00027 & 00027 & 00031 & 00033 & 00037\end{array}$ $000440004400045002110010400105 \quad 00120 \quad 0011600093$ $\begin{array}{lllllllll}00238 & 00226 & 00271 & 00216 & 00112 & 00056 & 00011 & 00001 & 03235\end{array}$
CRYSTAL HEIGHT $=06.000000$ IN. CRYSTAL RADIUS $=03.000000$ IN. $I=10000$

$A=03002$

INCIDENT ENERGY $=07.100000 \mathrm{MEV}$. SOURCE HEIGHT $=00.000000$ IN. $00010 \quad 000130001400013000120001400012000130001200014000160001600017000240001200007$ 00011000160001100011000200001400019000150001300019000090001400014000150001000013

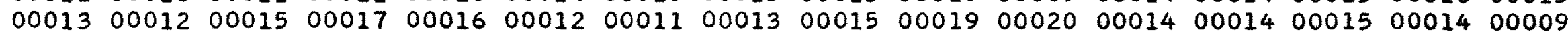
$000110001500016 \quad 000120001500018 \quad 00012000180001400026000200003100020000150001400015$ $\begin{array}{llllllllllllllll}00026 & 00027 & 00014 & 00017 & 00025 & 00016 & 00023 & 00015 & 00019 & 00023 & 00019 & 00019 & 00021 & 00015 & 00023 & 00020\end{array}$

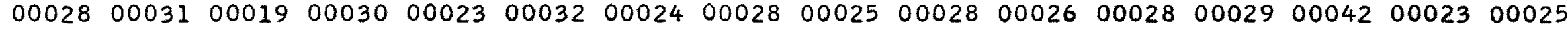
$00033000330002300026 \quad 0003200038 \quad 00044000440004100040 \quad 00060 \quad 0005400066 \quad 002530012900143$ $\begin{array}{llllllllllllllllll}00133 & 00139 & 00140 & 00109 & 00161 & 00088 & 01820 & 00312 & 00381 & 00281 & 00285 & 00215 & 00101 & 00051 & 00003 & 03015\end{array}$

CRYSTAL HEIGHT $=06.000000$ IN. CRYSTAL RADIUS $=03.000000 \mathrm{IN}$. $I=10000$ 008000000008000110001300016000110001400013000160001300014 $000100001100006 \quad 00014000160001400012000090001000015000140001100021000100001000011$ $00010 \quad 0000700016 \quad 0001100018 \quad 00015000140001200017000150002200018000070002100014 \quad 00018$

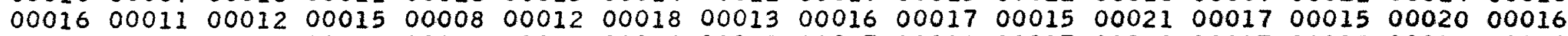
$00019000170001400016 \quad 00028 \quad 0002100016 \quad 000210002700014000170002000017000150001100028$

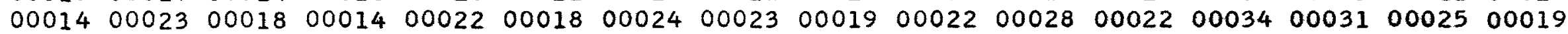

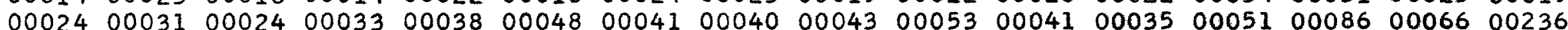

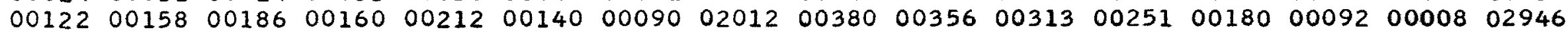
INCIDENT ENERGY $=08.000000$ MEV.
SOURCE HEIGHT $=00.000000$ IN. $A=02933 \quad$ SOURCE HEIGHT $=00.000000$ IN.
$P=03953$ 
CRYSTAL HEIGHT $=06.000000 \mathrm{IN}$ CRYSTAL RADIUS $=03.000000$ IN. $I=10000$ 00008000080000600009000110000700010000090001400007000150001000011000070000800009 $0001000006 \quad 0000500011000070001500014000110001200009000100001100010 \quad 000150000700011$ $00010 \quad 000140001200012000110001100018000120000800014000130001400011000100001500013$ $000110001400020 \quad 00010 \quad 00015 \quad 00015000140001900015000110001400009000130001200006 \quad 00017$ $0001100012000220002100016 \quad 0001300017000200001600009000110001700012000130002100021$

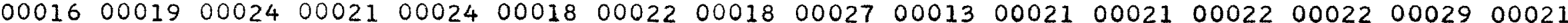
$0002900029000290003500032000340004500048 \quad 000450002900050 \quad 0004500048000510006300064$ $\begin{array}{lllllllllllllllll}00102 & 00117 & 00230 & 00220 & 00215 & 00198 & 00392 & 00224 & 00137 & 02118 & 00466 & 00292 & 00322 & 00223 & 00025 & 02727\end{array}$

CRYSTAL HEIGHT $=06.000000$ IN CRYSTAL RADIUS $=03.000000$ IN. $I=10000$

$0001100007000040000400010 \quad 0001000008000080001700009000090001400005000130001000011$ $00010 \quad 000070001100004000090001300014000140001500010000090001100009000070001100020$ $0000900009000120000900011 \quad 00008 \quad 00010 \quad 00011000120000900006 \quad 00013000120001500007 \quad 00012$ $0000600011000160000600013 \quad 0001000008 \quad 000110000900014000110001600016000110002100022$ $000130000900021 \quad 0001700011 \quad 0001900013 \quad 0001400017000170002000011000200001000018 \quad 00019$ $\begin{array}{lllllllllllllllll}00018 & 00016 & 00019 & 00025 & 00021 & 00022 & 00020 & 00025 & 00019 & 00010 & 00020 & 00023 & 00017 & 00022 & 00027 & 00034\end{array}$ $\begin{array}{llllllllllllllllll}00033 & 00037 & 00017 & 00033 & 00037 & 00034 & 00028 & 00028 & 00039 & 00031 & 00035 & 00050 & 00037 & 00050 & 00076 & 00081\end{array}$ $0011400086 \quad 00133001130020900356 \quad 0026500575003430022301913 \quad 00490 \quad 004850033300082 \quad 02357$

CRYSTAL HEIGHT $=06.000000$ IN. CRYSTAL RADIUS $=03.000000$ IN. $I=10000$ 00006000120000600010000080001100004000090000900011000050000500008000050000700011 $0000600008 \quad 0000800009000130001100008000080000700007000060000900009000090001100011$ $0000300012000070000700005000130001500009000150000900008000120000500006 \quad 0001100009$ 00009000050000500009000050001100014000140001400013000180001100011000150001700015 $0001200010 \quad 0001200012000120001400008 \quad 000100002000017000150001800014000130001800019$

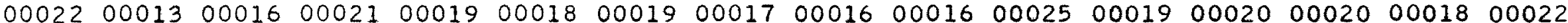

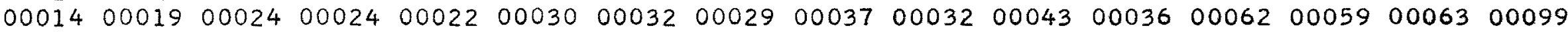
$\begin{array}{llllllllllllllllll}0 & 00102 & 00140 & 00201 & 00261 & 00265 & 00339 & 00630 & 00474 & 00440 & 00245 & 01663 & 00772 & 00399 & 00151 & 02057\end{array}$

INCIDENT ENERGY $=14.000000 \mathrm{MEV}$ SOURCE HEIGHT $=00.000000 \mathrm{IN}$. $P=03373$

INCIDENT ENERGY $=12.000000 \mathrm{MEV}$ SOURCE HEIGHT $=00.000000$ IN.

INCIDENT ENERGY $=10.000000 \mathrm{MEV}$. SOURCE HEIGHT $=00.000000$ IN. 4 
TABLE II

BROAD PARALLEL BEAM INCIDENT ON NaI CRYSTAL

CRYSTAL HEIGHT $=01.000000$ IN. CRYSTAL RADIUS $=00.500000 \mathrm{IN}$. $I=10000$

$A=07255$

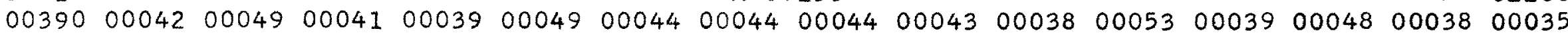
$\begin{array}{llllllllllllllll}00040 & 00036 & 00042 & 00028 & 00039 & 00035 & 00043 & 00028 & 00021 & 00033 & 00036 & 00046 & 00028 & 00037 & 00045 & 00027\end{array}$

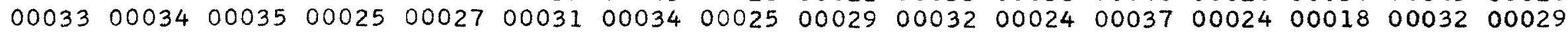
$000250002700030 \quad 00034 \quad 00016 \quad 00026 \quad 00032 \quad 00026 \quad 00030 \quad 000350002300036 \quad 00030 \quad 000240003100030$ $0003700041000290000600006 \quad 0000200007000000000300002000020000200002000010000100003$ $0000200000 \quad 00000 \quad 0000100000 \quad 00000 \quad 00000 \quad 000000000000000000000000000000000000000000000$ $00000 \quad 00000 \quad 00000 \quad 00000 \quad 00000 \quad 00000 \quad 00000 \quad 000000000000000000000000000000000000000000000$ 00000000000000000000000720000200000000000000000000000000000000000000000000007255
CRYSTAL HEIGHT $=01.000000$ IN. CRYSTAL RADIUS $=00.500000$ IN. $I=10000$

$\begin{array}{llllllll}00068 & 00064 & 00066 & 00051 & 00072 & 00076 & 00071\end{array}$

$0006800076 \quad 0006500067000690007200077$

$\begin{array}{lllllllll}00071 & 00070 & 00056 & 00073 & 00079 & 00071 & 00053 & 00 \\ 00065 & 00060 & 00057 & 00058 & 00064 & 00069 & 00078 & 006\end{array}$

$\begin{array}{llllllll}00065 & 00060 & 00057 & 00058 & 00064 & 00069 & 00078 \\ 00072 & 00082 & 00075 & 00057 & 00091 & 00092 & 00088\end{array}$

$0001800012000090000900006 \quad 00008 \quad 00002$

00000000000000000000000000000000000
$A=03116$

INCIDENT ENERGY $=00.661000 \mathrm{MEV}$ SOURCE HEIGHT

$=00.000000$ IN. $P=09393$
$0007500064000590006500073 \quad 00078 \quad 00083 \quad 00063 \quad 00081$ $\begin{array}{lllllllllll}00066 & 00054 & 00076 & 00056 & 00061 & 00068 & 00066 & 00082 & 00054\end{array}$ $00067000790006900070 \quad 0006200051000660004600080$ $\begin{array}{lllllllllll}00059 & 00060 & 00059 & 00069 & 00061 & 00068 & 00067 & 00063 & 00066\end{array}$ $\begin{array}{llllllllll}00068 & 00071 & 00074 & 00079 & 00086 & 00081 & 00082 & 00056 & 00075\end{array}$ $0007900090 \quad 0010100108 \quad 0008700041000190002000024$ 000060000500004000010000000000000010000000000 000000000000000000000003100000000000000003116
CRYSTAL HEIGHT $=01.000000$ IN. CRYSTAL RADIUS $=00.500000 \mathrm{IN}$. $I=10000$

001570005300051000570005800065000710007700069

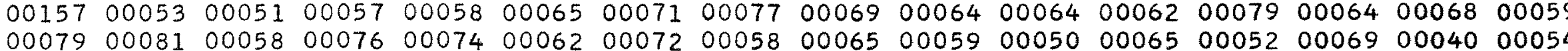
$0005100064000630007200066 \quad 00066 \quad 00083 \quad 0007900066 \quad 0009800078 \quad 00062000740005300056 \quad 00052$ $00056 \quad 00066 \quad 00058 \quad 00076 \quad 0006900073 \quad 0006900062 \quad 00063 \quad 00063 \quad 0006900072 \quad 000740007100074 \quad 00066$ 00072000800008300099000880007700088000890007100089000990011500090000990011000104 $\begin{array}{lllllllllllllllll}00099 & 00114 & 00122 & 00131 & 00139 & 00131 & 00125 & 00118 & 00135 & 00041 & 00033 & 00027 & 00027 & 00020 & 00016 & 00013\end{array}$ 00012000090000200002000000000000000000010000000000000000000100000000140000001962

INCIDENT ENERGY $=01.170000 \mathrm{MEV}$. SOURCE HEIGHT $=00.000000$ IN. $P=15342$ $\begin{array}{llllllllllllllll}00047 & 00071 & 00059 & 00060 & 00066 & 00039 & 00057 & 00057 & 00053 & 00059 & 00080 & 00064 & 00062 & 00060 & 00056 & 00067\end{array}$

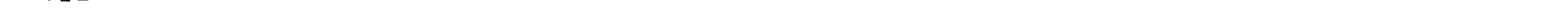


CRYSTAL HEIGHT $=01.000000$ IN. CRYSTAL RADIUS $=00.500000$ IN. $I=10000$

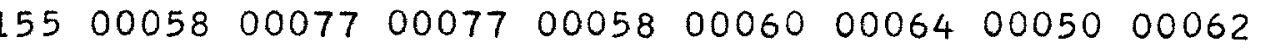

$0004500056 \quad 00062000600005100060 \quad 000680005300060$

000660006200063000540007200067000640005500057006

00069000610005700052000490007700075

$0007000071 \quad 00088000720007000090$

001110013100113001130014000126001

00023000090001000005000040000100000
INCIDENT ENERGY $=01.330000 \mathrm{MEV}$ SOURCE HEIGHT $=00.000000$ IN. $P=16720$ $\begin{array}{lllllll}00063 & 00065 & 00064 & 00059 & 00062 & 00055 & 00062\end{array}$ $\begin{array}{llllllll}0059 & 00059 & 00057 & 00059 & 00104 & 00049 & 00067 \\ 0058 & 00066 & 00061 & 00065 & 00054 & 00066 & 00053\end{array}$ $\begin{array}{llllllllll}00070 & 00075 & 00059 & 00066 & 00066 & 00054 & 00064 & 00064 & 00060 \\ 00068 & 00086 & 00058 & 00067 & 00069 & 00074 & 00073 & 00095 & 00062\end{array}$ $\begin{array}{lllllllllll}00068 & 00086 & 0 & 0 & 0 & 0 & 0 & 0 & 0 & 0 & 0\end{array}$ $\begin{array}{llllllllll}0 & 0136 & 00112 & 00161 & 00168 & 00091 & 00040 & 00034 & 00025 & 00011\end{array}$ 000000000100000000000000000000000180000101693

CRYSTAL HEIGHT $=01.000000$ IN. CRYSTAL RADIUS $=00.500000$ IN. $I=10000$ $0005400046 \quad 0004500051 \quad 00053 \quad 00056 \quad 0004900048 \quad 0006500050 \quad 0004300057 \quad 000580004800060 \quad 00059$ $\begin{array}{lllllllllllllllll}00051 & 00057 & 00046 & 00055 & 00052 & 00064 & 00046 & 00059 & 00052 & 00044 & 00056 & 00056 & 00047 & 00052 & 00063 & 00064\end{array}$ 00069000660004700059000610007200049000660006900057000550004800062000610005500062 $\begin{array}{llllllllllllllllll}00051 & 00051 & 00050 & 00056 & 00056 & 00028 & 00071 & 00063 & 00052 & 00038 & 00061 & 00058 & 00049 & 00050 & 00710 & 00082\end{array}$

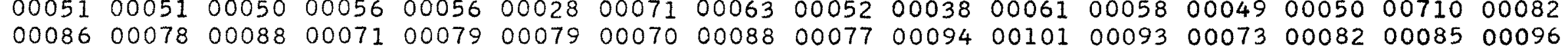
$\begin{array}{llllllllllllllll}00089 & 00082 & 00075 & 00088 & 00086 & 00088 & 00095 & 00304 & 00096 & 00096 & 00126 & 00126 & 00120 & 00119 & 00125 & 00135\end{array}$ 00154001510014500162001110005100030000190001200006000010001600004000040001000846

CRYSTAL HEIGHT $=01.000000$ IN. CRYSTAL RADIUS $=00.500000 \mathrm{IN}$. $I=10000$

000460005300059000440004100029000380003800039 000530004300032000520004900055 $00047000480004500040 \quad 00040 \quad 00055000$ 000440003900047000530004100040 $00050 \quad 0004000041000610004300058 \quad 004$ 000530003500057000640003800043

$0006100076 \quad 01547001450016100167$ 001050064800121001090011300126
INCIDENT ENERGY $=02.620000 \mathrm{MEV}$ SOURCE HEIGHT $=00.000000 \mathrm{IN}$. $P=23656$ $0006400052 \quad 00053 \quad 0004100060 \quad 00052 \quad 00047 \quad 00056 \quad 00068 \quad 00051$

INCIDENT ENERGY $=04.450000 \mathrm{MEV}$.

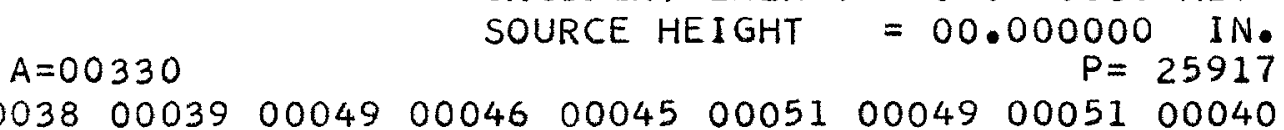

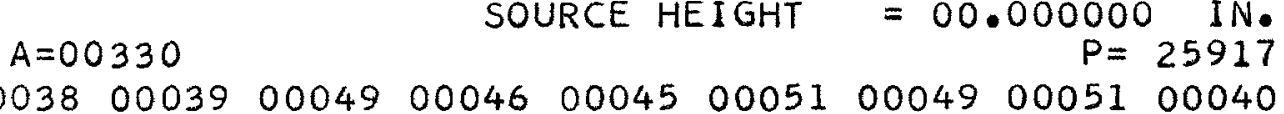
$0004500047 \quad 00050 \quad 0004700046 \quad 0004400046 \quad 00046 \quad 00051 \quad 00040$ $000440005400037 \quad 00048 \quad 00043 \quad 000490004500030 \quad 0004900053$ $\begin{array}{llllllllllll}00042 & 00038 & 00042 & 00044 & 00047 & 00050 & 00051 & 00055 & 00061 & 00055\end{array}$ $0004100047 \quad 0004400048 \quad 00057 \quad 0004400060000320005900043$ $\begin{array}{llllllllllll}00055 & 00053 & 00045 & 00050 & 00053 & 00054 & 00053 & 00043 & 00051 & 00051\end{array}$ $\begin{array}{lllllllllll}0 & 0135 & 00113 & 00131 & 00137 & 00137 & 00136 & 00125 & 00096 & 00089 & 00100\end{array}$ 00105001200014400059000920004100009000030000400339 
TABLE II (CONTINUED)

CRYSTAL HEIGHT $=01.000000$ IN. CRYSTAL RADIUS $=00.500000$ IN. $I=10000$ $\begin{array}{lllllllll} & 0\end{array}$ $0004700041000370004300041 \quad 00048 \quad 00049000410004400043000390004300040 \quad 0002700042 \quad 00044$ $\begin{array}{llllllllllllllll}00035 & 00035 & 00044 & 00038 & 00028 & 00039 & 00038 & 00048 & 00047 & 00039 & 00047 & 00035 & 00040 & 00043 & 00044 & 00036\end{array}$ $\begin{array}{llllllllllllllll}00046 & 00032 & 00053 & 00031 & 00053 & 00045 & 00047 & 00051 & 00045 & 00034 & 00042 & 00041 & 00046 & 00039 & 00055 & 00036\end{array}$ $\begin{array}{llllllllllllllllll}0 & 0034 & 00038 & 00037 & 00039 & 00052 & 00033 & 00043 & 00045 & 00043 & 00053 & 00036 & 00040 & 00050 & 00044 & 00051 & 00042\end{array}$

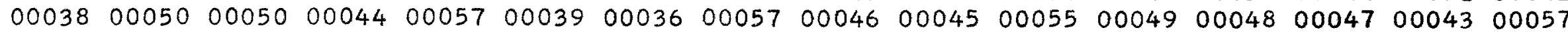
$\begin{array}{llllllllllllllllll}0 & 0064 & 00047 & 00045 & 00051 & 00057 & 00054 & 00070 & 00099 & 00186 & 00073 & 01947 & 00215 & 00193 & 00238 & 00244 & 00198\end{array}$

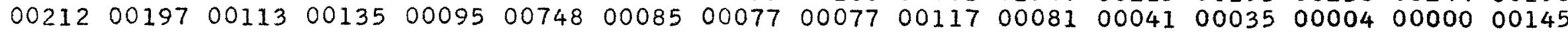

CRYSTAL HEIGHT $=01.000000$ IN.

CRYSTAL RADIUS $=00.500000 \mathrm{IN}$. $I=10000$

$0002900046 \quad 00046 \quad 0004700045 \quad 000410003400050 \quad 00036 \quad 00037000270005100035000400003100038$

$\begin{array}{llllllllllllllll}00039 & 00039 & 00039 & 00040 & 00036 & 00034 & 00043 & 00043 & 00026 & 00044 & 00047 & 00042 & 00036 & 00053 & 00039 & 00043\end{array}$

$000260004900033 \quad 0004400045 \quad 00043 \quad 00038 \quad 00050 \quad 000450004900056 \quad 00040 \quad 0005700046 \quad 00038 \quad 00049$

$0004400050 \quad 00033000320004300041000320004700044000330005100041000520005200050 \quad 00036$

$00058 \quad 00045 \quad 0003700048 \quad 00046 \quad 00033 \quad 000290004500043000330005500053 \quad 000450004700046 \quad 00031$

$\begin{array}{lllllllllllllllll}00049 & 00043 & 00042 & 00048 & 00063 & 00046 & 00060 & 00072 & 00147 & 00072 & 00084 & 00456 & 00124 & 01853 & 00277 & 00262\end{array}$ 00241002360020700201001880006200702000860007000056000560003200030000250000300091

CRYSTAL HEIGHT $=01.000000$ IN. CRYSTAL RADIUS $=00.500000$ IN. $I=10000$

$0005200030 \quad 0002800029000340002800048 \quad 00032000360003800030 \quad 0005400049000320004300043$ $\begin{array}{lllllllllllllllll}00046 & 00032 & 00026 & 00035 & 00036 & 00033 & 00035 & 00050 & 00039 & 00036 & 00032 & 00037 & 00039 & 00041 & 00032 & 00044\end{array}$ $\begin{array}{llllllllllllllll}00040 & 00052 & 00035 & 00046 & 00033 & 00047 & 00038 & 00041 & 00044 & 00039 & 00037 & 00054 & 00032 & 00036 & 00036 & 00040\end{array}$ $000520004200040 \quad 00033 \quad 00038 \quad 00038 \quad 0003500042000420004500030 \quad 0004200048 \quad 0004100048 \quad 00041$ $0004300043 \quad 0004500042000470004900057000450004700059000450005000047000500004100049$ $\begin{array}{lllllllllllllllll}0 & 0036 & 00047 & 00051 & 00041 & 00039 & 00049 & 00048 & 00050 & 00046 & 00054 & 00055 & 00037 & 00055 & 00040 & 00048 & 00054\end{array}$ $000550004700051000600006200070 \quad 00055000620006200096001190010100424003430013101706$ $0029200270 \quad 0028000195002910016400050 \quad 0067000060 \quad 00053000430003700030000200000100085$ $\begin{array}{ll}\text { INCIDENT ENERGY } & =08.000000 \text { MEV } \\ \text { SOURCE HEIGHT } & =00.000000 \text { IN. }\end{array}$ $P=25394$ INCIDENT ENERGY $=07.100000$ MEV. SOURCE HEIGHT $=00.000000$ IN. (1) 
CRYSTAL HEIGHT $=02.000000$ IN. CRYSTAL RADIUS $=01.000000$ IN. $I=10000$

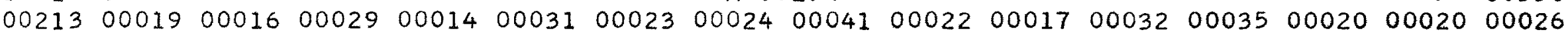
$00016 \quad 00027 \quad 00016 \quad 00022 \quad 00016 \quad 00023 \quad 00028 \quad 000250002500021000200002100021000220001900014$

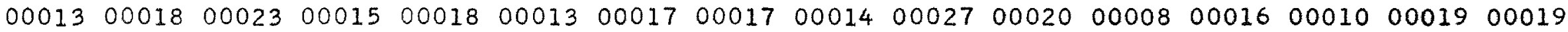
$\begin{array}{llllllllllllllll}00018 & 00015 & 00018 & 00023 & 00018 & 00014 & 00021 & 00018 & 00026 & 00018 & 00022 & 00029 & 00017 & 00028 & 00033 & 00030\end{array}$ 00027000270002900003000050000400001000030000000002000050000200000000000000100000 $0000000000 \quad 00000 \quad 00000 \quad 00000 \quad 00000 \quad 00000 \quad 00000 \quad 0000000000000010000000000 \quad 0000000000 \quad 00000$ $000000000000000 \quad 0000000000 \quad 00000 \quad 00000 \quad 000000000000000000000000000000 \quad 0000000000 \quad 00000$ 00000000000000000000000590000400000000000000000000000000000000000000000000008294

CRYSTAL HEIGHT $=02.000000$ IN. CRYSTAL RADIUS $=01.000000$ IN. $I=10000$ $18700049000480005800045000460005700060 \quad 00034$ $0005100057000550004300043 \quad 0004400040 \quad 000540003700054000510006500075000360004900047$ $0004400062 \quad 00051 \quad 00055 \quad 00051 \quad 00055 \quad 00067 \quad 00045000620004400053 \quad 00050 \quad 00053 \quad 0004700048 \quad 00050$ $0006400053 \quad 0005900049000690004300051 \quad 00040 \quad 000470004100046000410006400050 \quad 00050 \quad 00057$ $\begin{array}{lllllllllllllllllllll}00058 & 00049 & 00057 & 00046 & 00061 & 00061 & 00061 & 00058 & 00055 & 00072 & 00060 & 00057 & 00058 & 00061 & 00061 & 00058\end{array}$ $00058 \quad 0006600058 \quad 000620005900073 \quad 00071 \quad 000660007200080 \quad 000690006500037000200002000009$ $0001000008 \quad 00006 \quad 0000700010 \quad 00003000040000400001000020000100000000000000000000 \quad 00000$ 00000000000000000000000000000000000000000000000000000000001800000000000000004704

CRYSTAL HEIGHT $=02.000000$ IN. CRYSTAL RADIUS $=01.000000$ IN. $I=10000$

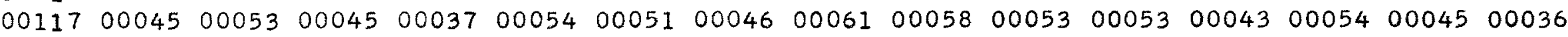
$\begin{array}{lllllllllllllllll}00065 & 00052 & 00056 & 00047 & 00052 & 00059 & 00059 & 00052 & 00059 & 00050 & 00056 & 00047 & 00043 & 00062 & 00053 & 00046\end{array}$ $0004600048 \quad 00066 \quad 0005200052 \quad 00062000590006300066000440006300050 \quad 0005500053 \quad 0005900051$ $\begin{array}{llllllllllllllllllll}00050 & 00038 & 00061 & 00061 & 00046 & 00051 & 00034 & 00056 & 00045 & 00057 & 00049 & 00065 & 00053 & 00050 & 00057 & 00049\end{array}$ $00050 \quad 000570005300055000590006500066 \quad 00074000880006200066 \quad 0006800059000630007400061$ $\begin{array}{lllllllllllllllllll}0 & 0 & 0 & 0 & 077 & 00064 & 00081 & 00069 & 00072 & 00074 & 00065 & 00084 & 00074 & 00089 & 00078 & 00080 & 00081 & 00089 & 00103\end{array}$ $00116000850010100100 \quad 00090 \quad 00100 \quad 00091000970010500029000260002100022000170001200012$ 00012000010000000002000010000000002000010000000000000000000000000000110000003253 
TABLE II (CONTINUED)

CRYSTAL HEIGHT $=02.000000$ IN. CRYSTAL RADIUS $=01.000000 \mathrm{IN}$. $I=10000$

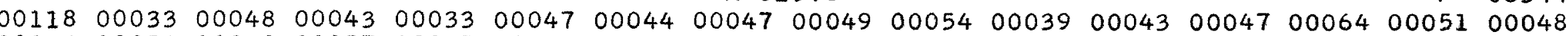
$\begin{array}{lllllllllllllllll}00046 & 00059 & 00040 & 00057 & 00037 & 00055 & 00035 & 00046 & 00046 & 00038 & 00052 & 00048 & 00051 & 00083 & 00051 & 00049\end{array}$ $0005100034000510003800044000620005500050 \quad 0005000060000510005200046000470007200058$ $\begin{array}{lllllllllllllllll}0 & 0049 & 00048 & 00051 & 00056 & 00047 & 00061 & 00066 & 00062 & 00050 & 00061 & 00067 & 00054 & 00061 & 00075 & 00077 & 00041\end{array}$ $\begin{array}{llllllllllllllll}00067 & 00063 & 00061 & 00040 & 00052 & 00060 & 00063 & 00057 & 00057 & 00065 & 00070 & 00068 & 00059 & 00074 & 00096 & 00067\end{array}$ $\begin{array}{lllllllllllllllll}00060 & 00066 & 00078 & 00066 & 00059 & 00071 & 00083 & 00079 & 00081 & 00075 & 00083 & 00089 & 00080 & 00083 & 00092 & 00082\end{array}$ $000930011000084001140011500099001050012400105001260011200061000390002400024 \quad 00022$ 00013000110001000010000040000100000000000000000000000000000000000000210000102978

CRYSTAL HEIGHT $=02.000000$ IN. CRYSTAL RADIUS $=01.000000$ IN. $I=10000$ $000630003800040000370003500040 \quad 000330003300045$ $000590004700038 \quad 00036 \quad 0003700036 \quad 00040 \quad 0003200046 \quad 00036 \quad 0004700044000450004500036 \quad 00033$ $\begin{array}{lllllllllllllllllll}00048 & 00039 & 00036 & 00048 & 00040 & 00044 & 00058 & 00041 & 00039 & 00056 & 00040 & 00031 & 00043 & 00043 & 00040 & 00051\end{array}$ $00033000490003200050 \quad 00047000390005400041000490005100058 \quad 0005400043000350004700051$ $\begin{array}{lllllllllllllllll}00040 & 00053 & 00044 & 00067 & 00052 & 00048 & 00048 & 00058 & 00042 & 00062 & 00039 & 00055 & 00052 & 00054 & 00420 & 00070\end{array}$

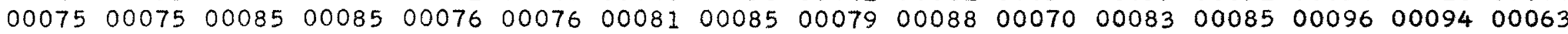
$\begin{array}{lllllllllllllllll}00084 & 00084 & 00074 & 00088 & 00087 & 00087 & 00076 & 00481 & 00097 & 00098 & 00111 & 00123 & 00101 & 00135 & 00142 & 00154\end{array}$ $0014700145001420014500108 \quad 00053 \quad 000470002200016000040000300006 \quad 00003000050001401893$

CRYSTAL HEIGHT $=02.000000$ IN. CRYSTAL RADIUS $=01.000000$ IN. $I=10000$

$000230004100027000230002500026 \quad 00036 \quad 000310002800034000270002700022000330003300032$ $\begin{array}{llllllllllllllllll}00025 & 00033 & 00035 & 00033 & 00035 & 00037 & 00036 & 00026 & 00042 & 00030 & 00026 & 00034 & 00036 & 00038 & 00046 & 00026\end{array}$

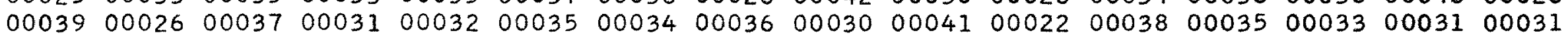
$\begin{array}{llllllllllllllllll}00035 & 00034 & 00042 & 00046 & 00033 & 00045 & 00040 & 00037 & 00033 & 00044 & 00034 & 00032 & 00043 & 00033 & 00037 & 00034\end{array}$ $000340002700040 \quad 000290002700038000380004400050 \quad 000410004600031000410003500050 \quad 00041$ $\begin{array}{lllllllllllllllll}00045 & 00049 & 00027 & 00048 & 00047 & 00046 & 00048 & 00037 & 00043 & 00043 & 00055 & 00070 & 00043 & 00058 & 00055 & 00055\end{array}$ $00054000670093400156 \quad 00150 \quad 0015900140 \quad 00150 \quad 00124001380015300137000950010200100 \quad 00099$ $000930108900180001450016300158001690016800173000810009500067000180000300006 \quad 01114$
INCIDENT ENERGY $=04.450000 \mathrm{MEV}$ SOURCE HEIGHT $=00.000000 \mathrm{IN}$. $P=10954$

INCIDENT ENERGY $=02.620000 \mathrm{MEV}$. SOURCE HEIGHT $=00.000000$ IN. $P=09611$ 
CRYSTAL HEIGHT $=02.000000$ IN. CRYSTAL RADIUS $=01.000000$ IN. $I=10000$

$000270001800024000280003300020 \quad 00023000310002600030000220002100030000310001300026$ $\begin{array}{lllllllllllllllllll}00021 & 00034 & 00031 & 00022 & 00028 & 00030 & 00025 & 00033 & 00031 & 00039 & 00035 & 00031 & 00029 & 00042 & 00034 & 00032\end{array}$ $\begin{array}{lllllllllllllllll}00025 & 00028 & 00022 & 00033 & 00032 & 00035 & 00027 & 00020 & 00022 & 00033 & 00024 & 00030 & 00027 & 00022 & 00038 & 00031\end{array}$ $\begin{array}{llllllllllllllll}00043 & 00017 & 00023 & 00032 & 00022 & 00027 & 00027 & 00034 & 00026 & 00030 & 00031 & 00029 & 00040 & 00035 & 00030 & 00035\end{array}$ $\begin{array}{llllllllllllllll}00037 & 00034 & 00036 & 00036 & 00027 & 00033 & 00023 & 00040 & 00032 & 00032 & 00037 & 00025 & 00030 & 00029 & 00025 & 00027\end{array}$

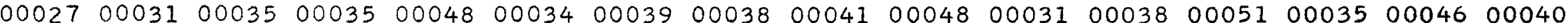
$0004400037 \quad 00058 \quad 000370006600051 \quad 00058 \quad 00076 \quad 00108 \quad 00061012640024700241002340023100212$

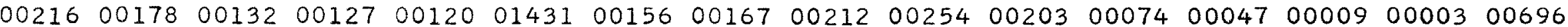

CRYSTAL HEIGHT $=02.000000$ IN. CRYSTAL RADIUS $=01.000000 \mathrm{IN}$. $I=10000$ $\begin{array}{lllllllllllllllll}00019 & 00021 & 00024 & 00032 & 00030 & 00038 & 00024 & 00028 & 00035 & 00022 & 00024 & 00033 & 00023 & 00022 & 00027 & 00028\end{array}$

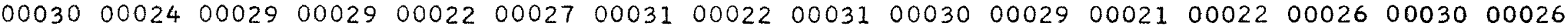
$\begin{array}{llllllllllllllllll}00023 & 00035 & 00023 & 00027 & 00017 & 00023 & 00024 & 00034 & 00029 & 00026 & 00024 & 00032 & 00023 & 00027 & 00027 & 00047\end{array}$ $\begin{array}{lllllllllllllllllll}00026 & 00042 & 00030 & 00033 & 00032 & 00035 & 00024 & 00040 & 00031 & 00023 & 00030 & 00026 & 00032 & 00037 & 00033 & 00023\end{array}$ $0004100032 \quad 00024 \quad 00029000410003400052 \quad 000370003200048 \quad 0003900036 \quad 00040000450004400041$ $00044000340004900038 \quad 00046 \quad 00065 \quad 00048 \quad 0004700086 \quad 000510008800216 \quad 000870122900315 \quad 00310$ 00301002610025300209002220009401459001960022600196001620014100080000310000000568

CRYSTAL HEIGHT $=02.000000$ IN. CRYSTAL RADIUS $=01.000000$ IN. $I=10000$ $0002100027 \quad 00016 \quad 00021 \quad 00021 \quad 00020 \quad 00027 \quad 00026 \quad 00023000170001700028000200001700025 \quad 00029$ $000290002400022 \quad 00024 \quad 00030 \quad 00035 \quad 00016 \quad 0002900026000310003100032 \quad 00034 \quad 000250002900024$

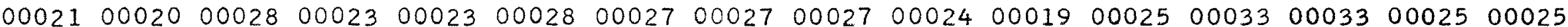

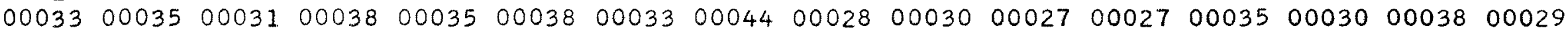
$00030 \quad 00038 \quad 00033 \quad 00026 \quad 00034 \quad 00025 \quad 00025 \quad 000330003700032000470003000033000350003100043$ $0004200036 \quad 00047 \quad 00040 \quad 00043 \quad 00045 \quad 00057 \quad 00049000420006300078 \quad 0008700219001780011101373$

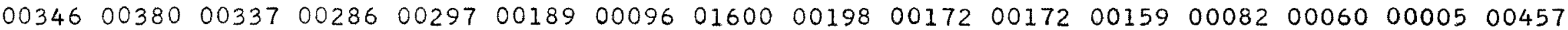

INCIDENT ENERGY $=08.000000 \mathrm{MEV}$. SOURCE HEIGHT $=00.000000$ IN. $P=10675$ INCIDENT ENERGY $=07.100000 \mathrm{MEV}$ SOURCE HEIGHT $=00.000000$ IN. $P=11220$

INCIDENT ENERGY $=06.130000 \mathrm{MEV}$. SOURCE HEIGHT $=00.000000 \mathrm{IN}$. $P=10872$ 
CRYSTAL HEIGHT $=04.000000 \mathrm{IN}$. CRYSTAL RADIUS $=02.000000 \mathrm{IN}$. $I=10000$

$001190000500006 \quad 000080000800014000100000900008$ $\begin{array}{llllllll}00015 & 00008 & 00007 & 00013 & 00008 & 00009 & 00009 \\ 00012 & 00007 & 00015 & 00009 & 00015 & 00013 & 00012\end{array}$ 00029000280002500001000020000300005 00000000000000100000000000000100000 00000000000000000000000000000000000 00000000000000000000000350000200000
INCIDENT ENERGY $=00.279000 \mathrm{MEV} \bullet$ SOURCE HEI GHT $=00.000000 \mathrm{IN}$ $P=00016$ $\begin{array}{llllllllll} & 00000 & 00008 & 00015 & 00010 & 00012 & 00012 & 00011\end{array}$ $0000600008 \quad 00013000120001800009000090000400013$ $00007 \quad 000120001300008 \quad 0001000011000050000800002$ 000190001800019000170002200021000230002000035 000020000100001000040000100001000000000100001 $000000000000000 \quad 0000000000000000000000000 \quad 00000$ 000000000000000000000000000000000000000000000 000000000000000000000000000000000000000009019
CRYSTAL HEIGHT $=04.000000$ IN. CRYSTAL RADIUS $=02.000000 \mathrm{IN}$. $I=10000$

$\begin{array}{lllllll}00115 & 00023 & 00024 & 00017 & 00024 & 00027 & 00019\end{array}$ $00030 \quad 00027 \quad 0003200025000290002900039$ $\begin{array}{llllllll}00035 & 00035 & 00022 & 00035 & 00039 & 00035 & 00035 \\ 00038 & 00029 & 00041 & 00037 & 00027 & 00035 & 00040\end{array}$ $00030 \quad 00036 \quad 000250003900030 \quad 0004100037$ $000540004900060 \quad 00045000470005900039$ $\begin{array}{llllllll}00012 & 00002 & 00008 & 00005 & 00002 & 00007 & 00003 \\ 00000 & 00000 & 00000 & 00000 & 00000 & 00000 & 00000\end{array}$
INCIDENT ENERGY $=00.661000 \mathrm{MEV}$. SOURCE HEIGHT $=00.000000$ IN. $A=06515$ $P=00588$ $0003700026 \quad 0002900034 \quad 0003100032000310002400039$ $\begin{array}{llllllllll}0 & 0046 & 00029 & 00041 & 00025 & 00042 & 00032 & 00028 & 00022 & 00033\end{array}$ $\begin{array}{lllllllllll}00041 & 00051 & 00043 & 00035 & 00032 & 00039 & 00039 & 00030 & 00035\end{array}$ $\begin{array}{llllllllll}00029 & 00025 & 00034 & 00040 & 00030 & 00038 & 00038 & 00034 & 00040\end{array}$ $00038 \quad 00023 \quad 00041 \quad 00023 \quad 0004400034000390004400036$ $0006300056 \quad 00057000530005500018000140001100012$ 000010000000000000000000000000000010000100000 000000000000000000000001400000000000000006515
CRYSTAL HEIGHT $=04.000000$ IN. CRYSTAL RADIUS $=02.000000 \mathrm{IN}$. $I=10000$

$A=05175$

INCIDENT ENERGY $=01.170000 \mathrm{MEV}$ SOURCE HEIGHT

$0008800029000390002300030 \quad 00026 \quad 000390002900033000380003200027000300003300026 \quad 00040$ $\begin{array}{llllllllllllllllll}00036 & 00027 & 00029 & 00034 & 00031 & 00026 & 00025 & 00037 & 00031 & 00035 & 00038 & 00043 & 00034 & 00034 & 00037 & 00033\end{array}$ $00030 \quad 00042 \quad 00030 \quad 00028 \quad 00032 \quad 00046 \quad 00033 \quad 00027 \quad 0002900050 \quad 0003400035000370003300036 \quad 00032$ $\begin{array}{llllllllllllllllll}00036 & 00050 & 00038 & 00038 & 00038 & 00048 & 00043 & 00055 & 00031 & 00034 & 00049 & 00050 & 00051 & 00039 & 00037 & 00045\end{array}$ $000410004700033000470004100039000450003800064000490005400044000410006600056 \quad 00042$ $\begin{array}{lllllllllllllllll}00051 & 00056 & 00058 & 00066 & 00054 & 00047 & 00053 & 00065 & 00073 & 00069 & 00056 & 00072 & 00066 & 00070 & 00082 & 00059\end{array}$ $0006800059000690006600080 \quad 00053 \quad 000790007600065000330001700020000220001000008 \quad 00009$ 00004000020000200001000000000000000000000000000000000000000100001000080000005175 
TABLE II (CONTINUED)

CRYSTAL HEIGHT $=04.000000$ IN. CRYSTAL RADIUS $=02.000000$ IN. $I=10000$

$000910002600030 \quad 000250002500020 \quad 00030 \quad 00040 \quad 00034000310003700043000410002400038 \quad 00034$

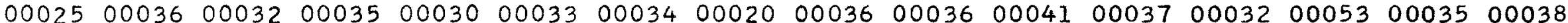
$0004100039000270003500035000410003900030 \quad 0002100040 \quad 0003200040 \quad 00033000410003400044$ $\begin{array}{llllllllllllllllll}00039 & 00039 & 00036 & 00031 & 00028 & 00046 & 00051 & 00045 & 00032 & 00049 & 00043 & 00045 & 00049 & 00045 & 00052 & 00047\end{array}$ $000340004400045000440004900046 \quad 00046 \quad 000420004700061000540004500040000510009900055$ $0004500056 \quad 00056 \quad 0004200042000620006700054000620007200057 \quad 00052 \quad 000590006300072 \quad 00079$ $\begin{array}{lllllllllllllllll}00064 & 00068 & 00069 & 00080 & 00081 & 00073 & 00094 & 00083 & 00076 & 00084 & 00071 & 00048 & 00033 & 00024 & 00017 & 00014\end{array}$ 00006000070000500003000020000100000000000000000000000000000000000000060000004878

CRYSTAL HEIGHT $=04.000000$ IN. CRYSTAL RADIUS $=02.000000 \mathrm{IN}$. $I=10000$

000240001500029000230002400029000220002300035 000340003300031000340003300023 000320003000028000310003700037 000270005000023000380003400033 $\begin{array}{llllll}00032 & 00030 & 00038 & 00045 & 00039 & 00043 \\ 00070 & 00060 & 00071 & 00070 & 00063 & 00054\end{array}$ $\begin{array}{llllll}00070 & 00060 & 00071 & 00070 & 00063 & 00054 \\ 00059 & 00058 & 00070 & 00070 & 00066 & 00061\end{array}$ 001260013500145001280010700051 000270002400040 00031000250002900047 0003500043000370004300042 0006400066000570005700067 0006700548001040012200093 0002300022000110000400002
INCIDENT ENERGY $=02.620000 \mathrm{MEV} \bullet$ SOURCE HEIGHT $=00.000000$ IN. $P=03135$ $0003000030 \quad 00026000270002500029$ $\begin{array}{llllllll}04 & 00024 & 00027 & 00030 & 00035 & 00029\end{array}$ $\begin{array}{llllll}00028 & 00031 & 00026 & 00025 & 00037\end{array}$ $\begin{array}{llllll}0 & 00035 & 00030 & 00032 & 00040 & 00033\end{array}$ $\begin{array}{llllll}0 & 00046 & 00042 & 00039 & 00171 & 00054\end{array}$ $\begin{array}{llllll}00071 & 00068 & 00088 & 00078 & 00059\end{array}$ $00106 \quad 00103000930010200132$ 0000300001000030001103586
CRYSTAL HEIGHT $=04.000000$ IN. CRYSTAL RADIUS $=02.000000$ IN. $I=10000$

000270001400021000150001200023000210001900017

$0002100018 \quad 00016 \quad 00016 \quad 00017000230001800019000240002000013 \quad 00028000170002300024 \quad 00023$ $000270001900014000200001700030 \quad 00023000270002700028000220002700032000240003200017$

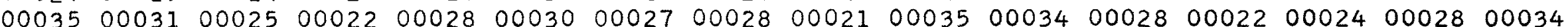

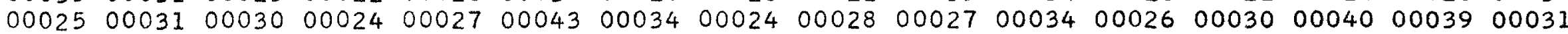
$00033 \quad 00032000330003400040 \quad 00033 \quad 00036 \quad 00032 \quad 0003200035000430004900046 \quad 000440004100049$ $000320004700406 \quad 00093 \quad 0011500103 \quad 001120010000120001070010200117000900008000070 \quad 00098$ 00082013620017700167001790016200181001840016200097000890004300015000040000302739

INCIDENT ENERGY $=04.450000$ MEV. SOURCE HEIGHT $=00.000000$ IN. $P=03773$ 000820136200177001670017900162 
TABLE II (CONTINUED)

CRYSTAL HEIGHT $=04.000000$ IN. CRYSTAL RADIUS $=02.000000$ IN. $I=10000$

000150000900016000190000700018000190001200016000150001100016000260000900008 00015000160001900023000210002000023000150001800022000220001500009000200001100021 00018000160002000014000190003000017000180001800017000210002200019000270002200012 $0001100023000120001400013 \quad 0002300020000220002300017000300001800018000310001500036$ $00027000270002200027 \quad 000240002100020 \quad 0002000022000130002900025000280002500030 \quad 00023$ $\begin{array}{lllllllllllllllll}00030 & 00027 & 00030 & 00023 & 00030 & 00030 & 00029 & 00022 & 00033 & 00035 & 00027 & 00024 & 00037 & 00029 & 00032 & 00036\end{array}$ 00037000300003700040000520004400039000480005800052004920016600139001580016700162 00155001650009200113001130180300302002550027500304002430012900064000080000202246

CRYSTAL HEIGHT $=04.000000$ IN. CRYSTAL RADIUS $=02.000000 \mathrm{IN}$ $I=10000$

00017000120001200011000170001400009000100001600011000130001100018000110001700016 00009000160001000011000080001600014000140001200014000110002000021000180001400027 $0001400012 \quad 000230002300020 \quad 00022000120001500021000230002200014000140001800026 \quad 00015$ 00007000110001900019000160001100010000130002000024000210001400020000180002200025 $00021000200002100026000240002200028000200002600016 \quad 0002300020000190003100030 \quad 00031$ $\begin{array}{lllllllllllllllll}00030 & 00021 & 00026 & 00021 & 00027 & 00023 & 00028 & 00031 & 00036 & 00026 & 00029 & 00022 & 00022 & 00027 & 00024 & 00031\end{array}$ $00030 \quad 00029000320003600036 \quad 0003900035000330004400041000450007300053 \quad 0056500196 \quad 00186$ $0016400187001800015900186001220197900340 \quad 003610032700300 \quad 0021400108000410000202041$

CRYSTAL HEIGHT $=04.000000$ IN. CRYSTAL RADIUS $=02.000000 \mathrm{IN}$. $I=10000$

000150001000011000140001400014000140001200013

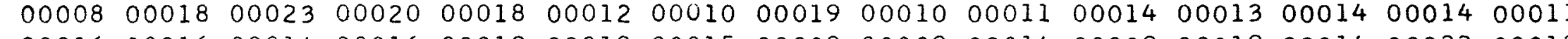
$000110001600016 \quad 0001400016 \quad 0001200019000150000900009000140000900019000140002200012$ 00015000130001900015000140001300014000150001900015000150001500019000250001900016 $00026 \quad 00020 \quad 00016 \quad 0002500011 \quad 0002300020 \quad 000270002000024000210002000020000130002700021$ 00030000190001900019000330002900024000250003100034000250003700027000240003000044 $\begin{array}{llllllllllllllll}00032 & 00023 & 00029 & 00041 & 00029 & 00034 & 00031 & 00049 & 00056 & 00057 & 00056 & 00048 & 00075 & 00078 & 00069 & 00619\end{array}$

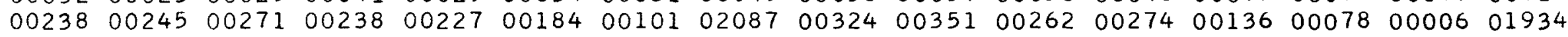

INCIDENT ENERGY $=08.000000 \mathrm{MEV}$. SOURCE HEIGHT $=00.000000$ IN. $P=02374$ 
CRYSTAL HEIGHT $=06.000000$ IN. CRYSTAL RADIUS $=03.000000$ IN. $I=10000$

000020000400004000090000500011000020000600005000090000400005000030000900004 $0000800006 \quad 00010 \quad 00015000090001400008 \quad 00006 \quad 0000500008 \quad 000070001400008000090001000010$ $000020000700007000030000500006 \quad 000100000900012000060000700006 \quad 00005000040000700009$ 00015000090001100005000050000700013000070001000016000200001800012000220002600021 00026000350001700004000030000600002000000000000001000000000000000000010000000001 $0000000000 \quad 00000 \quad 000000000000000 \quad 00000 \quad 000000000000000000000000000000000000000000000$ $0000000000 \quad 00000 \quad 00000 \quad 0000000000 \quad 00000 \quad 00000 \quad 000000000000000000000000000000 \quad 0000000000$ 00000000000000000000000150000200000000000000000000000000000000000000000000009254

CRYSTAL HEIGHT $=06.000000 \mathrm{IN}$. CRYSTAL RADIUS $=03.000000$ IN. $I=10000$ $0008400012000120001300010 \quad 00010000180001800013000140001000022000130001500010 \quad 00018$ 00023000170000900014000260001500017000230002000022000120002700025000160002600020

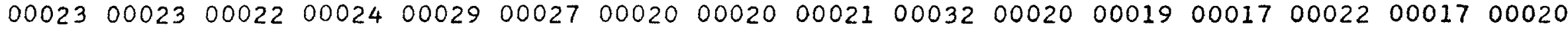
$0001100021000250002200018 \quad 00027 \quad 00026 \quad 0002600022000260002300019000340002500026 \quad 00018$ $\begin{array}{llllllllllllllllll}00031 & 00026 & 00026 & 00031 & 00030 & 00020 & 00029 & 00027 & 00025 & 00033 & 00033 & 00032 & 00030 & 00038 & 00038 & 00032\end{array}$

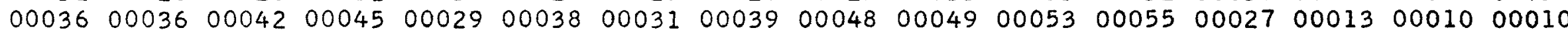
00005000040000600002000030000400002000000000100001000000000000000000000000000000 00000000000000000000000000000000000000000000000000000000001600000000000000007585

CRYSTAL HEIGHT $=06.000000$ IN. CRYSTAL RADIUS $=03.000000$ IN. $I=10000$

$A=06392$

00054000200001100014000150001200020000180001000019000230002200016000200002700021 $000290002200018 \quad 00027 \quad 0001900020 \quad 0002900027 \quad 00026 \quad 00022 \quad 00023 \quad 00030 \quad 0001500030 \quad 00025 \quad 00021$

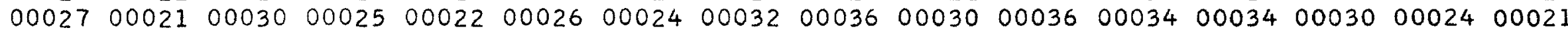
$\begin{array}{lllllllllllllllll}00033 & 00026 & 00020 & 00017 & 00026 & 00025 & 00033 & 00027 & 00025 & 00034 & 00035 & 00037 & 00038 & 00028 & 00034 & 00026\end{array}$ $0003200030 \quad 000350003900050 \quad 0004200044000310005700046000340002800042000380003200035$ $000360002900046000390003600049000420005700049000460005100041000510005500040 \quad 00050$ 00064000590005200044000580005900071000650006800024000270001800006000110000300007 00002000060000100001000000000000000000000000000001000000000000000000080000006392

INCIDENT ENERGY $=01.170000 \mathrm{MEV}$ SOURCE HEIGHT = 00.000000 IN. $P=00473$ 
CRYSTAL HEIGHT $=06.000000$ IN. CRYSTAL RADIUS $=03.000000$ IN. $I=10000$

$\begin{array}{llllllllllllllll}0 & 00011 & 00018 & 00011 & 00016 & 00018 & 00017 & 00016 & 00013 & 00020 & 00020 & 00016 & 00014 & 00018 & 00023 & 00018\end{array}$

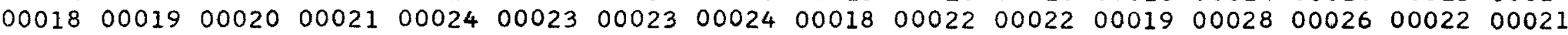
$\begin{array}{lllllllllllllllll}00026 & 00021 & 00037 & 00021 & 00021 & 00030 & 00017 & 00024 & 00029 & 00022 & 00019 & 00029 & 00035 & 00024 & 00034 & 00039\end{array}$

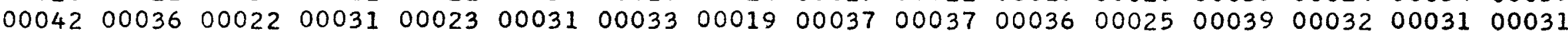
$\begin{array}{lllllllllllllllll}00037 & 00028 & 00038 & 00027 & 00040 & 00033 & 00040 & 00038 & 00047 & 00046 & 00044 & 00029 & 00050 & 00041 & 00071 & 00038\end{array}$ $0004100031000510004600040 \quad 00048 \quad 000470004200061000440004700052000430004700056 \quad 00057$

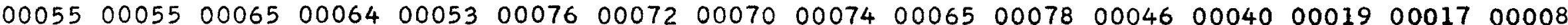
00014000060000200002000020000000000000000000000000000000000000000000070000006116

CRYSTAL HEIGHT $=06.000000$ IN. CRYSTAL RADIUS $=03.000000$ IN. $I=10000$ 00028000150001200023000160001900019000160002000017000140001400011000170002100028 $\begin{array}{llllllllllllllllll}00015 & 00020 & 00019 & 00013 & 00021 & 00020 & 00018 & 00021 & 00029 & 00017 & 00023 & 00014 & 00021 & 00016 & 00021 & 00015\end{array}$ $000190002100020 \quad 00025 \quad 00023 \quad 00022 \quad 00016 \quad 00018000210002200017 \quad 00024000250002600027 \quad 00024$ $\begin{array}{llllllllllllllll}00020 & 00022 & 00015 & 00026 & 00023 & 00023 & 00029 & 00030 & 00030 & 00029 & 00025 & 00023 & 00030 & 00029 & 00028 & 00034\end{array}$

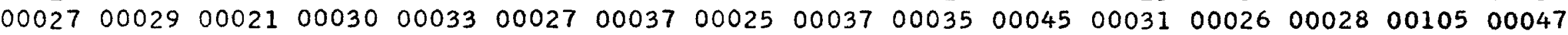
$\begin{array}{llllllllllllllllll}00040 & 00045 & 00050 & 00051 & 00045 & 00050 & 00039 & 00062 & 00063 & 00069 & 00066 & 00048 & 00052 & 00049 & 00061 & 00050\end{array}$ $000410004400058000690005800076 \quad 00061005050009500098000910008600096000860009800110$ 00104001180011000088000890003700036000180001200004000000000100000000020000704920

CRYSTAL HEIGHT $=06.000000$ IN. CRYSTAL RADIUS $=03.000000$ IN. $I=10000$ 000070001300015000050000900012000080001200009 $0002100009000150001200010 \quad 0001400011000180002100010000110002100011000110000900017$ $00017000190002000013 \quad 0001800016 \quad 000220001600010 \quad 00017000140001700015000150001300014$ $0000900010 \quad 00020 \quad 00020 \quad 000160001500023000240002200010 \quad 000160001200016000220001300015$ $\begin{array}{lllllllllllllllll}0 & 0 & 00022 & 00027 & 00031 & 00021 & 00020 & 00019 & 00027 & 00021 & 00021 & 00024 & 00024 & 00031 & 00021 & 00020 & 00029\end{array}$

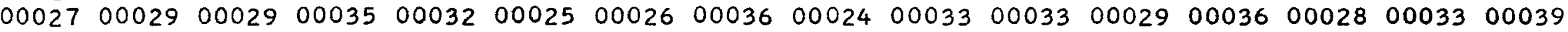

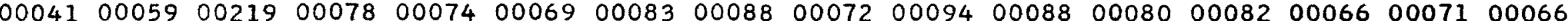

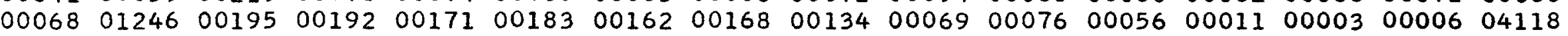

INCIDENT ENERGY $=04.450000 \mathrm{MEV}$. SOURCE HEIGHT $=00.000000$ IN. $A=04109 \quad P=01663$
0 $000120000900012000110001500008 \quad 000210001700019$ 
CRYSTAL HEIGHT $=06.000000$ IN. CRYSTAL RADIUS $=03.000000$ IN. $I=10000$

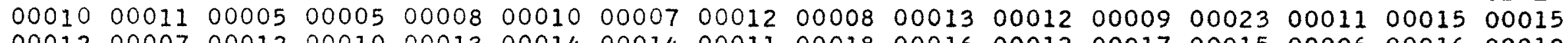
$00012000070001200010 \quad 00013000140001400011000180001600012000170001500006 \quad 00016 \quad 00010$ 00012000110001200009000100001200014000130000900014000060001700011000130001300012 $0001800010000080000900010 \quad 00008 \quad 00019000130001600008000110001700012000140001400019$ $000180001700016 \quad 000170001200006 \quad 00015000180001800013000130002500025000200002000023$ $0001700018000240002000027000220002400034000290002400028 \quad 00026 \quad 00023000180003400042$ $\begin{array}{llllllllllllllll}00022 & 00041 & 00030 & 00027 & 00029 & 00034 & 00039 & 00051 & 00046 & 00043 & 00262 & 00102 & 00105 & 00105 & 00110 & 00105\end{array}$ $00120000990007700089001100176500240 \quad 0025200264002600027200133000530001900002 \quad 03637$

CRYSTAL HEIGHT $=06.000000$ IN CRYSTAL RADIUS $=03.000000 \mathrm{IN}$. $I=10000$

000100001000006000050000600010000130001200010 $0000700008 \quad 0000700006 \quad 000120001400013000080001400009000090000800019000140001800013$ $0001600008 \quad 0001200015000190001700020000120001400017000190001400022000180001200019$ $000100001400010000120001500015000190001500020000130001300018000250001300016 \quad 00017$

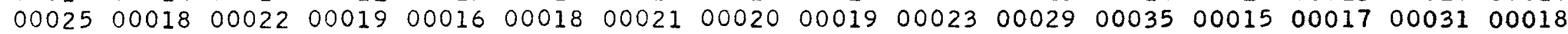

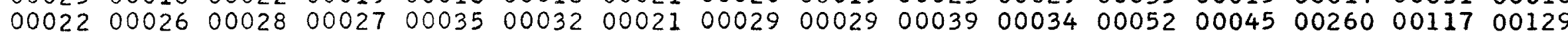

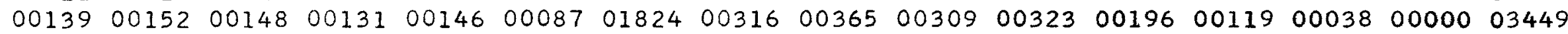

CRYSTAL HEIGHT $=06.000000$ IN. CRYSTAL RADIUS $=03.000000$ IN. $I=10000$

00007000060000700004000080000600005000110001100008000060000500010000090000500009 $\begin{array}{lllllllllllllllll}00011 & 00007 & 00009 & 00011 & 00012 & 00003 & 00010 & 00006 & 00015 & 00008 & 00013 & 00009 & 00010 & 00013 & 00012 & 00010\end{array}$ 00015000070001200008000090000600010000080001000006000090001100010000120001100012 $000130001300012000120001200010 \quad 00008 \quad 000130001600017000090001200014000100001200021$ 00012000250001300013000160001000021000110001400010000130001300015000180001300012 $00010 \quad 000090001900020 \quad 00025000230001900019000220001400020 \quad 00026 \quad 000240002600016 \quad 00037$ $0002300030 \quad 0002700029000290003400027000290002900031000440004700045000600006200296$ 00169001630017300174001680014100094019870040900366003250028300166000610000403281

INCIDENT ENERGY $=08.000000 \mathrm{MEV}$ SOURCE HEIGHT $=00.000000$ IN. $P=01525$

INCIDENT ENERGY $=07.100000 \mathrm{MEV}$. SOURCE HEIGHT $=0.0 .000000$ IN.

INCIDENT ENERGY $=06.130000$ MEV. SOURCE HEIGHT $=00.000000 \mathrm{IN}$. 7

(n)

.


TABLE III

COLLIMATED BEAM INCIDENT ON NaI CRYSTAL. BEAM RADIUS IS 1.693 IN.

CRYSTAL HEIGHT $=05.000000 \mathrm{IN}$. CRYSTAL RADIUS $=02.500000$ IN. $I=10000$

$0000100001000000000200004000000000100003000020000500003000030000400000 \quad 00003$ 00002000000000200000000010000100002000030000200001000040000000000000010000400002 00001000000000400003000030000300004000000000000000000020000100003000010000200005 00004000030000300009000060001200017000110001600006000170002100019000240002400020 $0002600027000330000300001000000000100000000000000200000 \quad 0000000000 \quad 0000200000 \quad 00000$ $0000100000 \quad 00000 \quad 00000 \quad 00000 \quad 0000000000 \quad 0000000000000000000000000000000000000000 \quad 00000$ $00000 \quad 00000 \quad 00000 \quad 0000000000 \quad 0000000000 \quad 000000000000000000000000000000000000000000000$ 00000000000000000000000280000200000000000000000000000000000000000000000000009544

CRYSTAL HEIGHT $=05.000000$ IN. CRYSTAL RADIUS $=02.500000$ IN. $I=10000$

$A=08098$

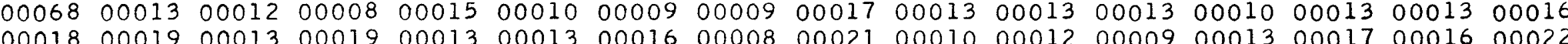
00018000160002000013000120001200018000130002500020000210001700013000160001900023 00018000240001900019000220002200025000170002000017000200001800027000130002000018 00018000230001100020000190002100023000230001800022000190002800023000240002400027 $0002400018 \quad 00020 \quad 00031 \quad 0002700030 \quad 00035000420003900039000460004900027000030000300005$ 00007000000000300005000040000100001000020000000000000000000000000000000000000000 $00000000000000000000 \quad 000000000000000000000000000000000000001200000000000000008098$

CRYSTAL HEIGHT $=05.000000$ IN. CRYSTAL RADIUS $=02.500000$ IN. $I=10000$

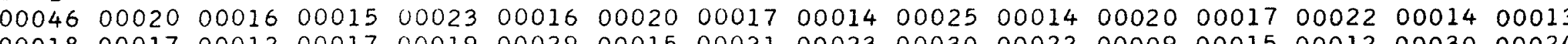
00018000170001200017000190002900015000210002300030000220000900015000120003000024 $000250002300020 \quad 000190002700029000150002100018000210002300036 \quad 000300002900034 \quad 00033$ $00030 \quad 0002300026 \quad 00026000190003100034000280002800033000340002900028000330004100025$ $00028000320003900031 \quad 00023 \quad 000260003700029000450004200030 \quad 0003500038 \quad 00046 \quad 00026 \quad 00039$ $0004300040 \quad 000350003700053000370003200036000220003300046 \quad 0003700044000450003500048$ $000450006400046 \quad 00048 \quad 0006200046 \quad 00056 \quad 000510005100022000230001600007000120000200004$ 00005000030000000000000000000100001000000000000000000000000000000000050000006765 SOURCE HEIGHT $=00.000000$ IN. INCIDENT ENERGY $=01.170000$ MEV
SOURCE HEIGHT $=00.000000$ IN. $P=00859$ INCIDENT ENERGY $=00.661000$ MEV. $P=00275$ 
TABLE III (CONTINUED)

CRYSTAL HEIGHT $=05.000000$ IN CRYSTAL RADIUS $=02.500000$ IN. $I=10000$

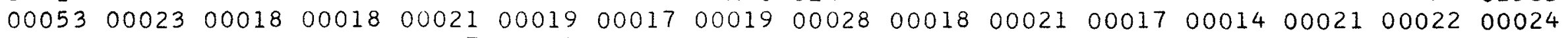
$\begin{array}{llllllllllllllll}00022 & 00018 & 00016 & 00023 & 00017 & 00027 & 00030 & 00017 & 00024 & 00022 & 00022 & 00018 & 00015 & 00023 & 00022 & 00023\end{array}$ $00016 \quad 0002500030 \quad 00032000150001900029000320001600028000310001600031000160002800029$ $0002300026 \quad 00020 \quad 0002400026 \quad 00020 \quad 000290002000061000290002700034000440003400026 \quad 00040$

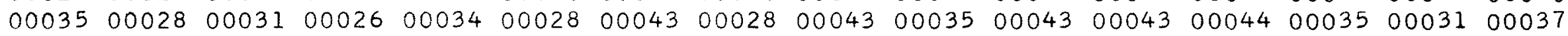
$000370004900041000380004400028000320004600053000460004600047002080006100053 \quad 00060$ $000700004900060 \quad 0006000050 \quad 0005800065000570006300056 \quad 0006300071000740006800070 \quad 00070$ 00052000210003300016000120000800001000020000300000000000000000000000000000305824

CRYSTAL HEIGHT $=05.000000$ IN. CRYSTAL RADIUS $=02.500000$ IN. $I=10000$

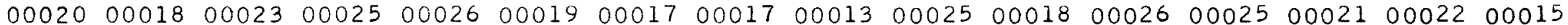
00016000200001200015000190002400019000190002500009000140002200023000190002800027 $0002100020 \quad 0001500022 \quad 00027 \quad 0002200019000210002400019000150001800033000300002000020$ 00019000250002100022000290002800022000160002400017000250001600019000230003000017

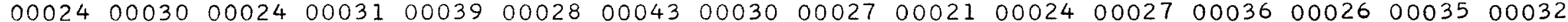
$00067000410004800040 \quad 000440006000054000390005300037000380004900056 \quad 000490006200046$ $000550005700040 \quad 000490006300051 \quad 000540005400549001040012400100 \quad 00104001150008500110$ 00096000920012600101000900005200036000240001100007000000000200001000000000405044

CRYSTAL HEIGHT $=05.000000$ IN. CRYSTAL RADIUS $=02.500000$ IN. $I=10000$

000120001300009000130001400009000130001400015 000200001300014000100001600012000090001600012 000160001300013000190001500014000210001200019

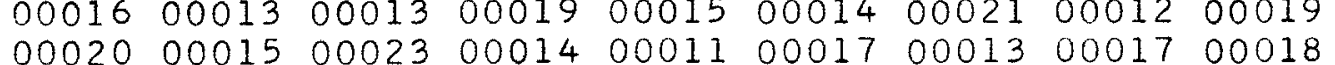
000160001800017000210001900015000 $00023 \quad 00026 \quad 0002100026 \quad 00024 \quad 00026 \quad 00027 \quad 0003300031$ $00026000450015500058 \quad 0007600076 \quad 000$ 000710112100182001670017700182
INCIDENT ENERGY $=04.450000$ MEV. SOURCE HEIGHT $=00.000000$ IN.

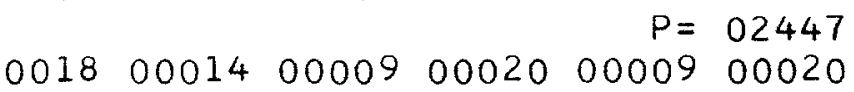
$\begin{array}{llllll}0013 & 00017 & 00021 & 00022 & 00019 & 00011 \\ 0026 & 00031 & 00020 & 00016 & 00014 & 00015\end{array}$ $\begin{array}{llllll}00021 & 00019 & 00025 & 00026 & 00018 & 00020 \\ 00020 & 00020 & 00019 & 00019 & 00016 & 00018\end{array}$ 000410002700021000420003400042 $\begin{array}{lllllllllll}00084 & 00079 & 00077 & 00089 & 00069 & 00067 & 00051 & 00069 & 00063 & 00067\end{array}$

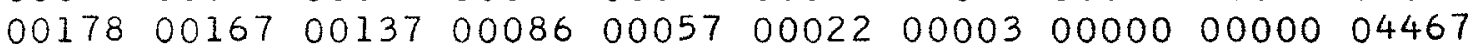


TABLE III (CONTINUED)

CRYSTAL HEIGHT $=05.000000$ IN. CRYSTAL RADIUS $=02.500000$ IN. $I=10000$ $000110000900011000150000600016 \quad 00014000090000700009000180001000005000130000900007$ $00013000090001100016 \quad 0001000015 \quad 00016 \quad 0001300020000150001300014000140001100016 \quad 00015$ 00015000090001500011000240002400020000110002100012000120001300010000100001100009 $000140001700014000090000900018 \quad 00020000170001900022000150002400014000190001900012$ $00020000190001500018 \quad 0001900026 \quad 00035000190001400029000260002200022000260002800034$ $\begin{array}{lllllllllllllllll}00024 & 00021 & 00029 & 00024 & 00029 & 00023 & 00036 & 00031 & 00038 & 00043 & 00203 & 00096 & 00107 & 00103 & 00109 & 00107\end{array}$ 00090000790009100082000730154100256002680026900290002540010000052000110000104091

CRYSTAL HEIGHT $=05.000000$ IN. CRYSTAL RADIUS $=02.500000$ IN. $I=10000$

00008000060000600006000080001300005000090001000004000100001300009000050000600014 $00009000140000700010 \quad 0000600001000120001100005000090001300006 \quad 000050001100006 \quad 00008$ 00005000090001100008000070000900009000090000900010000080000900009000130001200007 $0001300007000130000400010000040000900008 \quad 0001100020000100001300016000070000900009$ $000150001200008000160001500008 \quad 00009000100001600012000210001100016000140001300022$ $0001400016 \quad 000130000900016 \quad 0001400016 \quad 000140002000018000180001300021000210002300022$

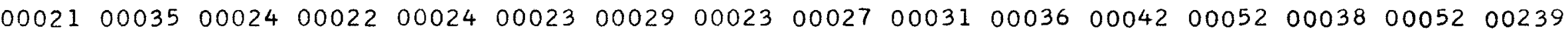
$0014400150 \quad 0017400135001330008100090 \quad 018100049300411003440030200154000600000403739$

CRYSTAL HEIGHT $=08.000000$ IN. CRYSTAL RADIUS $=04.500000$ IN. $I=10000$ $A=09653$

INCIDENT ENERGY $=00.279000$ MEV. SOURCE HEIGHT $=00.000000$ IN $00007000010000000000 \quad 000000000100000000020000000000000000000000002000010000100000$ 00002000000000000000000000000100000000000000100002000000000100000000010000100000 $0000100005000000000200000000010000200000 \quad 0000000000000000000100001000060000100003$ 00005000030000300005000060001300008000110001000008000140001600010000330001900025 $00035000130002100006 \quad 000000000300000000020000000002000010000100002000010000000000$ $0000000000 \quad 00000 \quad 00000 \quad 00000 \quad 00000 \quad 00000 \quad 00000 \quad 00000000000000000000000000000000000 \quad 00000$ $00000 \quad 00000 \quad 00000 \quad 00000 \quad 00000 \quad 00000 \quad 00000 \quad 00000 \quad 00000 \quad 0000000000 \quad 00000000000000000000 \quad 00000$ 00000000000000000000000220000100000000000000000000000000000000000000000000009653 
TABLE III (CONTINUED)

CRYSTAL HEIGHT $=08.000000 \mathrm{IN}$. CRYSTAL RADIUS $=04.500000$ IN. $I=10000$ 00015000020000400001000010000700004000070000000002000040000000001000020000300004 $0000200004000030000100000 \quad 0000000002000020000300003000010000100003000030000400001$ 00003000020000400003000020000400005000040000500003000050000200003000060000100004 00003000050000400002000060000100001000040000200001000050000200002000010000300008 00003000050000200006000040000100003000020000700009000110001800011000070000700018 $00021000280001800025000140001700030 \quad 000290005200034000530004600017000020000400006$ $000030000200002000010000100003000000000100000000000000000000000000000000000 \quad 00000$ 00000000000000000000000000000000000000000000000000000000000500000000000000009276

CRYSTAL HEIGHT $=08.000000$ IN. CRYSTAL RADIUS $=04.500000$ IN. $I=10000$

000190000500010000070000800005000050000300008 $000050000500004000050001300006 \quad 00007 \quad 00004000080008000100000900007000040000900005$ $0000900005000040000700006 \quad 00008 \quad 00005000040001200008000110001100003000050001000004$ $0000200015000090001400011000090001800006 \quad 0000900008000070000800013000080001100011$ 00014000120001400015000160001100010000090002200010000120000500004000090001600012 $00014000140001100007 \quad 00016 \quad 0001400011000090002000016 \quad 000150001500024000200002900024$ 00032000340002000035000410003700038000490005600026000160000700007000060000100002 $00003000020000000000 \quad 00000 \quad 00000 \quad 00000 \quad 000000000000000000000000000000000020000008651$

CRYSTAL HEIGHT $=08.000000$ IN. CRYSTAL RADIUS $=04.500000$ IN. $I=10000$

INCIDENT ENERGY $=01.830000$ MEV. SOURCE HEIGHT $=00.000000$ IN. $000210000900007000120001000007 \quad 00008000080000800010000120000500006000060000800009$ 00007000090000600009000160001400010000040001900010000130000900007000100000600009 00008000100000900007000060000700003000080001000005000140001300009000110000500009 00005000100001300009000170000900014000040000600014000090000800013000150001400015 $000140001900007 \quad 0001400010 \quad 0001200018000090000900017000170001500018000170001700020$ 00018000180002600010000190002000019000120001200020000190002300109000240002600032 $\begin{array}{lllllllllllllllll}00026 & 00021 & 00016 & 00026 & 00027 & 00030 & 00033 & 00031 & 00045 & 00027 & 00045 & 00044 & 00053 & 00041 & 00047 & 00054\end{array}$ 00045000170001900007000030000200000000010000000000000000000000000000000000308084 
TABLE III (CONTINUED)

CRYSTAL HEIGHT $=08.000000$ IN. CRYSTAL RADIUS $=04.500000$ IN. $I=10000$

000120001700006000050001000016000140001100012

00009000110000800011000100001700004000

00008000060001000012000050001100007000

00015000120001200007000140001800018004

00021000140001400019000270002300017000

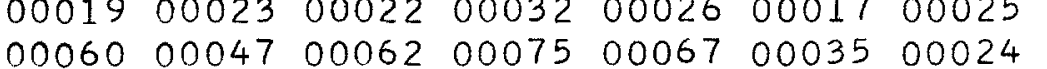

INCIDENT ENERGY $=02.760000$ MEV. SOURCE HEIGHT $=00.000000$ IN. $P=00645$ 00000016000050000800120001200015 (1) 000100001400008000070000900013000210001400014 $0001500010 \quad 00016 \quad 000140001100014000120001400014$ $000200002600016 \quad 00018 \quad 0001700014000240001800024$ $0003200303 \quad 00046 \quad 00037 \quad 0004900038 \quad 00050 \quad 0005900055$ 000090000900003000020000100001000000000107583
CRYSTAL HEIGHT $=08.000000$ IN. CRYSTAL RADIUS $=04.500000$ IN.

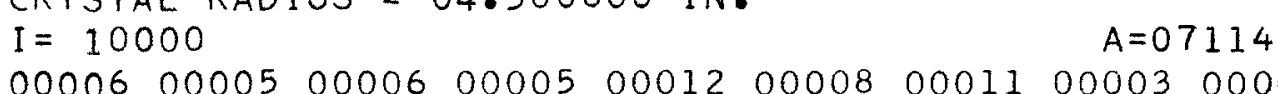
00008000110000700004000080000700007004 00005000100000600006000110000500009000090000800005 $00008 \quad 0000400008 \quad 0000900006 \quad 0000900011000090000900010$ 0001100012000170001000007000090000900012000100000900 00013000170001200009000190001500013000 00018000190003600025000310002300023000 00029006560012000110000970009800087
INCIDENT ENERGY $=04.450000 \mathrm{MEV}$. SOURCE HEIGHT $=00.000000$ IN. $P=00728$ 00030000800007000010001100008000090000600008 $0000600010 \quad 00005000060000700006 \quad 000090001400001$ 1000110000900010 000140000700010 000150000700009000120001100014 $\begin{array}{llllllllll} & 00019 & 00020 & 00015 & 00012 & 00019 & 00025 & 00013\end{array}$ 000370002900022000270003400028000280002400039 $0012000096 \quad 00044000320001000001000000000007117$
CRYSTAL HEIGHT $=08.000000$ IN. CRYSTAL RADIUS $=04.500000$ IN. $I=10000$

000080000500006000060000400005000030000800009 $00006000050000300007000090000400007 \quad 000040000600006 \quad 000070001000014000040000500007$ 00006000110000500004000040001000004000060000400008000100000400009000030000900012 $000050001100005000060000900006 \quad 00006 \quad 000050001400005000060000800007000090001000012$ 00010000100001000005000110000600006000160001100007000150001400010000140001100013 $\begin{array}{lllllllllllllllll}00016 & 00015 & 00021 & 00016 & 00014 & 00013 & 00027 & 00016 & 00026 & 00023 & 00038 & 00027 & 00038 & 00032 & 00044 & 00042\end{array}$ 00028000570003900046000340088600181001780016800184001580006800024000010000106849 $\begin{array}{ll}\text { INCIDENT ENERGY } & =06.130000 \text { MEV. } \\ \text { SOURCE HEIGHT } & =00.000000 \text { IN. }\end{array}$ $P=00811$ 
CRYSTAL HEIGHT $=08.000000$ IN. CRYSTAL RADIUS $=04.500000$ IN. $I=10000$

00004000010000200001000020000500003000030000500002000020000400007000030000600005 $0000800000 \quad 0000400005000040000700005000080000400007000050000700005000040000400002$

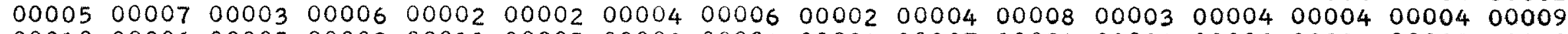
$00010 \quad 00006 \quad 0000500008 \quad 0001100005 \quad 00002 \quad 000040000400007 \quad 0000200003 \quad 00006 \quad 000110000200008$ $000050000300002000040000500006 \quad 00007000070000700007000100000800007000070001000008$ $00016 \quad 00004 \quad 00004 \quad 00007 \quad 0001100007 \quad 00006 \quad 00009000090001000008 \quad 0001000009000100000900015$ $00010000120001100010000120001700013 \quad 0001700016 \quad 000200001600017000140001900016 \quad 00044$ $00036 \quad 00045000440004400050 \quad 0004400038 \quad 0097100308002430022900202000950004500003 \quad 06790$

CRYSTAL HEIGHT $=12.000000$ IN. CRYSTAL RADIUS $=04.500000$ IN. $1=10000$ 000100000600003000020000300005000 000000000200001000000000200005 000060000300003000040000200006000 $\begin{array}{lllllllll}00005 & 00003 & 00012 & 00005 & 00007 & 00006 & 000 \\ 00010 & 00003 & 00005 & 00009 & 00016 & 00011 & 000\end{array}$ 000130001400032000290002000026000 000320061100101001170011600083
INCIDENT ENERGY $=04.450000$ MEV. SOURCE HEIGHT $=00.000000$ IN. $P=00198$ 000030000100002000010000300002000030000400005 000030000300003000050000400002000030000400003 $0000500005 \quad 00004 \quad 00004 \quad 0000400006 \quad 00005 \quad 0000100006$ 000060000100005000040000300004000070000500004 4000030000900009000090001300004000050001000008 $\begin{array}{llllllllll}0 & 0015 & 00012 & 00014 & 00007 & 00013 & 00008 & 00007 & 00018 & 00009\end{array}$ $\begin{array}{llllllllll} & 00024 & 00025 & 00020 & 00036 & 00031 & 00025 & 00032 & 00030 & 00024\end{array}$ $0011000099000470002000013000000000100000 \quad 07628$
CRYSTAL HEIGHT $=12.000000 \mathrm{IN}$. CRYSTAL RADIUS $=04.500000 \mathrm{IN}$.

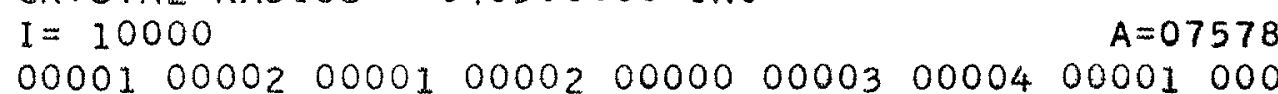
00002000020000100002000010003000040000100001 00003000050000200005000010000100003000030000400003 $000040000700000 \quad 00005000010000200005000050000200003$ 00003000030000400005000050000400003000040000200004 000030000800010000050000400001000080000800004 000120001300019000160001100009000110001200012 $00030 \quad 00027 \quad 00031000370003200798$
INCIDENT ENERGY $=06.130000 \mathrm{MEV}$. SOURCE HEIGHT $=00.000000$ IN. $P=00183$ $\begin{array}{lllllll}0002 & 00002 & 00004 & 00005 & 00002 & 00002 \\ 00004 & 00002 & 00002 & 00001 & 00002 & 00006\end{array}$ 000020000500001000050000400004 $0000500003 \quad 00002000020000400002$ 4000100001000006000050000700004 $\begin{array}{llllllllll}1 & 00002 & 00011 & 00012 & 00007 & 00007 & 00010\end{array}$ $\begin{array}{lllllll}00017 & 00030 & 00028 & 00023 & 00031 & 00026 & 00035\end{array}$ $\begin{array}{lllllllll}00136 & 00124 & 00051 & 00019 & 00003 & 00000 & 07580\end{array}$ 
TABLE III (CONTINUED)

CRYSTAL HEIGHT $=12.000000$ IN.

CRYSTAL RADIUS $=04.5$ COCCC IN. $I=10000$ $0000100001000020000300000 \quad 00002004$

00001000010000200000 c0000 0n002 00008

$000 \mathrm{c} 200000$ 0ccol 0000300004 00003

$000000000000005000 n 2000050600100002$

0000100004 crcos $0000300005 \quad n 0070000400$

$\begin{array}{llllllll}00009 & 00003 & 0 C 012 & 00007 & 00009 & 00012 & 00010 & 0 \\ 00027 & 00041 & 00046 & 00 r 39 & 00043 & 00039 & 00042 & 0\end{array}$
INCIDENT ENERGY $=8.000 C O C \mathrm{MEV}$.

SOLRCE HEIGHT $=0 C$. COCOCO IN.

$P=00191$

$0004000 C 300003 \quad 000020000500002000010000100003$

$000000000 \quad 0 C 0 C C \quad 00004 \quad 00005$ C00C6 C00CC 0000300001

$\mathrm{COC}^{2} 00003000010000000001 \mathrm{COOCl} 000040000200001$

000020000200001000010000100003000020000300002

CCOC4 $00006 \quad 000040000200002 \quad 00002 \quad 000020000300002$

000030000500006000040000500006000050000500009

$000 c 80001100011000110000900019000150001900041$

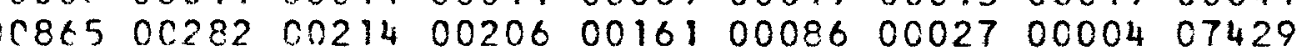


TABLE IV

COLLIMATED BEAM INCIDENT ON NaI CRYSTAL. BEAM RADIUS IS 0.250 IN.

CRYSTAL HEIGHT $=07.250000$ IN. CRYSTAL RADIUS $=02.500000 \mathrm{IN}$. $I=05000$

0003100004000050000300006000040000200 $000000000200005000060000400000 \quad 00003$ 00007000040000500004000060000500003 00006000100000900005000030000300002 00008000060000400008000110000300006000 $\begin{array}{lllllllll}00010 & 00008 & 00011 & 00009 & 00011 & 00012 & 00017 & 004 \\ 00002 & 00002 & 00001 & 00000 & 00001 & 00001 & 00002 & 001\end{array}$ 00000000000000000000000000000000000
$A=04385$

INCIDENT ENERGY $=00.661000 \mathrm{MEV}$. SOURCE HEIGHT $=00.000000$ IN. $P=00030$

000050000200000000000000300002000010000100005 $0000100003 \quad 000050000100002 \quad 00003 \quad 00007 \quad 0000400003$ 000030000500004000040000700004000040000700008 $0000500008 \quad 0000700013 \quad 0000400011000040000700004$ 000030000500011000010001100015000090000800008 $0001200016 \quad 00020000250001800006 \quad 00003 \quad 0000200002$ $00000 \quad 00000 \quad 00000 \quad 0000100000 \quad 00000 \quad 00000 \quad 00000 \quad 00000$ $0000000000 \quad 00000 \quad 000000000200000000000000004385$
CRYSTAL HEIGHT $=07.250000$ IN. CRYSTAL RADIUS $=02.500000 \mathrm{IN}$. $I=05600$

000260000900008000070000500004000050000700006 000060000800004000030000700003000120000900003 00007000070000800006000020000500007 00009000090001100007000160000900016000 00015000180001300012000150001700017004 0001200015000100001500007000230001500 $\begin{array}{lllllllll}00028 & 00021 & 00017 & 00021 & 00028 & 00023 & 00027 & 004 \\ 00000 & 00000 & 00000 & 00000 & 00000 & 00000 & 00000 & 000\end{array}$
INCIDENT ENERGY $=01.119000 \mathrm{MEV}$. SOURCE HEIGHT $=00.000000$ IN. $P=00137$ $0000700006 \quad 00006 \quad 000100000400003 \quad 000050000200003$ $00005000050000400011000080000400010 \quad 0001200007$ 000080001300011000100000800008000100001100018 $0001600017 \quad 00016 \quad 000130001500014000150001900012$ $0001200016 \quad 00018000150002000020000310001700020$ $0003700016 \quad 000110000200006 \quad 00003 \quad 00002 \quad 0000500001$ 000000000000000000000000000000000020000004350
CRYSTAL HEIGHT $=07.250000$ IN. CRYSTAL RADIUS $=02.500000$ IN. $I=06400$

$A=04346$

INCIDENT ENERGY $=01.780000 \mathrm{MEV}$. SOURCE HEIGHT $=00.000000$ IN. 00020000030000500007000030000700005000040000400005000010001300011000060000700004 $000090000600008 \quad 0000900005000090001200006 \quad 0000600008000050000600003000070001100011$ 00014000060001000012000050000500007000070000700019000090001300009000140001200010 $00006 \quad 000120001200007 \quad 000140001500016000120001600015000090001100013000170001500012$ 00017000200001800018000170001500021000200002200020000180001300022000270002100026

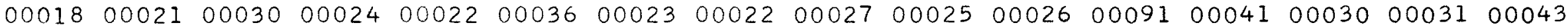
$0002500034000450003100034000330003900033000310004100037000390004300046 \quad 00036 \quad 00042$ $000140001700005000120000200000 \quad 00000000000000000000000000000000000000000000304346$ 
TABLE IV (CONTINUED)

CRYSTAL HEIGHT $=07.250000$ IN. CRYSTAL RADIUS $=02.500000 \mathrm{IN}$. $I=07000$ 00012000070000400009000050000500006000080001000014000070000800009000120000100007 00006000090000800007000080000700006000020000600006000040000600008000110001100012 $0001000008 \quad 0001300009000070000800004000120001000006000040001000009000080001100010$ 00011000150000900016000120001400014000090001000007000090000900011000120001700008 00016000090001500010000210001300021000160001600019000170002000011000160001700018

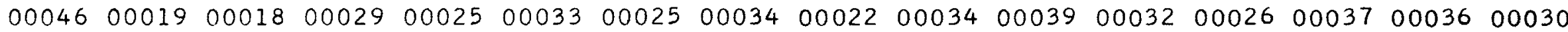
$00031000320002700040 \quad 00042000330004100048 \quad 0033200070000710004900062000640005900079$ 00079000540007400062000610003800019000150000800004000000000000000000000000204201

CRYSTAL HEIGHT $=07.250000$ IN. CRYSTAL RADIUS $=02.500000 \mathrm{IN}$.

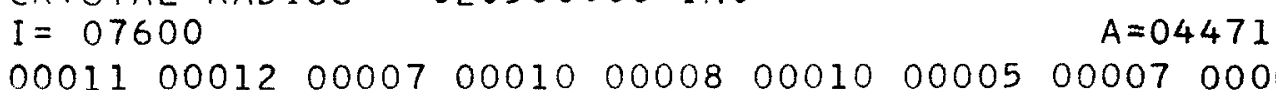

0000000010000050000700007

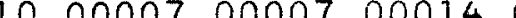

00006000070000600007000070000700015

0001400011000150001400010000140001500

$000160001600018 \quad 0002500024 \quad 004$

0003800037000390003700026000

0007400068000630006800098
CRYSTAL HEIGHT $=07.250000$ IN. CRYSTAL RADIUS $=02.500000 \mathrm{IN}$. $I=08050$

000120000800012000060000600008000070000500010 00009000180000300005000040000900008000060000800006000100000900010000080001100009 00008000060000800006000030001100007000110001600010000120000700012000090001200009 00014000120000700007000090001200014000090001200010000170000900008000110001400011 00012000090002400016000080001200013000170000900020000160001600010000180001900019 00026000240002400019000270001700026000210002700047000320003500030000340003900050 $0003700038000390003500050 \quad 0003900041000450004500050 \quad 000470004200490 \quad 0008000076 \quad 00104$ 00106001030008000089000980008400090000390002500013000080000300000000000000004751

INCIDENT ENERGY $=03.425000$ MEV. SOURCE HEIGHT $=00.000000$ IN. 000500010000060001000011000100000800008000 $A=04201$

CIDENT ENERGY $=02.753000 \mathrm{MEV}$ SOURCE HEIGHT $=00.000000$ IN.
INCIDENT ENERGY $=03.065000 \mathrm{MEV}$. SOURCE HEIGHT $=00.000000$ IN. $\begin{array}{rr}P= & 00707 \\ 0010 & 00009\end{array}$ 60001500007000120001000009 $\begin{array}{lllllll}5 & 00005 & 00008 & 00007 & 00012 & 00011\end{array}$ $\begin{array}{llllll}0 & 00010 & 00007 & 00013 & 00012 & 00011\end{array}$ 10001200015000120001400010 $\begin{array}{lllllll}7 & 00024 & 00018 & 00017 & 00026 & 00021\end{array}$ $\begin{array}{lllllll}2 & 00038 & 00035 & 00047 & 00033 & 00040\end{array}$ 0009100080000850008000096 00000100002000000000504471

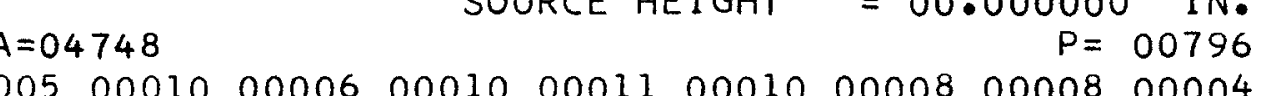


TABLE IV (CONTINUED)

CRYSTAL HEIGHT $=07.250000$ IN. CRYSTAL RADIUS $=02.500000 \mathrm{IN}$. $I=10000$

$00009000080001500008000050001200010 \quad A=05711$ $0001100010 \quad 00012000120001300006 \quad 00011000700018$ 00010000050001100018000100001500013000150001600 00008000110001000012000170001700012 000110001700013000170001500017000130001400016 $00022000150002800026 \quad 00017 \quad 00033 \quad 00024000280002800$ $\begin{array}{llllllllll}00040 & 00048 & 00065 & 00051 & 00042 & 00052 & 00070 & 00061 & 00056 & 00 \\ 00136 & 00144 & 00143 & 00134 & 00131 & 00121 & 00127 & 00110 & 00061 & 000\end{array}$
INCIDENT ENERGY $=03.805000 \mathrm{MEV}$. SOURCE HEIGHT $=00.000000$ IN. $\begin{array}{rllll}P & =00962 \\ 00008 & 00010 & 00005 & 0000500010 & 00007\end{array}$ $\begin{array}{llllllll}0008 & 00008 & 00010 & 00005 & 00005 & 00010 & 00007 \\ 0010 & 00010 & 00008 & 00012 & 00007 & 00014 & 00011\end{array}$ 00014000130001300011000090001100005 00013000130001200011000100001400016 00020000130001800031000210001600022 $0003600030 \quad 00024000300008300056 \quad 00054$ 00054000630005800053000510072000126 00035000150000700003000000000005714
CRYSTAL HEIGHT $=07.250000$ IN. CRYSTAL RADIUS $=02.500000 \mathrm{IN}$. $I=10000$

000070001000006000150000900006000080000900007 000050000800009000090000900006000110001000005 $0000900006000120000700006 \quad 00006 \quad 000170000800008$ 000140000800005000110000900012000130000600018 000140001100012000150001600011 $00016000140003100024000210003300028 \quad 0002600024$ $\begin{array}{lllllllll}00083 & 00058 & 00051 & 00064 & 00073 & 00057 & 00055 & 00072 & 00067 \\ 00884 & 00207 & 00154 & 00144 & 00150 & 00170 & 00129 & 00135 & 00093\end{array}$
INCIDENT ENERGY $=04.205000 \mathrm{MEV}$. SOURCE HEIGHT $=00.000000$ IN. $P=00985$ 00013000080000400004000090001100010 00008000110001000006000110000500008 00011000150001100011000080001100013 $\begin{array}{llllllll}00015 & 00013 & 00008 & 00011 & 00013 & 00020 & 00014\end{array}$ 00015000200001000018000230001900019 $000290001700013000240003500036 \quad 00035$ 00061000520005700072000610006700058 00060000240001900002000000000005540
CRYSTAL HEIGHT $=07.250000$ IN. CRYSTAL RADIUS $=02.500000 \mathrm{IN}$. $I=10000$

$A=05469$

00007000040000900010000060001400011000100001100008000100001200009000120001000015 00014000080000800011000140000700007000090001200010000080001100007000160000700012 00006000090000900007000080000300008000100001400011000110000900006000100001200010 $00009000070001000010 \quad 00009000110001500008 \quad 0001600017000080000800014000170002600016$ 00011000160001000011000150001900014000150001200014000130001500026000130001300024

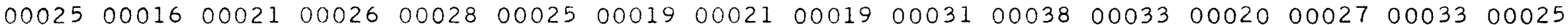
$00030 \quad 000240002900078 \quad 0006300048 \quad 00046 \quad 000520006100065000810006300069000640006200063$ 00052009330021100162001760015600158001900012400091000430001700007000010000005470

INCIDENT ENERGY $=04.625000$ MEV. SOURCE HEIGHT $=00.000000 \mathrm{IN}$. $P=01040$ 
TABLE IV (CONTINUED)

CRYSTAL HEIGHT $=07.250000$ IN. CRYSTAL RADIUS $=02.500000$ IN $I=09350$

$0000800008 \quad 0001100006 \quad 000090000800007 \quad 000090001200005000050000800009000090000900009$ 00004000040000700012000090000800006000060000200008000080000500005000160001100014 00010000080001400004000150000600011000100001200007000090001500007000120000800013 $0001200012000120001600011000080001900008 \quad 00017000060001800010000150002200008 \quad 00015$ 00011000130001800020000190001500013000240001800019000230003600021000190001700026 $00030 \quad 00016 \quad 0002900027 \quad 000350003300125000610006400042000750006500070 \quad 0006400068 \quad 00065$ 00045000580006001080002020017800170001830016000121000770002300010000030000005058

CRYSTAL HEIGHT $=07.250000$ IN. CRYSTAL RADIUS $=02.500000 \mathrm{IN}$. $I=09600$

$A=05162$

00009000090000600011000110000200011000070000900005000040000700004000050000500008 00006000150000900008000030000800013000060000900006000040000500005000040000400009 00006000020000800007000110001000010000050000900017000050000300011000040001200009 $0000700006 \quad 00015000080000900007 \quad 00005000060000500006000070000900008000110001000014$ 00008000110001100016000100001500012000100001400009000200001200008000180001300018 $0001400014000110001600020 \quad 0001600012000180002300026000130002000019000240001500024$ $0001700021000250002800030 \quad 00038 \quad 0002900034001230007400057 \quad 00065000670008000087 \quad 00064$ 00063000630007500069011440024900217001700017900202001140004900013000010000105173

CRYSTAL HEIGHT $=07.250000$ IN. CRYSTAL RADIUS $=02.500000$ IN. $I=09800$

\section{$A=05170$}

INCIDENT ENERGY $=06.005000 \mathrm{MEV}$. SOURCE HEIGHT $=00.000000$ IN.

00005000050000600006000040000600005000040000500003000060000500009000050000100002 00007000110000800009000050000500004000060000600011000070000600006000050001000006 $0001000009000040000300009000080000600008000110000400006 \quad 0000400010000040000100008$ $00014000080000900006 \quad 00006 \quad 0000500006 \quad 000070000700002000090000700012000040000600007$ 00008000030000700010000080001200009000150001200012000110001800013000060001300010 00012000250001000008000150001600013000180001800021000170002000017000230001700026 $00021000220003200030 \quad 00035000340002000034000340003700128000700007300078 \quad 0009100107$ $00070 \quad 000730004700057000660136200247002560021900249001720007500033000020000005174$ 
TABLE IV (CONTINUED)

CRYSTAL HEIGHT $=07.250000$ IN. CRYSTAL RADIUS $=02.500000$ IN.

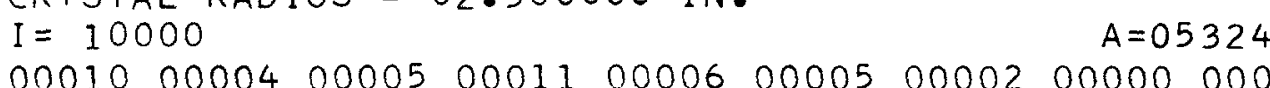

$00008000020001000006 \quad 0000200007 \quad 000130000600007$

000040001000009000060000500006000130000500008006

000050001000008000090001600008000

$\begin{array}{llllllll}0 & 0005 & 00007 & 00009 & 00010 & 00006 & 00014 & 00010 \\ 00008 & 00006 & 00013 & 00018 & 00011 & 00017 & 00016\end{array}$

$\begin{array}{lllllll}00029 & 00022 & 00028 & 00019 & 00020 & 00024 & 000 \\ 00081 & 00063 & 00068 & 00064 & 00077 & 01184 & 003\end{array}$
INCIDENT ENERGY $=06.505000 \mathrm{MEV}$. SOURCE HEIGHT $=00.000000 \mathrm{IN}$. $P=01084$ $\begin{array}{llllllll}0006 & 00011 & 00006 & 00008 & 00010 & 00005 & 00008 \\ 0006 & 00005 & 00008 & 00009 & 00007 & 00005 & 00005\end{array}$ $\begin{array}{llllllll}0006 & 00005 & 00008 & 00009 & 00007 & 00005 & 00005 \\ 0003 & 00005 & 00012 & 00008 & 00008 & 00006 & 00006\end{array}$ 00008000110001100005000050000800006 00013000110000600007000080001300006 00009000180001900021000190002000022 00035000350011600086000760009400081 $002520022200149000450001200000 \quad 05332$
CRYSTAL HEIGHT $=07.250000$ IN. CRYSTAL RADIUS $=02.500000 \mathrm{IN}$. $I=10000$

$A=05230$

000030000700006000060000700004000030000400007

00003000100000100005000050000100

000090001300006000040000700008004

$00008 \quad 0000900011000130000800013$

$000150001000018000130001300010 \quad 00$

$\begin{array}{llllll}00021 & 00018 & 00028 & 00025 & 00022 & 00020 \\ 00095 & 00087 & 00072 & 00063 & 00092 & 00082\end{array}$
INCIDENT ENERGY $=07.025000 \mathrm{MEV}$. SOURCE HEIGHT $=00.000000 \mathrm{IN}$. $P=01046$ 700004000090000800003000080000800010 0000700004000090000800003000080000800006 $0001000006 \quad 00008 \quad 000020000500008 \quad 0000900007 \quad 00006$ $00006 \quad 00008 \quad 0001000008 \quad 00010000120001800008 \quad 0000500013$ $0001700016 \quad 0001400018000170002200021000120002100024$ 00025000350002300032000410003700030001430010700094 01270003030035500304002640018200069000200000005233
CRYSTAL HEIGHT $=07.250000$ IN. CRYSTAL RADIUS $=02.500000$ IN. $I=10000$

000080000200006000060000600007000050000400006 00008000060000400006000070000800 000050000300005000060000600004000 000100001000007000120000900005000 000060000900005000070001100006000 $\begin{array}{llllllll}00005 & 00010 & 00011 & 00008 & 00014 & 00018 & 008 \\ 00021 & 00027 & 00019 & 00027 & 00021 & 00027 & 008\end{array}$ 000970012200111000990010600083
INCIDENT ENERGY $=07.565000 \mathrm{MEV}$. SOURCE HEIGHT $=00.000000$ IN.

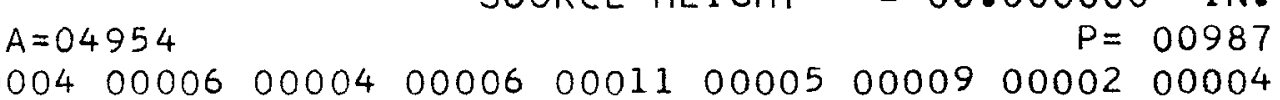
$0000200006 \quad 00007000050000900010000050000400009$ 000120000500004000050000700003000080000700008 $0000700006 \quad 00009000070000600004000040000700008$ 000090000900011000120001000010000100000900016 $\begin{array}{lllllllllll}00016 & 00013 & 00014 & 00018 & 00019 & 00015 & 00008 & 00021 & 00016\end{array}$

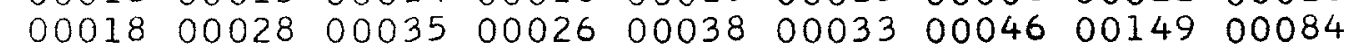
$\begin{array}{llllllllll}01580 & 00397 & 00339 & 00277 & 00232 & 00098 & 00031 & 00002 & 04955\end{array}$ 
TABLE IV (CONTINUED)

CRYSTAL HEIGHT $=07.250000$ IN. CRYSTAL RADIUS $=02.500000$ IN. $I=10000$

00003000040000400003000050000300004000030000300004000050001000008000020000800009 00005000010000600003000030000300004000090000600007000040000100004000060000400005 00006000090000900003000080000600007000060000600002000050000500005000050000800003 $000060001100006 \quad 0000700009000090000700008 \quad 000040000500008 \quad 0000800004000060000200007$ 00006000080000900012000080001300009000120001100006000080000900004000060001200005 $000100000800004000190001400016 \quad 00013000110001300011000140001400011000200001200019$ 00016000180001500019000200002600026000160003000041000320002400037000460003900114 $0008500113001010011100100 \quad 00094000870137500465004170037400278001530004100003 \quad 04987$

CRYSTAL HEIGHT $=07.250000$ IN. CRYSTAL RADIUS $=02.500000$ IN. $I=10000$ 00080000700003000020001000001000030000500002 00005000020000400004000150000500007000040000900004000040000200005000030000400003 00006000040000700003000020000300008000050000400002000030000100005000060000200009 00006000050000200007000070000800003000070000100008000040000800004000070000700010 00008000110000500010000050001200005000080000500011000030000600011000050000200009 $0000500011000070000400006 \quad 0000500008 \quad 000090001400007000180001400016000070000900008$

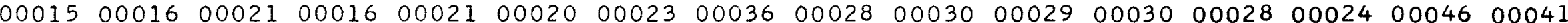
$0015100116001330012900100001 J 100118001260161400463004030035400184000460000204942$

CRYSTAL HEIGHT $=07.250000$ IN. CRYSTAL RADIUS $=02.500000 \mathrm{IN}$. $I=10000$

00007000080000100006000040000100001000020000400003000080000800005000080000600004 00003000050001000005000040000200006000090000300007000070000300004000050000200004 $000020000300000 \quad 00001000030000600005000140000400003000050001000003000110000400005$ $0000700005000020000400003 \quad 00008 \quad 00006 \quad 000040000400004000080000300008000030001000002$ 00003000090000200005000060000300006000050001000007000060000600006000090000500005 00009000080000700007000070000300013000050001000012000100001000012000160001000010 $\begin{array}{llllllllllllllll}00020 & 00014 & 00024 & 00020 & 00025 & 00021 & 00028 & 00022 & 00024 & 00019 & 00025 & 00023 & 00028 & 00031 & 00031 & 00047\end{array}$ $00040 \quad 001450013200144001330010900143001430146400517005210037300265000850000404823$
INCIDENT ENERGY $=09.305000 \mathrm{MEV}$. SOURCE HEIGHT $=00.000000$ IN. $P=00928$

INCIDENT ENERGY $=08.705000 \mathrm{MEV}$ SOURCE HEIGHT $=00.000000 \mathrm{IN}$. $P=00968$ 
TABLE IV (CONTINUED)

CRYSTAL HEIGHT $=07.250000$ IN. CRYSTAL RADIUS $=02.500000$ IN. $I=10000$

$000060000400001000030000500006 \quad 00000000020000600004000060000500002000050000500005$ 00008000020000500003000050000100003000050000500003000060000100003000060000300006 $00005000020000200005000040000500003 \quad 0000200004000030000300007000040000700006 \quad 00007$ 00005000080000300007000100001100003000040000700006000060000400008000050000900004 00009000100000700007000060000500005000040001000005000050000600008000090001300008 00012000030000400009000090000800017000120000700010000170001100007000110000900013 00024000120001400017000120002000014000230001900016000280002600016000370003400038 00055000590013000151001300013400189001540013001758005130042500345001170001004775

CRYSTAL HEIGHT $=07.250000$ IN CRYSTAL RADIUS $=02.500000$ IN. $I=10000$ 070000300003000020000200002010050000400005000010000200005000060000300006 $00007 \quad 00002 \quad 00007 \quad 000040000400002 \quad 00001000080000400005000050000300006000040000400004$ $00008000040000500003000020000500000 \quad 000050000700006000080000300007000020000400007$ 00008000030000600004000030000600007000060000300007000040000700005000070000800011 $00009000040000400004000090000700006 \quad 000070000900013000160001700007000040001800010$ 00011000170001400016000190001700023000150003000036000280002800023000220002900049

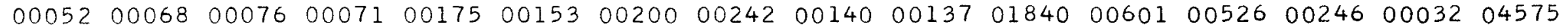

INCIDENT ENERGY $=11.700000$ MEV. SOURCE HEIGHT $=00.000000$ IN. $P=00844$

\section{$A=04571$}


TABLE $V$

DISC SOURCE ON CRYSTAL AXIS OF NAI CRYSTAL

CRYSTAL HEIGHT $=03.000000$ IN.

CRYSTAL RADIUS $=01.500000$ IN.

$T=37409$

$I=10000$

$0017400038 \quad 000410004500051 \quad 00048 \quad 0003900036 \quad 000490004500040 \quad 0003300047000410002500046$

$000310004500048 \quad 00041 \quad 00046 \quad 0004300042 \quad 00030 \quad 0004800033000330003900033000440004100054$

$00040 \quad 0005400046 \quad 0003900049000340005500041000420003900043 \quad 0004400039000410004400030$

$00045000340003900036 \quad 0004200043000390004400030 \quad 00036 \quad 00046 \quad 0004700045 \quad 0005200035 \quad 00042$

$00038 \quad 000410003900047 \quad 0003500053 \quad 00048 \quad 0004100026 \quad 000380004900042000510006500046 \quad 00043$

$000570005200051000470004800053 \quad 000580006100061000600006500062000350002300016 \quad 00015$

$00008 \quad 000060001200003000020000500003000020000200002000010000000000000000000000000$

00000000000000000000000000000000000000000000000000000000002800000000000000005676

CRYSTAL HEIGHT $=03.000000$ IN CRYSTAL RADIUS $=01.500000$ IN. $T=45131$

$I=10000$

$A=03889$

INCIDENT ENERGY $=01.330000 \mathrm{MEV}$. SOURCE HEIGHT $=03.936992$ IN. $00114000380004400040 \quad 000450004900045000470004600038 \quad 0003200044000460002500038 \quad 00040$ $0005100046 \quad 00040 \quad 000420004300036 \quad 0003900032000440003900043 \quad 000420004500056 \quad 00036 \quad 00041$ $000570004200040 \quad 0003400052 \quad 0004700050 \quad 00038000480004900046 \quad 00046 \quad 00043000420003300040$ $\begin{array}{llllllllllllllllll}0 & 053 & 00060 & 00038 & 00054 & 00053 & 00037 & 00049 & 00035 & 00046 & 00049 & 00047 & 00058 & 00057 & 00049 & 00056 & 00061\end{array}$ $00040 \quad 00058 \quad 00050 \quad 00051 \quad 0003900052 \quad 0004700046 \quad 00058 \quad 0006300066 \quad 000470005500058 \quad 0007200068$ $\begin{array}{lllllllllllllllll}00060 & 00057 & 00080 & 00057 & 00059 & 00073 & 00074 & 00077 & 00068 & 00079 & 00056 & 00081 & 00081 & 00066 & 00066 & 00077\end{array}$ $0008600085000850007700079001170009900093001060008900106 \quad 0005700036000310001400015$ 00007000080000300007000020000100000000000000000000000000000000000000110000103889

CRYSTAL HEIGHT $=03.000000$ IN. CRYSTAL RADIUS $=01.500000$ IN. $T=53127$ $I=10000$

INCIDENT ENERGY $=02.620000 \mathrm{MEV}$. SOURCE HEIGHT = 03.936992 IN.

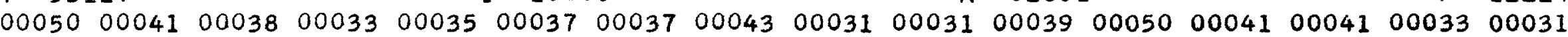

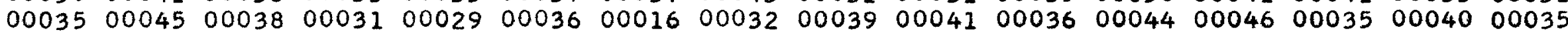

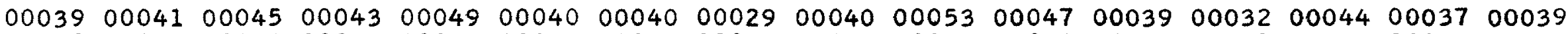
$00040 \quad 00048 \quad 00030 \quad 00043 \quad 0003900047 \quad 00051 \quad 00045000450004400040 \quad 00045000470004300038 \quad 00039$

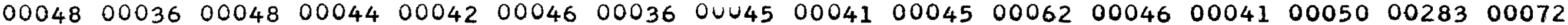

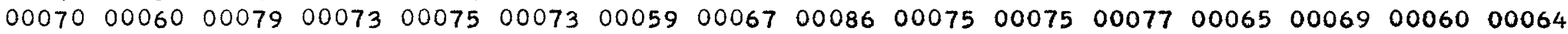
$\begin{array}{lllllllllllllllll}0 & 00064 & 00059 & 00073 & 00090 & 00088 & 00088 & 00559 & 00105 & 00113 & 00099 & 00111 & 00123 & 00100 & 00123 & 00117\end{array}$ 00118001330013400138001110004200029000240001100009000000000700003000040000902602 
TABLE V (CONTINUED)

CRYSTAL HEIGHT $=03.000000$ IN. CRYSTAL RADIUS $=01.500000$ IN. $T=57249$

$I=10000$

000320002700033000290002900037000

0001800031000320003200033000290003

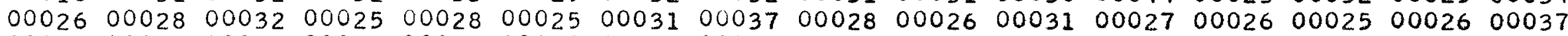
$\begin{array}{lllllllllllllllllllll}00029 & 00032 & 00038 & 00033 & 00033 & 00030 & 00027 & 00031 & 00033 & 00026 & 00032 & 00025 & 00023 & 00043 & 00034 & 00033\end{array}$ $0002400030 \quad 00024 \quad 00041 \quad 00032000280004100031000360004500046 \quad 0003500040 \quad 000400004400043$

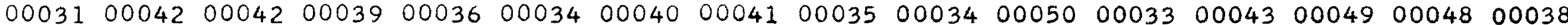
$000430006100572 \quad 001340013400129001150012900135001230011900113001010007900099 \quad 00094$

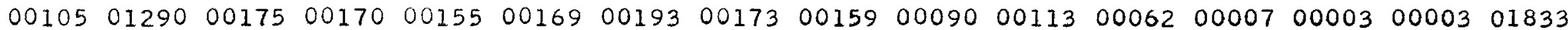

CRYSTAL HEIGHT $=03.000000$ IN. CRYSTAL RADIUS $=0 I .500000$ IN. $T=56505$ $I=10000$ $A=01397$

INCIDENT ENERGY $=06.130000 \mathrm{MEV}$. $0002200025 \cdot 00023000280002000012$ 000310001500019000220002400033 $00028000290001900030 \quad 000220002700030$ $00026 \quad 00030 \quad 0002900029000260003900020$ $00028 \quad 00023 \quad 0002400035000340002900033$ $00038 \quad 00040 \quad 00042000340003400030 \quad 00029$ $000470004400048 \quad 0004200048 \quad 0003500052$ $\begin{array}{llllllll}00173 & 00166 & 00089 & 00141 & 00127 & 01670 & 00231\end{array}$ $\begin{array}{ll}\text { INCIDENT ENERGY } & =04.450000 \mathrm{MEV} \bullet \\ \text { SOURCE HEIGHT } & =03.936992 \mathrm{IN} \text {. }\end{array}$ $\begin{array}{rl}A=01823 & P=13985\end{array}$ 
TABLE V (CONTINUED)

CRYSTAL HEIGHT $=03.000000$ IN. CRYSTAL RADIUS $=01.500000 \mathrm{IN}$. $T=55994$ $I=10000$

$00007000120001600007 \quad 000220001500$ $00016 \quad 000240002000015000220002300023000180002300017$ $00028 \quad 0001800017000250001500020000260001800030$ $000250002300032 \quad 000220002500028000200002200034$ $\begin{array}{llllllllll}00033 & 00026 & 00030 & 00026 & 00022 & 00025 & 00039 & 00036 & 00032 & 00 \\ 00018 & 00029 & 00027 & 00037 & 00026 & 00029 & 00033 & 00030 & 00035 & 00\end{array}$ 0003300035000310004100034000540004 00110008860037100336002370024700355
INCIDENT ENERGY $=09.000000 \mathrm{MEV}$ $A=00986$ SOURCE HEIGHT $=03.936992$ IN. $P=13286$ 022000170002500018000190002000019 $\begin{array}{lllllllll} & 00016 & 00021 & 00032 & 00018 & 00022 & 00013\end{array}$ $\begin{array}{llllllllll}00031 & 00020 & 00026 & 00022 & 00020 & 00016 & 00027\end{array}$ 00029000350003500020000350002900025 $\begin{array}{llllllll}00034 & 00026 & 00017 & 00030 & 00017 & 00036 & 00025\end{array}$ $\begin{array}{lllllllll}0.0034 & 00035 & 00032 & 00033 & 00035 & 00040 & 00034\end{array}$ $\begin{array}{lllllllllll}0 & 00047 & 00058 & 00056 & 00071 & 00055 & 00089 & 00081 & 00152 & 00156\end{array}$ $\begin{array}{lllllllll}00158 & 01884 & 00284 & 00288 & 00199 & 00156 & 00091 & 00008 & 00993\end{array}$
CRYSTAL HEIGHT $=03.000000$ IN. CRYSTAL RADIUS $=01.500000 \mathrm{IN}$. $T=54830$ $I=10000$ $A=00757$

INCIDENT ENERGY $=11.000000 \mathrm{MEV}$ SOURCE HEIGHT $=03.936992$ IN. 00012000120001000008000140001100017000130002300008000260001300016000150001800020

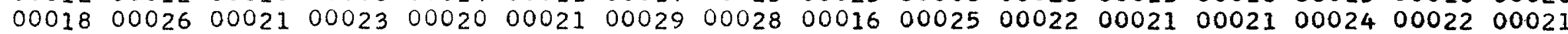

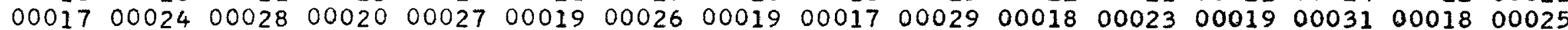
$\begin{array}{llllllllllllllll}00020 & 00020 & 00020 & 00027 & 00027 & 00021 & 00028 & 00029 & 00044 & 00028 & 00033 & 00026 & 00033 & 00025 & 00036 & 00032\end{array}$ $\begin{array}{llllllllllllllll}00031 & 00030 & 00025 & 00029 & 00031 & 00033 & 00040 & 00033 & 00030 & 00027 & 00035 & 00032 & 00019 & 00032 & 00039 & 00031\end{array}$ $\begin{array}{llllllllllllllll}00033 & 00040 & 00028 & 00023 & 00033 & 00035 & 00028 & 00025 & 00032 & 00027 & 00032 & 00039 & 00029 & 00032 & 00030 & 00033\end{array}$ $00036 \quad 00036 \quad 00040 \quad 0002900040 \quad 00035 \quad 00040 \quad 0004400058 \quad 0006100048 \quad 00060000820010400130 \quad 00096$ $\begin{array}{lllllllllllllllll}0 & 028 & 00248 & 00225 & 00223 & 00873 & 00337 & 00516 & 00483 & 00179 & 00144 & 01687 & 00248 & 00294 & 00192 & 00024 & 00761\end{array}$

CRYSTAL HEIGHT $=03.000000$ IN. CRYSTAL RADIUS $=01.500000$ IN. $T=54027$ $I=10000$

INCIDENT ENERGY $=13.000000 \mathrm{MEV}$ SOURCE HEIGHT = 03.936992 IN. $000160001300016 \quad 000150002000012000120002000016000170001800015000170002400016 \quad 00021$ $00020000190001400023 \quad 00018 \quad 0001400020000210001500016 \quad 000190001900010000270001800023$ $0001400022 \quad 00023 \quad 00017 \quad 00026 \quad 0001400025 \quad 00017 \quad 00022000150001800017000200001900020 \quad 00028$ $000200002500019000220002300018 \quad 0002900029000360003000026 \quad 00040000290003800038 \quad 00033$ $\begin{array}{lllllllllllllllllll}00030 & 00030 & 00036 & 00026 & 00028 & 00027 & 00029 & 00025 & 00029 & 00028 & 00041 & 00027 & 00033 & 00029 & 00037 & 00030\end{array}$ $00033000350002900033 \quad 00032 \quad 00027 \quad 00038 \quad 00037 \quad 0003500025000390003800034000300003400032$ $0004100026 \quad 00036 \quad 00054 \quad 00042 \quad 00059000490004400046 \quad 00040 \quad 0004900060 \quad 00075 \quad 00102 \quad 00112 \quad 00146$ $0017800170 \quad 00368002910042200640 \quad 00428 \quad 007310042300300011350028500410002150006400607$ 
TABLE V (CONTINUED)

CRYSTAL HEIGHT $=05.000000 \mathrm{IN}$. CRYSTAL RADIUS $=02.500000 \mathrm{IN}$. $T=26783$ $I=10000$

001230002000031000350003000032000 $00040000370001500033 \quad 000240002900029$ 00019000200002200039000260003900031 $00030 \quad 000310002500028 \quad 00023 \quad 0002700032$ $0003400030 \quad 0002500024000330003300030$ $000320004400031000330004900047 \quad 00026$ 00010000040000300002000040000200002 00000000000000000000000000000000000
INCIDENT ENERGY $=00.661000$ MEV.
SOURCE HEIGHT $=03.936992$ IN. $A=06869$ $P=03958$ $000250003300024 \quad 00038 \quad 0004100036 \quad 00032 \quad 0003400036$ $\begin{array}{lllllllllll}0 & 0021 & 00034 & 00038 & 00033 & 00028 & 00034 & 00027 & 00033 & 00029\end{array}$

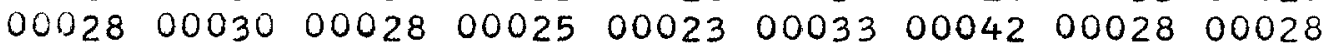
$\begin{array}{llllllllll}0 & 0032 & 00031 & 00028 & 00029 & 00030 & 00029 & 00030 & 00027 & 00030\end{array}$

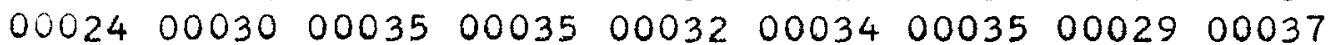
$\begin{array}{lllllllllll}0 & 0047 & 00052 & 00049 & 00052 & 00067 & 00031 & 00011 & 00010 & 00009\end{array}$ 000010000100000000000000000001000000000000000 000000000000000000000001300000000000000006869
CRYSTAL HEIGHT $=05.000000 \mathrm{IN}$. CRYSTAL RADIUS $=02.500000 \mathrm{IN}$. $T=30157$ $I=10000$ $00032000310003300032 \quad 000330003200034$ 00043000330003500031000280003700038 00036000410002900041000240003100046 $000280003200045000380003900050 \quad 00039$ $0005500048 \quad 0003900046000560005500051$ 00066000510006200051000710008300071 00006000060000300000000030000000001
INCIDENT ENERGY $=01.330000 \mathrm{MEV}$. $A=05267$ SOURCE HEIGHT

$=03.936992$ IN. $P=05924$ $\begin{array}{lllllllll}00035 & 00034 & 00037 & 00032 & 00038 & 00030 & 00044 & 00028 & 00039\end{array}$ $\begin{array}{llllllllll}00034 & 00031 & 00038 & 00031 & 00027 & 00036 & 00037 & 00035 & 00032\end{array}$ $\begin{array}{lllllllllll}0 & 0031 & 00027 & 00040 & 00034 & 00043 & 00035 & 00040 & 00029 & 00033\end{array}$ $\begin{array}{llllllllll}00052 & 00036 & 00041 & 00039 & 00041 & 00029 & 00029 & 00041 & 00032\end{array}$ $00036 \quad 00040 \quad 0004100046 \quad 0004500046 \quad 00043 \quad 0008600049$ 000490005400045000490006800061000510005100058 $\begin{array}{llllllllll}0 & 0086 & 00085 & 00080 & 00073 & 00054 & 00024 & 00026 & 00005 & 00014\end{array}$ 000000000000000000000000000000000090000005267
CRYSTAL HEIGHT $=05.000000 \mathrm{IN}$. CRYSTAL RADIUS $=02.500000 \mathrm{IN}$. $T=34824$

$I=10000$ $A=03941$

INCIDENT ENERGY $=02.620000 \mathrm{MEV}$ SOURCE HEIGHT = 03.936992 IN. $\begin{array}{lllllllllllllll} & 0 & 0 & 0\end{array}$ $00020 \quad 00038 \quad 0002700027 \quad 00035 \quad 00028 \quad 00034 \quad 00026 \quad 00032000330003100033000290003400030 \quad 00021$ $0002500016 \quad 00030 \quad 000190003500030 \quad 00030 \quad 00040 \quad 000300002900030 \quad 0002500029000410003600030$ $\begin{array}{lllllllllllllllll}00037 & 00031 & 00034 & 00031 & 00028 & 00039 & 00031 & 00036 & 00032 & 00033 & 00030 & 00038 & 00046 & 00035 & 00038 & 00027\end{array}$ $00037000410004700041000470003300038 \quad 000420002900037000350004900030 \quad 000330015900058$ $00056 \quad 00044000490005900061 \quad 00067 \quad 00050 \quad v 0 \cup 5900050 \quad 00058 \quad 000550006400053 \quad 00057 \quad 00068 \quad 00056$ $0005300064000670006400055000570008200563000870009800107 \quad 0009500095000940010200113$ $0012400104001230011400096 \quad 00037000370001800014000030000100005000030000300008 \quad 03941$ 
TABLE V (CONTINUED)

CRYSTAL HEIGHT $=05.000000$ IN. CRYSTAL RADIUS $=02.500000$ IN. $T=36604$

0001600021000210001400015000200002000019 0003100022000240002500021000220002500019 $00015 \quad 00025 \quad 0001900018 \quad 00014 \quad 000210002800014$ $\begin{array}{lllllllll}0 & 0028 & 00023 & 00032 & 00015 & 00028 & 00026 & 00020 & 00028\end{array}$ $0002700032000220002000036 \quad 000230003100029$ $\begin{array}{llllllll}00033 & 00036 & 00019 & 00026 & 00035 & 00036 & 00031 & 00042 \\ 00037 & 00041 & 00293 & 00089 & 00090 & 00073 & 00091 & 00098\end{array}$ $\begin{array}{llllllll}00037 & 00041 & 00293 & 00089 & 00090 & 00073 & 00091 & 00098 \\ 00088 & 01277 & 00171 & 00174 & 00166 & 00168 & 00158 & 00169\end{array}$

$$
\begin{array}{rrr}
\text { INCIDENT ENERGY } & =04.450000 \mathrm{MEV} \\
\text { SOURCE HEIGHT } & =03.936992 \mathrm{IN} \cdot \\
\mathrm{A}=03312 & \mathrm{P}=08942
\end{array}
$$
$\begin{array}{lllllllll}00023 & 00022 & 00019 & 00021 & 00022 & 00022 & 00017 & 00018\end{array}$ $\begin{array}{llllllll}00021 & 00027 & 00017 & 00029 & 00022 & 00025 & 00028 & 00021\end{array}$

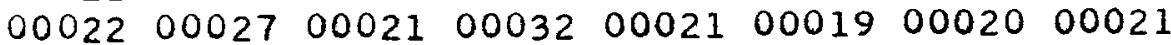
$\begin{array}{lllllllll}0 & 0026 & 00025 & 00019 & 00022 & 00023 & 00017 & 00036 & 00030\end{array}$ $\begin{array}{lllllllll}0 & 0033 & 00024 & 00033 & 00029 & 00030 & 00038 & 00029 & 00032\end{array}$ $00030 \quad 0002500037 \quad 00028 \quad 00041000470004100034$ $00096 \quad 00094 \quad 00094 \quad 00080 \quad 0009700073 \quad 00073 \quad 00068$ $001550007300096 \quad 0004700011000030000503319$

CRYSTAL HEIGHT $=05.000000$ IN. CRYSTAL RADIUS $=02.500000 \mathrm{IN}$. $T=36409$ $I=10000$ $A=02786$

INCIDENT ENERGY $=06.130000 \mathrm{MEV}$. SOURCE HEIGHT $=03.936992$ IN. 00012000090001900022000210001100010000170002100018000160002000016000150001500030

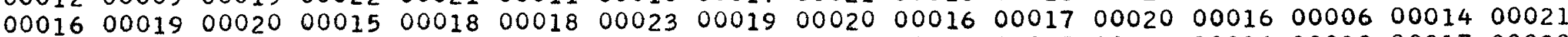
00017000190002600013000170002200017000210002400023000270001400016000180001700030

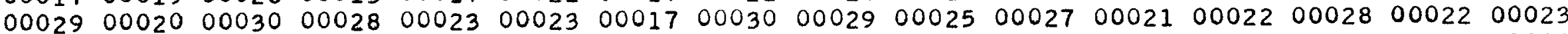
$000270002900021000200002100027 \quad 0002300016 \quad 00027000250002900018000250002100033 \quad 00026$

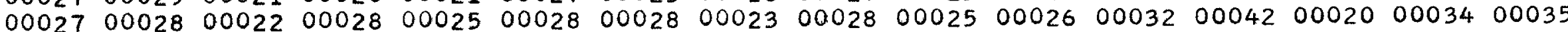

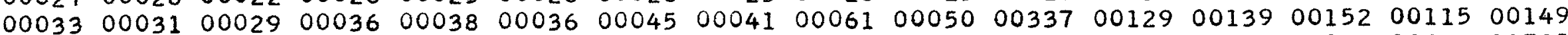
$0012200121000850011100086 \quad 0171800248 \quad 00240 \quad 0022700290 \quad 002340011000062000120001102795$

CRYSTAL HEIGHT $=05.000000 \mathrm{IN}$. CRYSTAL RADIUS $=02.500000 \mathrm{IN}$ $T=36173$ $I=10000$ 00010000120001100014000140001100014000130001800016000170001800016000100001300017 00023000190001200015000130001400021000160001900020000180001600014000100001400016 $00016 \quad 00013 \quad 00020 \quad 0001700006 \quad 00013 \quad 000100001700018000160001700018 \quad 00016000140001900015$ $0001400016 \quad 0001500021000200001900023000170002100025000210002200019000130002900021$ $00020 \quad 00021000220001700026 \quad 00022000190001300016 \quad 0001900030 \quad 0003200022000240002400024$

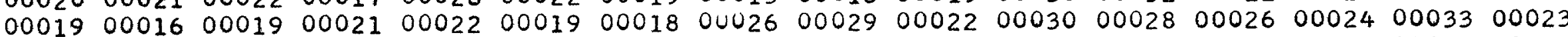
$0002700034 \quad 0003100026 \quad 00027 \quad 0002900030 \quad 00042 \quad 0002600037 \quad 00047 \quad 00057 \quad 00076 \quad 00098 \quad 00062 \quad 00371$ $001890021000220 \quad 00209002630016400116018990038900318002860029700149000750000702418$

INCIDENT ENERGY $=08.000000 \mathrm{MEV}$. SOURCE HEIGHT $=03.936992$ IN. $A=02406 \quad P=09065$ (1) 
TABLE V (CONTINUED)

CRYSTAL HEIGHT $=05.000000 \mathrm{IN}$ CRYSTAL RADIUS $=02.500000$ IN. $T=35307$ $I=10000$

$000180001400008 \quad 000070000600016000160000600005$ $00013 \quad 0001500013 \quad 000150001400021000150001000012$ $000070001600015000140001800016 \quad 000190002300022$ 000170002200012000200002400018000110002600019 $\begin{array}{llllllllll}00030 & 00040 & 00023 & 00017 & 00027 & 00026 & 00024 & 00015 & 00024 & 000 \\ 00021 & 00021 & 00021 & 00028 & 00030 & 00023 & 00014 & 00026 & 00024 & 00\end{array}$ $00028 \quad 00028 \quad 00032 \quad 00026 \quad 0002900030000330002900026 \quad 00$ 000790047200249002540020700154003140015001968

\section{INCIDENT ENERGY $=09.000000 \mathrm{MEV}$. SOURCE HEIGHT $=03.936992 \mathrm{IN}$. $A=02284$ $P=08734$} $00018 \quad 00016 \quad 00015 \quad 00010 \quad 00016 \quad 00016 \quad 00012$ $\begin{array}{lllllllll}00015 & 00016 & 00018 & 00017 & 00013 & 00009 & 00015\end{array}$ $\begin{array}{lllllllll}00007 & 00020 & 00014 & 00011 & 00012 & 00021 & 00018\end{array}$ $0001900011000240002300027 \quad 00013 \quad 00024$ $\begin{array}{llllllll}00019 & 00020 & 00017 & 00022 & 00019 & 00021 & 00025\end{array}$ 00033000240002300018000290002300023 $00030 \quad 00042 \quad 00045 \quad 00046 \quad 00051 \quad 0011300083$ $\begin{array}{llllllll}0 & 0431 & 00371 & 00299 & 00217 & 00122 & 00009 & 02290\end{array}$

CRYSTAL HEIGHT $=05.000000$ IN. CRYSTAL RADIUS $=02.500000$ IN. $T=35606$

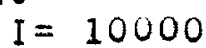

INCIDENT ENERGY $=11.000000 \mathrm{MEV}$ SOURCE HEIGHT $=03.936992$ IN. 00016000120000900006000070000700015000180000900007000140000900016000160001500012 $\begin{array}{lllllllllllllllllll}0 & 0016 & 00014 & 00017 & 00017 & 00015 & 00011 & 00013 & 00010 & 00009 & 00017 & 00016 & 00016 & 00012 & 00021 & 00021 & 00016\end{array}$ $00020 \quad 000200002100018 \quad 00013 \quad 00017000120001400012000210001800011000180001800016 \quad 00013$ 00017000170001500009000110001100020000170002000024000250002100018000260002700024 $000220002500025 \quad 00021 \quad 00024000190001900020000140001700026 \quad 00021000230002200013 \quad 00022$ $\begin{array}{lllllllllllllllll}00026 & 00018 & 00020 & 00022 & 00013 & 00030 & 00030 & 00017 & 00022 & 00020 & 00029 & 00025 & 00020 & 00028 & 00028 & 00024\end{array}$ $00031000350002400034 \quad 000250003200027 \quad 00043 \quad 0004400037000440004400076 \quad 000600008900088$ $\begin{array}{llllllllllllllll}00149 & 00166 & 00136 & 00177 & 00498 & 00230 & 00431 & 00379 & 00199 & 00152 & 02060 & 00392 & 00385 & 00250 & 00030 & 01935\end{array}$

CRYSTAL HEIGHT $=05.000000$ IN. CRYSTAL RADIUS $=02.500000 \mathrm{IN}$. $T=33861$ $1=10000$

7000100001500007 002000012000110001300013000150001500013

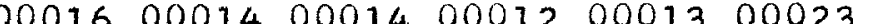
000090001800011000160001900011008 $\begin{array}{lllllll}00026 & 00021 & 00015 & 00011 & 00019 & 00031 & 006 \\ 00020 & 00014 & 00026 & 00024 & 00023 & 00027 & 000\end{array}$ $\begin{array}{lllllllllllllllllll}00019 & 00030 & 00028 & 00033 & 00032 & 00030 & 00034 & 00036 & 00037 & 00036 & 00032 & 00054 & 00053 & 00067 & 00065 & 00089\end{array}$ $0011600124002090018100269003480034500640 \quad 0042700352015090045800539003370007201682$
INCIDENT ENERGY $=13.000000 \mathrm{MEV}$. $A=01673$ $=03.936992$ IN. $P=08025$ 
CRYSTAL HEIGHT $=07.000000 \mathrm{IN}$. CRYSTAL RADIUS $=03.500000 \mathrm{IN}$. $T=20487$ $I=10000$

$0011400036 \quad 00035 \quad 00026 \quad 00022 \quad 00025 \quad 00028 \quad 00036$

$0001900016 \quad 000190002100025000230002500026$

$\begin{array}{lllllllll}00022 & 00019 & 00024 & 00031 & 00019 & 00020 & 00026 & 04022\end{array}$

$0002400015 \quad 000170002900024 \quad 000290001700017$

$\begin{array}{llllllll}00028 & 00025 & 00030 & 00022 & 00015 & 00033 & 00028 & 00032\end{array}$

$\begin{array}{lllllllll}00020 & 00039 & 00031 & 00048 & 00038 & 00041 & 00036 & 00031 \\ 00008 & 00004 & 00003 & 00004 & 00002 & 00001 & 00004 & 00001\end{array}$

$\begin{array}{lllllllll}00008 & 00004 & 00003 & 00004 & 00002 & 00001 & 00004 & 00001 \\ 00000 & 00000 & 00000 & 00000 & 00000 & 00000 & 00000 & 00000\end{array}$
INCIDENT ENERGY $=00.661000 \mathrm{MEV}$ SOURCE HEIGHT $=03.936992$ IN. $A=07379$ $P=02936$ $00033 \quad 00018 \quad 0003200018 \quad 00022000210002300022$ $0003500034 \quad 0002900025 \quad 0002500023 \quad 0001900023$ $\begin{array}{lllllllll}00021 & 00023 & 00025 & 00018 & 00018 & 00030 & 00018 & 00026\end{array}$ $\begin{array}{lllllllll}00015 & 00023 & 00014 & 00022 & 00020 & 00032 & 00018 & 00028\end{array}$ $\begin{array}{llllllllll}0 & 0036 & 00031 & 00023 & 00036 & 00034 & 00021 & 00027 & 00028\end{array}$ $\begin{array}{llllllllll}0 & 0 & 0 & 00041 & 00050 & 00051 & 00012 & 00011 & 00005 & 00012\end{array}$ 0000300000000000000000000000000000000000 $0000000000 \quad 000000001700000000000000007379$
CRYSTAL HEIGHT $=07.000000 \mathrm{IN}$ CRYSTAL RADIUS $=03.500000 \mathrm{IN}$. $T=22172$ $I=10000$

$00060 \quad 000250003500028 \quad 00028 \quad 00030 \quad 0002500025$ $\begin{array}{lllllllll}00028 & 00023 & 00033 & 00023 & 00029 & 00025 & 00023 & 00018\end{array}$ $00030 \quad 000250002700033000190003100026 \quad 00028$ $\begin{array}{llllllll}00034 & 00024 & 00027 & 00028 & 00029 & 00025 & 00041 & 00031\end{array}$ $\begin{array}{llllllll}00026 & 00025 & 00026 & 00028 & 00034 & 00029 & 00038 & 00043 \\ 00034 & 00044 & 00037 & 00032 & 00042 & 00034 & 00037 & 00051\end{array}$ $00043 \quad 00052 \quad 000550005300057000570008100057$ 0001100007000050000100000000000000000000
INCIDENT ENERGY $=01.330000 \mathrm{MEV}$. SOURCE HEIGHT $=03.936992$ IN. $A=06093$ 0002500023000270002900023000250002500019 $\begin{array}{llllllllll}00031 & 00024 & 00034 & 00025 & 00027 & 00040 & 00037 & 00027\end{array}$ $000150002900030 \quad 00028 \quad 0002000026 \quad 0002400036$ $\begin{array}{lllllllll}00030 & 00031 & 00026 & 00034 & 00026 & 00039 & 00028 & 00034\end{array}$ $\begin{array}{lllllllll}00031 & 00033 & 00031 & 00041 & 00036 & 00043 & 00063 & 00037\end{array}$ $00048 \quad 00061 \quad 00053 \quad 0004100040 \quad 000330004700056$ $00083000740007900053 \quad 00022000150001200015$ 0000000000000000000000000000080000006093
CRYSTAL HEIGHT $=07.000000 \mathrm{IN}$. CRYSTAL RADIUS $=03.500000 \mathrm{IN}$. $T=24658$ $I=10000$
INCIDENT ENERGY $=02.620000 \mathrm{MEV}$. $A=04969$

$=03.936992$ IN. $P=05921$

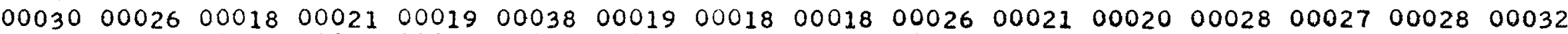
$00030 \quad 00019000190002500026 \quad 00019000270002400020000220002400030000310002600026 \quad 00032$ $0002500026 \quad 0001300020 \quad 000240001900022000250002100029000220002700026 \quad 0002400020 \quad 00024$ $000210003500025 \quad 0003500034 \quad 0002900026 \quad 000240002700031000340002500018 \quad 0002900037 \quad 00029$ $0002400026 \quad 00026 \quad 00024 \quad 00028 \quad 00029000310003200031000250003500035000220003900090 \quad 00048$ $0003800046 \quad 0003200036 \quad 000390004100030 \quad 0004700047 \quad 000450004700046 \quad 00048 \quad 000470004400038$ $\begin{array}{lllllllllllllllll}00054 & 00053 & 00051 & 00052 & 00050 & 00054 & 00057 & 00516 & 00086 & 00073 & 00074 & 00085 & 00089 & 00083 & 00102 & 00090\end{array}$ 00081001010010700114000950004400041000100001000004000030000100005000040001104969 
TABLE V (CONTINUED)

CRYSTAL HEIGHT $=07.000000$ IN. CRYSTAL RADIUS $=03.500000 \mathrm{IN}$. $T=25761$ $I=10000$

000120001600013000210001500015

0001400022000120002000018000280001500021

00018000230001800011000120001700019000110001900

$0001800013 \quad 0002200017000210002700015000220001700$

$\begin{array}{lllllllllll}00018 & 00018 & 00021 & 00019 & 00021 & 00019 & 00029 & 00017 & 00031 & 00\end{array}$

$\begin{array}{lllllllllll}00033 & 00032 & 00023 & 00025 & 00027 & 00030 & 00036 & 00019 & 00032 & 000\end{array}$

$000270003900178 \quad 00076 \quad 000680006600072$

00071012100017600162001680015400160

\begin{tabular}{ccccccc}
\multicolumn{4}{c}{ INCIDENT ENERGY } & $=04.450000$ & MEV. \\
SOURCE HEIGHT & $=03.936992$ & IN. \\
$A=$ & 04220 \\
15 & 00016 & 00019 & 00011 & 00022 & 00020 & 00016 \\
17 & 00025 & 00022 & 00020 & 00024 & 00020 & 00011 \\
19 & 00022 & 00021 & 00020 & 00021 & 00024 & 00023 \\
26 & 00019 & 00021 & 00029 & 00016 & 00021 & 00021 \\
26 & 00022 & 00033 & 00022 & 00018 & 00026 & 00021 \\
27 & 00028 & 00025 & 00033 & 00024 & 00026 & 00028 \\
72 & 00067 & 00083 & 00066 & 00069 & 00060 & 00056 \\
92 & 00079 & 00046 & 00013 & 00002 & 00003 & 04228
\end{tabular}

CRYSTAL HEIGHT $=07.000000$ IN. CRYSTAL RADIUS $=03.500000 \mathrm{IN}$. $T=25807$

$I=10000$

INCIDENT ENERGY $=06.130000 \mathrm{MEV}$. SOURCE HEIGHT $=03.936992$ IN.
$A=03738 \quad P=06809$

$00011000140002000015 \quad 00016 \quad 0001200017000120001600018000140001200011000090001500011$ $\begin{array}{lllllllllllllllll}0 & 00022 & 00018 & 00011 & 00018 & 00011 & 00017 & 00006 & 00015 & 00017 & 00024 & 00012 & 00012 & 00014 & 00017 & 00016 & 00013\end{array}$ $00011000130001700014000200002100016 \quad 0001600019000110002100020000220001600008 \quad 00013$ $\begin{array}{lllllllllllllllll}0 & 0015 & 00023 & 00012 & 00015 & 00015 & 00024 & 00020 & 00009 & 00029 & 00011 & 00016 & 00020 & 00012 & 00014 & 00026 & 00017\end{array}$ $00018 \quad 00015 \quad 0001500023 \quad 0002100028 \quad 000170002300017000270001500017000160001400018 \quad 00021$

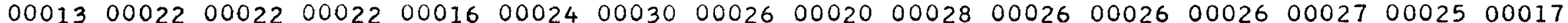
$00045 \quad 00034 \quad 00030 \quad 00028 \quad 00022 \quad 00026 \quad 0002400050 \quad 00036 \quad 000430023100100 \quad 00098 \quad 00078 \quad 00092 \quad 00100$ $00110 \quad 00096 \quad 00080 \quad 00090 \quad 00083 \quad 01644 \quad 00261002380022500285002350012000059000090000703744$

CRYSTAL HEIGHT $=07.000000$ IN. CRYSTAL RADIUS $=03.500000 \mathrm{IN}$. $T=25870$ $I=10000$

00007000060000900012000140001000013000070000900009000110001500013000090001300015 00014000100000600018000140001100012000100000900015000140001100019000190001100014 $0001400011 \quad 00010 \quad 00013000120001800009000090001600016 \quad 00016 \quad 0001200010000120001700021$ $0001300016 \quad 0001800018 \quad 00013 \quad 000250001400012000130002200021000090002200013 \quad 00016 \quad 00023$ $\begin{array}{llllllllllllllll}00026 & 00021 & 00016 & 00019 & 00015 & 00013 & 00020 & 00017 & 00015 & 00017 & 00018 & 00016 & 00018 & 00014 & 00020 & 00011\end{array}$ $0001900016 \quad 0001900028000120001300026000120002500012000230002300026000210002700029$ $\begin{array}{lllllllllllllllll}0 & 0023 & 00032 & 00027 & 00024 & 00029 & 00034 & 00029 & 00026 & 00030 & 00035 & 00045 & 00027 & 00053 & 00079 & 00048 & 00245\end{array}$ 00114001210015900179001940014100092018040040000323002760026600140000710000503459 $\begin{array}{ll}\text { INCIDENT ENERGY } & =08.000000 \mathrm{MEV} . \\ \text { SOURCE HEIGHT } & =03.936992 \text { IN. }\end{array}$ $3445 \quad P=06652$ 00114001210015900179001940014100092018040040000323002760026600140000710000503459 
TABLE $V$ (CONTTNUED)

CRYSTAL HEIGHT $=07.000000$ IN. CRYSTAL RADIUS $=03.500000 \mathrm{IN}$ $T=25298$

00010000080000600008000060000700008000090001100013000100001000013000160001300014 00014000150000900012000100001300015000110000900014000100002300015000140001100013 $00008 \quad 000070001500014000180001000014000130000900010000090001400011000120001500012$ $00012000120001100009000090002200016 \quad 00016 \quad 0001800014000160001800021000200001400017$ 00021000140001600019000190001900018000160001600013000220002400013000200001300017

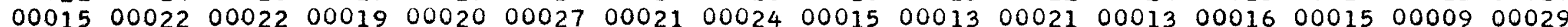

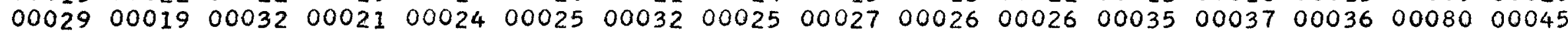
$00062002930016900170 \quad 001320016400241001640191900378003560032100224001130000503383$

CRYSTAL HEIGHT $=07.000000 \quad$ IN. CRYSTAL RADIUS $=03.500000 \mathrm{IN}$. $T=25231$ $I=10000$ 00010000080000500010000090001200010000030000600011000120000700011000090000900007 00011000130001400010000120001700011000110001000013000140001000012000120000900009 00010000090001100011000130001000005000160001200008000170001300012000170000500014 $000130001500016 \quad 00010 \quad 000120001300017000170001400013000180002000020000160001800012$ $0001900018 \quad 00020 \quad 0001700016 \quad 0002000016 \quad 0002000008000210001500015000130001600012 \quad 00021$

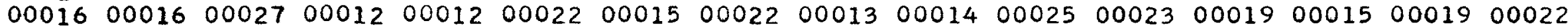
$000170001900025000220003700030 \quad 00030 \quad 000250002500033000370003900048000450006200072$ $\begin{array}{llllllllllllllll}00105 & 00125 & 00117 & 00147 & 00346 & 00174 & 00332 & 00336 & 00171 & 00149 & 01963 & 00413 & 00415 & 00249 & 00035 & 03044\end{array}$

CRYSTAL HEIGHT $=07.000000$ IN. CRYSTAL RADIUS $=03.500000 \mathrm{IN}$ $T=25077$ $I=10000$

INCIDENT ENERGY $=13.000000 \mathrm{MEV}$ SOURCE HEIGHT = 03.936992 IN. $00009000040000900011000060001100006 \quad 000090001300010000180000700014000100000700016$ 00009000070000900013000090001100011000040001500012000090000800011000120000700006 $00010 \quad 00014000090001200011000120001400012000090000700010 \quad 0001500015000140001500013$ $0001400022000100001300016 \quad 00010 \quad 00015000210001200015000140001000016000200001700012$ $0001200028 \quad 0000900013 \quad 000140001400013000180001100016000200001100022000180001400011$ $00016000170001400020 \quad 0002000025 \quad 00021000150001400016000190002100019000220001600013$

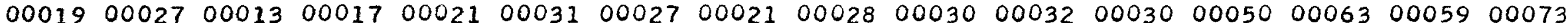
$00095000940014500147002160025100273 \quad 00460 \quad 0033700312014150051300595003820009402855$ 
TABLE VI

BROAD PARALLEL BEAM INCIDENT ON SPHERE OF NaI. SPHERE RADIUS IS 1.0 IN.

CRYSTAL HEIGHT $=02.000000$ IN. CRYSTAL RADIUS $=01.000000 \mathrm{IN}$. $I=10000$ 01010001200003000010000300001000040000300005 000040000400004000040000000007 000030000700006000090000400005008 $00000 \quad 00000 \quad 0000000000 \quad 00000 \quad 00000 \quad 004$ $\begin{array}{lllllll}00000 & 00000 & 00000 & 00000 & 00000 & 00000 \\ 00000 & 00000 & 00000 & 00000 & 00000 & 00000\end{array}$ $00000 \quad 0000000000000000000000000$ 000000000000000000000000000000
INCIDENT ENERGY $=00.142000$ MEV. SOURCE HEIGHT $=00.000000$ IN. $P=00113$ $\begin{array}{lllllllll} & 00000 & 00004 & 00004 & 00009 & 00008 & 00002\end{array}$ $00002000060000600003000040001000006 \quad 000040000600006$ $0000700006000100000900008 \quad 0001200017000090000100000$ $0000000000 \quad 00001000000000000000 \quad 00000000000000000000$ 00000000000000000000000000000000000000000000000000 $000000000000000 \quad 00000 \quad 00000 \quad 00000 \quad 00000 \quad 0000000000 \quad 00000$ $00000 \quad 00000 \quad 00000 \quad 00000 \quad 002610001100000 \quad 000000000000000$ 00000000000000000000000000000000000000000000009372
CRYSTAL HEIGHT $=02.000000$ IN. CRYSTAL RADIUS $=01.000000 \mathrm{IN}$. $I=10000$ $\begin{array}{llllllllllll} & 000035 & 00029 & 00032 & 00035 & 00033\end{array}$

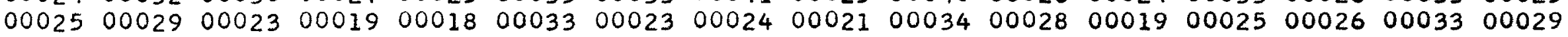
$\begin{array}{lllllllllllllllll}00028 & 00021 & 00032 & 00027 & 00027 & 00023 & 00015 & 00026 & 00017 & 00025 & 00021 & 00030 & 00023 & 00028 & 00036 & 00016\end{array}$ $\begin{array}{lllllllllllllllll}0 & 0038 & 00050 & 00037 & 00035 & 00035 & 00032 & 00042 & 00017 & 00008 & 00008 & 00007 & 00002 & 00001 & 00001 & 00002 & 00001\end{array}$ $000010000100002000020000000000 \quad 00002000010000000000000010000000000000010000000000$ $00000 \quad 00000 \quad 00000 \quad 00000 \quad 00000 \quad 00000 \quad 00000 \quad 00000 \quad 0000000000000000000000000 \quad 0000000000 \quad 00000$ 00000000000000000000000000000000069000000000000000000000000000000000000000007491

CRYSTAL HEIGHT $=02.000000$ IN. CRYSTAL RADIUS $=01.000000$ IN. $I=10000$ $0027400042000430005000054000650004600041 \quad 00053$ $0004400039000450004900046 \quad 00041000390004200045$ 000350003400041000390003600034000450003900037 $\begin{array}{lllllllll}00030 & 00036 & 00034 & 00041 & 00034 & 00030 & 00031 & 00038 & 00033 \\ 00027 & 00036 & 00039 & 00029 & 00036 & 00036 & 00039 & 00035 & 00052\end{array}$ $00006 \quad 00008 \quad 00007 \quad 00006 \quad 00007 \quad 00002 \quad 00006 \quad 00004 \quad 00003$ $\begin{array}{llllllllll}00001 & 00002 & 00000 & 00000 & 00001 & 00000 & 00000 & 00001 & 00000 \\ 00000 & 00000 & 00000 & 00000 & 00000 & 00000 & 00000 & 00000 & 00029\end{array}$

\begin{tabular}{|c|c|c|c|c|}
\hline $\begin{array}{l}\text { NCIDENT } \\
\text { OURCE }\end{array}$ & ENER & $\begin{array}{l}=00 \\
=00\end{array}$ & $\begin{array}{r}3200 \\
0000 \\
P\end{array}$ & $\begin{array}{r}\text { MEV. } \\
\text { IN. } \\
02245\end{array}$ \\
\hline 00035 & 00029 & 00032 & 00035 & 00033 \\
\hline 00024 & 00033 & 00026 & 00033 & 00025 \\
\hline 00019 & 00025 & 00 & 0 & 00029 \\
\hline 000 & 00023 & 000 & & 00016 \\
\hline $\begin{array}{l}00002 \\
00000\end{array}$ & $\begin{array}{l}00001 \\
00000\end{array}$ & $\begin{array}{l}00001 \\
00001\end{array}$ & $\begin{array}{l}00002 \\
00000\end{array}$ & $\begin{array}{l}00001 \\
00000\end{array}$ \\
\hline & 000 & 00000 & 00000 & \\
\hline & 00000 & 000 & 00000 & \\
\hline
\end{tabular}

INCIDENT ENERGY $=00.411000 \mathrm{MEV}$. SOURCE HEIGHT $=00.000000$ IN $P=03839$ 00048000460005100042000440003600049 $000320004700036 \quad 00043 \quad 0004200040 \quad 00031$ $00030 \quad 00046 \quad 0004500044000290003900041$ $0003100045000450004400038 \quad 00042 \quad 00046$ $00040 \quad 00045 \quad 000470003700046 \quad 0005100012$ $0000200003 \quad 0000100000000010000100000$ 00000000000000000000000000000000000 00000000000000000000000000000006445 
TABLE VI (CONTINUED)

CRYSTAL HEIGHT $=02.000000$ IN. CRYSTAL RADIUS $=01.000000 \mathrm{IN}$. $I=10000$

00293000490006100061000490004900061 00061000470005500047000560004900042 $\begin{array}{lllllllll}00051 & 00047 & 00057 & 00038 & 00040 & 00045 & 00038 & 006\end{array}$ $\begin{array}{lllllllll}00032 & 00052 & 00043 & 00042 & 00044 & 00054 & 00041 & 0 \\ 00050 & 00045 & 00046 & 00046 & 00048 & 00041 & 00040 & 004\end{array}$

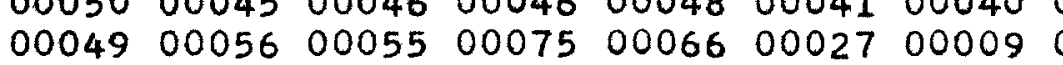
$\begin{array}{llllllll}00002 & 00001 & 00001 & 00003 & 00003 & 00000 & 00001 \\ 00000 & 00000 & 00000 & 00000 & 00000 & 00000 & 00000\end{array}$

CRYSTAL HEIGHT $=02.000000 \mathrm{IN}$. CRYSTAL RADIUS $=01.000000 \mathrm{IN}$. $I=10000$

$00221000660006500070 \quad 000630006600067000570006600059000560007100062000700004300047$ $\begin{array}{llllllllllllllllllll}00051 & 00058 & 00058 & 00054 & 00068 & 00057 & 00058 & 00052 & 00056 & 00061 & 00060 & 00056 & 00052 & 00047 & 00043 & 00059\end{array}$ $\begin{array}{lllllllllllllllll}0 & 0045 & 00057 & 00052 & 00060 & 00047 & 00055 & 00052 & 00055 & 00063 & 00052 & 00074 & 00047 & 00044 & 00046 & 00066 & 00063\end{array}$ $\begin{array}{lllllllllllllllllll}00052 & 00055 & 00051 & 00052 & 00044 & 00041 & 00047 & 0 & 0 & 058 & 00061 & 0.0051 & 00074 & 00058 & 00045 & 00046 & 00057 & 00054\end{array}$ $0005200055 \quad 000420006200060 \quad 000550004700050 \quad 0005500065000530006900060000590006400066$ $\begin{array}{llllllllllllllllllllll}00060 & 00066 & 00051 & 00071 & 00069 & 00058 & 00047 & 00082 & 00073 & 00077 & 00062 & 00075 & 00037 & 00013 & 00023 & 00011\end{array}$ 00012000080001000005000050000300004000040000400001000010000000000000000000000000 $00000 \quad 0000000000 \quad 00000 \quad 00000 \quad 00000 \quad 00000 \quad 000000000000000000000001600000000000000004375$

CRYSTAL HEIGHT $=02.000000$ IN. CRYSTAL RADIUS $=01.000000$ IN. $I=10000$

$00212000680006700058 \quad 0005300044000550006700053$

$00056 \quad 000650006000048000560006100063$ $0005200048 \quad 0006300056 \quad 000540007100060$ 00055000520004900063000460004600062 00063000490005600052000610006600070 00065000670007000073000600007900069 $\begin{array}{llllllll}00022 & 00015 & 00020 & 00010 & 00017 & 00013 & 00012 \\ 00000 & 00000 & 00000 & 00000 & 00000 & 00000 & 00000\end{array}$

INCIDENT ENERGY $=00.510000$ MEV。 SOURCE HEIGHT $=00.000000$ IN. $A=05397$ $\begin{array}{lllllllll}00062 & 00057 & 00055 & 00053 & 00063 & 00053 & 00050 & 00059 & 00049 \\ 00050 & 00060 & 00050 & 00041 & 00042 & 00054 & 00048 & 00054 & 00043\end{array}$ $\begin{array}{llllllllll}0 & 00054 & 00053 & 00038 & 00057 & 00042 & 00051 & 00054 & 00051\end{array}$ $\begin{array}{lllllllllll}041 & 00038 & 00049 & 00046 & 00040 & 00059 & 00039 & 00050 & 00048\end{array}$ $005400057 \quad 00053 \quad 00043 \quad 00052000490004600046 \quad 00053$ $110001000000 \quad 000020000700003000050001000010$ 000000000000001000000000000000000000000000000 20000000000000000000005397

INCIDENT ENERGY $=00.661000 \mathrm{MEV}$. SOURCE HEIGHT $=00.000000$ IN. $P=06969$ 
CRYSTAL HEIGHT $=02.000000$ IN. CRYSTAL RADIUS $=01.000000$ IN.

$I=10000$

000640006000064000530006600057000430005600061000720005900056000530005600058

$00054000710004800055000520005200063 \quad 000580005200044000460004000040000510005100059$

$00048 \quad 00056 \quad 0006800060 \quad 000550005900043 \quad 0004400051 \quad 000490006200066 \quad 000540005300045 \quad 00058$

$00053 \quad 0005400055000610006700040 \quad 00060 \quad 0005300052 \quad 00052 \quad 0005100065000690006000056 \quad 00054$

$\begin{array}{lllllllllllllllllll}00058 & 00058 & 00070 & 00060 & 00051 & 00061 & 00060 & 00060 & 00055 & 00056 & 00052 & 00063 & 00053 & 00077 & 00074 & 00089\end{array}$

$00070 \quad 0005900065 \quad 00072 \quad 00068 \quad 00070 \quad 00080 \quad 00088 \quad 0006400073 \quad 000790008700090 \quad 00078 \quad 0008900084$ $0009100090 \quad 0002500027000150001100011000150001100007000090000000004000030000300002$ $00000 \quad 000000000000000 \quad 00000000000000000000000000000000000000000024000000000003736$

CRYSTAL HEIGHT $=02.000000$ IN. CRYSTAL RADIUS $=01.000000$ IN. $I=10000$

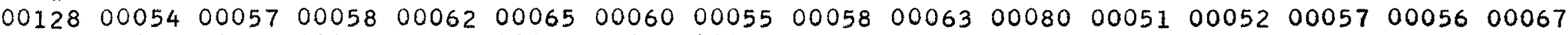

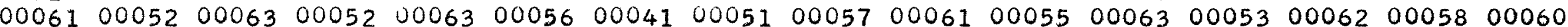
$000710005700050 \quad 00055 \quad 00051 \quad 00063 \quad 00054 \quad 00056 \quad 0004900053 \quad 00052 \quad 0005400058 \quad 000400006300062$ $00070 \quad 000540004300058 \quad 000570004200057 \quad 00056 \quad 000520005700040 \quad 0004700053 \quad 0005100058 \quad 00053$

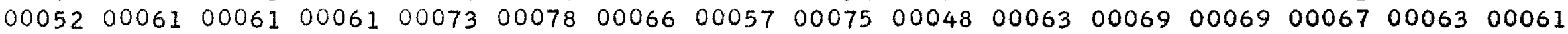
$000690007700075 \quad 00073 \quad 00074 \quad 00056 \quad 00074 \quad 00073 \quad 00077 \quad 0008100093 \quad 00076 \quad 000890009100088 \quad 00089$

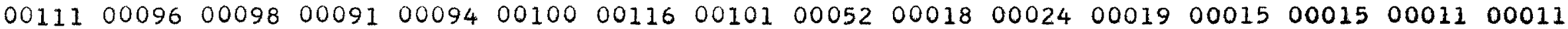
00003000020000500001000000000100000000000000000000000000000000000000110000003059

CRYSTAL HEIGHT $=02.000000$ IN. CRYSTAL RADIUS $=01.000000 \mathrm{IN}$. $I=10000$

00145000660005500062000670005700059000550003200052000510004500038000490005900057 $0005200048 \quad 00071 \quad 00054 \quad 0004700051 \quad 00055 \quad 000470006800080000610006300058000410007000054$ $000420005400057000520004500061 \quad 00042 \quad 000520004700066000570005700064000610006100065$ $0004500051 \quad 00051 \quad 00055 \quad 00050 \quad 00061 \quad 00056 \quad 00056 \quad 0004600054 \quad 00058 \quad 00054 \quad 00072000480005400055$ $0005500052000510006100058 \quad 0006100068000650005900068000720005900104000620006800069$ $000610006900086 \quad 00073 \quad 000670007700080 \quad 00066000610008000076 \quad 0008100080000970007100084$ $00100 \quad 00102 \quad 0009100094 \quad 00103 \quad 00107 \quad 00116 \quad 0011100112001160008700040 \quad 00026 \quad 0002400012 \quad 00016$ 00006000130000600003000000000100000000000000000000000000000000000000060000002838

INCIDENT ENERGY $=01.277000$ MEV. SOURCE HEIGHT $=00.000000$ IN. $P=11809$ 
TABLE VII

POINT SOURCE ON AXIS OF CSI CRYSTAL.

CRYSTAL HEIGHT $=00.500000$ IN. CRYSTAL. RADIUS $=00.250000$ IN. $I=10000$

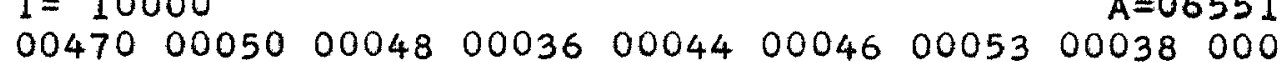

$00040 \quad 00042 \quad 00048 \quad 00047 \quad 000410003900040 \quad 00036 \quad 00038 \quad 00037000480004500041000380003500046$

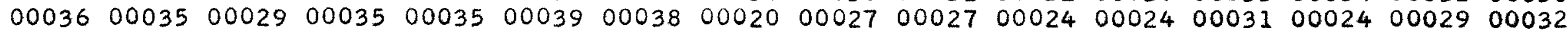

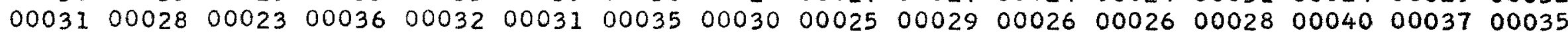
$0003700036 \quad 00023000030000100003 \quad 00003 \quad 000080000200002000000000200001000010000100002$ $0000000000 \quad 000000000100000 \quad 000000000100000 \quad 0000000000000000000000000 \quad 000000000000000$ $0000000000 \quad 00000 \quad 00000 \quad 00000 \quad 00000 \quad 00000 \quad 00000 \quad 0000000000000000000000000000000000000000$ 00000000000060600008000000000000000000000000000000000000000000000000000000006551

CRYSTAL HEIGHT $=00.500000$ IN. CRYSTAL RADIUS $=00.250000$ IN. $I=10000$

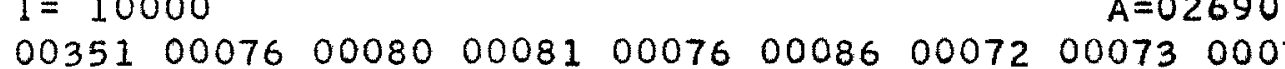

00067000740006800077000700006500081

$00068000620006900073 \quad 0006900050 \quad 00065$

$\begin{array}{lllllll}00063 & 00072 & 00064 & 00060 & 00075 & 00058 & 00048 \\ 00064 & 00069 & 00070 & 00072 & 00051 & 00063 & 00074\end{array}$

00081000770008700080000600009000118

$\begin{array}{lllllll}00014 & 00014 & 00010 & 00006 & 00004 & 00003 & 00004 \\ 00000 & 00000 & 00000 & 00000 & 00000 & 00000 & 00000\end{array}$

00000000000000000000000000000000000

CRYSTAL HEIGHT $=00.500000$ IN.

CRYSTAL RADIUS $=00.250000 \mathrm{IN}$. $I=10000$

$\begin{array}{llllllll}0 & 0133 & 00050 & 00062 & 00071 & 00071 & 00076 & 00078\end{array}$ $0007500076 \quad 0007100043000650006600062$ $\begin{array}{lllllllll}00056 & 00067 & 00064 & 00066 & 00064 & 00071 & 00065 & 006 \\ 00066 & 00063 & 00060 & 00075 & 00067 & 00066 & 00060 & 006\end{array}$ $00048 \quad 00066 \quad 000710007700050 \quad 00070 \quad 00054$ $0009400066 \quad 00078 \quad 00068 \quad 00102 \quad 0008800083$ $\begin{array}{lllllll}00087 & 00112 & 00117 & 00107 & 00133 & 00135 & 00132 \\ 00014 & 00014 & 00005 & 00005 & 00002 & 00002 & 00003\end{array}$
INCIDENT ENERGY $=00.279000$ MEV. SOURCE HEIGHT = 03.936992 IN. $P=05906$ INCIDENT ENERGY $=00.661000 \mathrm{MEV}$. SOURCE HEIGHT $=03.936992$ IN.

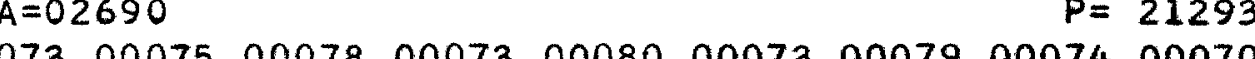
00790007400070 $00067000920006500080000640007200073 \quad 0006000078$ $\begin{array}{llllllllll}0 & 0052 & 00065 & 00062 & 00076 & 00067 & 00063 & 00067 & 00068 & 00052\end{array}$ $\begin{array}{llllllllll}00066 & 00059 & 00061 & 00064 & 00072 & 00056 & 00055 & 00077 & 00069\end{array}$ $\begin{array}{lllllllllll}0 & 0081 & 00066 & 00073 & 00066 & 00067 & 00096 & 00074 & 00066 & 00076\end{array}$ $\begin{array}{lllllllll}00118 & 00088 & 00116 & 00094 & 00098 & 00045 & 00020 & 00018 & 00013\end{array}$ $00004000020000100002000000000100000 \quad 0000000000$ $000000000000000002170000000000 \quad 00000 \quad 0000002690$ $\begin{array}{llllllllll}0 & 0137 & 00123 & 00122 & 00172 & 00076 & 00030 & 00033 & 00017 & 00016 \\ 0 & 0000 & 00000 & 00000 & 00000 & 00000 & 00000 & 00097 & 00000 & 01386\end{array}$ $\begin{array}{rl}A=01386 & P=35841\end{array}$ $00056 \quad 0006500069000620007400078 \quad 00048 \quad 00062 \quad 00077$ $\begin{array}{llllllllll}00059 & 00060 & 00060 & 00057 & 00056 & 00072 & 00122 & 00054 & 00071\end{array}$ $\begin{array}{llllllllll}00056 & 00076 & 00072 & 00048 & 00075 & 00077 & 00060 & 00065 & 00092\end{array}$ $\begin{array}{llllllllll}0 & 0066 & 00065 & 00071 & 00061 & 00054 & 00064 & 00065 & 00075 & 00072\end{array}$ $\begin{array}{lllllllllll}0 & 0067 & 00065 & 00079 & 00073 & 00067 & 00077 & 00070 & 00094 & 00087\end{array}$ $\begin{array}{llllllllll}0 & 0085 & 00100 & 00108 & 00097 & 00087 & 00105 & 00084 & 00105 & 00133\end{array}$ 
CRYSTAL HEIGHT $=00.500000$ IN. CRYSTAL RADIUS $=00.250000$ IN. $I=10000$ $000650004400046 \quad 000550006800053 \quad 00067$ $00064000660006400078 \quad 000690006700069$ 00068000730006600084000690007100061 $0006200066 \quad 0004500058 \quad 000670006100067$ $000760008400070 \quad 000780007200076 \quad 00066$ $000690007200080 \quad 00062000790008600073$ $001270013200178 \quad 0015500126 \quad 0003300028$
$A=00482$

INCIDENT ENERGY $=02.620000 \mathrm{MEV}$. SOURCE HEIGHT $=03.936992$ IN $P=46106$

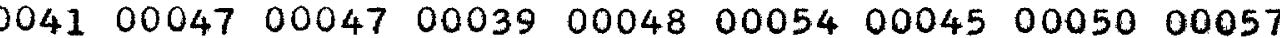
$00068 \quad 00045 \quad 00065 \quad 00058 \quad 00071 \quad 00062 \quad 00080 \quad 00060 \quad 00073$ $00070 \quad 0006900053 \quad 00078 \quad 00070 \quad 00070 \quad 00063 \quad 00064 \quad 00063$ $00050 \quad 00068 \quad 00064000650004900071 \quad 00072 \quad 0008500065$ $\begin{array}{lllllllllll}00068 & 00078 & 00055 & 00061 & 00069 & 00059 & 00070 & 00907 & 00088\end{array}$ $\begin{array}{llllllllll}00066 & 00079 & 00073 & 00068 & 00088 & 00093 & 00080 & 00084 & 00068\end{array}$ $\begin{array}{lllllllll}00262 & 00099 & 00111 & 00088 & 00090 & 00096 & 00108 & 00112 & 00125\end{array}$ $000120001300003 \quad 00003 \quad 000150000400008 \quad 0005200482$
CRYSTAL HEIGHT $=00.500000$ IN. CRYSTAL RADIUS $=00.250000$ IN. $I=10000$ 0003600037000430003800053 0006800076000550006400058006

$000580006400072000610006400058 \quad 00068$ 0005200063000720005200072000 0004200060000610006000053000780056000 000530014701839001300010200093 $00108 \quad 0040900097000790009400073$

INCIDENT ENERGY $=04.450000 \mathrm{MEV}$ SOURCE HEIGHT $=03.936992$ IN. $P=49773$

$A=00153$

$00028 \quad 0002600036 \quad 00043 \quad 00024000260003300040 \quad 00042$ $\begin{array}{llllllllll}00059 & 00059 & 00077 & 00056 & 00053 & 00049 & 00061 & 00083 & 00066\end{array}$ $\begin{array}{llllllllll}00053 & 00068 & 00068 & 00075 & 00068 & 00048 & 00055 & 00065 & 00067\end{array}$ $\begin{array}{llllllllll}00056 & 00059 & 00057 & 00061 & 00060 & 00073 & 00065 & 00079 & 00061\end{array}$ $\begin{array}{lllllllll}00056 & 00068 & 00074 & 00049 & 00068 & 00059 & 00063 & 00059 & 00066\end{array}$ $\begin{array}{llllllllll}00060 & 00057 & 00069 & 00052 & 00049 & 00056 & 00048 & 00074 & 00044\end{array}$ $\begin{array}{lllllllllll}0 & 0092 & 00094 & 00130 & 00118 & 00104 & 00083 & 00075 & 00068 & 00058\end{array}$

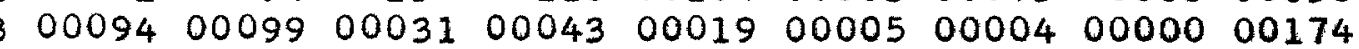

CRYSTAL HEIGHT $=00.500000 \mathrm{IN}$. CRYSTAL RADIUS $=00.250000 \mathrm{IN}$. $I=10000$ $000270001500008 \quad 000230001900017000250001900017$ $00034000270003400026 \quad 0003300026 \quad 00042 \quad 004$ 00083000550006100064000580005900068 $00049000580006600051000800006800082 \quad 006$ 00060000620006400079000600008400074 $\begin{array}{llllllll}00069 & 00057 & 00045 & 00070 & 00056 & 00061 & 00049 \\ 00053 & 00057 & 00056 & 00072 & 00063 & 00068 & 00061 & 000\end{array}$ 00141001220008400080001060045200055
INCIDENT ENERGY $=06.130000 \mathrm{MEV}$. SOURCE HEIGHT $=03.936992$ IN. $P=49256$

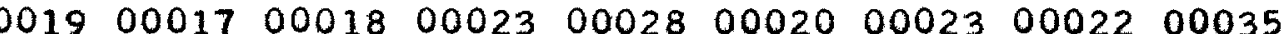
$00050 \quad 000440004100050 \quad 00063 \quad 0005500050 \quad 0004900059$ $\begin{array}{lllllllllll}00058 & 00063 & 00054 & 00062 & 00051 & 00057 & 00072 & 00064 & 00071\end{array}$ $\begin{array}{lllllllllll}0 & 00057 & 00061 & 00054 & 00054 & 00054 & 00060 & 00050 & 00062 & 00053\end{array}$ $0006100079000650005900049000550006300056 \quad 00055$ $\begin{array}{lllllllllll}0 & 0043 & 00053 & 00072 & 00064 & 00054 & 00060 & 00061 & 00065 & 00055\end{array}$ $\begin{array}{llllllllll}00106 & 00266 & 00083 & 02157 & 00163 & 00133 & 00142 & 00140 & 00137\end{array}$ $0003100060 \quad 00054000440001200009000050000400044$ 
TABLE VII (CONTINUED)

CRYSTAL HEIGHT $=01.000000$ IN. CRYSTAL RADIUS $=00.500000 \mathrm{IN}$. $I=10000$

0032200042000270002700041 000210001400017000150001300028 000190001700014000160002400020 000200003200027000020000600002 000000000000000000000000000000 000000000000000000000000000000 000000000000344000020000100000
INCIDENT ENERGY $=00.279000 \mathrm{MEV}$. SOURCE HEIGHT $=03.936992$ IN. $A=07785$ $P=02714$ $\begin{array}{llllllllll}00026 & 00034 & 00027 & 00029 & 00025 & 00035 & 00031 & 00020 & 00028 & 00026\end{array}$ $\begin{array}{lllllllllll}00019 & 00026 & 00024 & 00024 & 00027 & 00028 & 00016 & 00020 & 00021 & 00025\end{array}$ $\begin{array}{llllllllllll}00028 & 00018 & 00026 & 00016 & 00011 & 00020 & 00012 & 00016 & 00014 & 00016\end{array}$ $\begin{array}{lllllllllll}00014 & 00023 & 00018 & 00028 & 00019 & 00023 & 00021 & 00018 & 00030 & 00028\end{array}$ 00001000010000100001000000000200002000000000000000 $00000 \quad 00000 \quad 00000 \quad 00000 \quad 00000 \quad 00000 \quad 00000 \quad 00000 \quad 00000 \quad 00000$ $00000 \quad 00000 \quad 00000 \quad 00000 \quad 00000 \quad 00000 \quad 00000 \quad 00000 \quad 00000 \quad 00000$ $0000000000000000000000000 \quad 00000 \quad 00000000000000007785$
CRYSTAL HEIGHT $=01.000000$ IN. CRYSTAL RADIUS $=00.500000 \mathrm{IN}$. $I=10000$

$003140006700060 \quad 00071000730007200046 \quad 00048 \quad 00065$ 000520005300062000750007500048 000570006200053000650006500062 $0005800065000530004600048 \quad 00058$ 000510006200062000800005800059 $\begin{array}{lllllll}00064 & 00055 & 00067 & 00066 & 00067 & 00072 \\ 00015 & 00007 & 00012 & 00007 & 00004 & 00005\end{array}$ 000000000000000000000000000000
INCIDENT ENERGY $=00.661000 \mathrm{MEV}$ SOURCE HEIGHT $=03.936992$ IN.

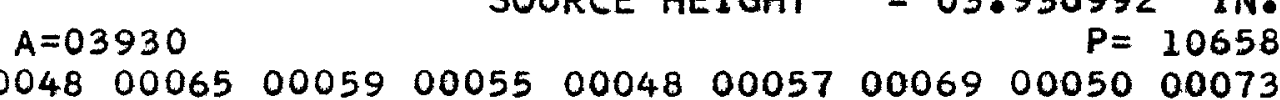
$\begin{array}{llllllllllll}0 & 0062 & 00058 & 00066 & 00050 & 00060 & 00049 & 00054 & 00055 & 00045 & 00058\end{array}$ $\begin{array}{lllllllllll}00063 & 00053 & 00056 & 00065 & 00072 & 00058 & 00059 & 00051 & 00062 & 00049\end{array}$ $\begin{array}{lllllllllll}00042 & 00045 & 00049 & 00051 & 00064 & 00057 & 00048 & 00052 & 00050 & 00057\end{array}$ $\begin{array}{lllllllllll}00052 & 00063 & 00051 & 00076 & 00067 & 00072 & 00070 & 00071 & 00055 & 00066\end{array}$ $\begin{array}{llllllllllll}0 & 0065 & 00073 & 00081 & 00080 & 00079 & 00065 & 00032 & 00014 & 00011 & 00008\end{array}$ 00004000020000400002000000000000000000000000000000 $00000 \quad 00000 \quad 00000 \quad 00000 \quad 00150 \quad 00000 \quad 00000 \quad 00000 \quad 0000003930$
CRYSTAL HEIGHT $=01.000000 \mathrm{IN}$. CRYSTAL RADIUS $=00.500000 \mathrm{IN}$. $I=10000$

$001280005400056 \quad 000600005800064000570006300063$ 000540006300056000590005000067 000550005500067000620005600057 $0006900058 \quad 00067 \quad 00056 \quad 00054 \quad 00063 \quad 0006900054 \quad 00062$ 000680005900066000630005400069000 $0008500073 \quad 00072000820007700077$ 000130000400006000060000400001
INCIDENT ENERGY $=01.330 .000 \mathrm{MEV}$. SOURCE HEIGHT $=03.936992$ IN. $P=18363$ $\begin{array}{llllllllll} & 00066 & 00057 & 00048 & 00057 & 00049 & 00048 & 00073\end{array}$ $\begin{array}{lllllllllll}00053 & 00060 & 00058 & 00064 & 00045 & 00056 & 00060 & 00089 & 00046 & 00062\end{array}$ $\begin{array}{lllllllllll} & 000068\end{array}$ $\begin{array}{lllllllllll}00076 & 00087 & 00069 & 00089 & 00090 & 00091 & 00098 & 00076 & 00081 & 00097\end{array}$

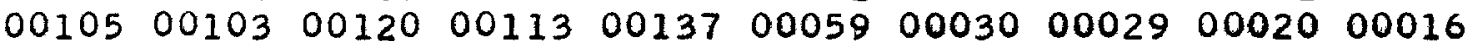
$00000000010000000000000000000100000 \quad 000720000102354$ 
CRYSTAL HEIGHT $=01.000000$ IN. CRYSTAL RADIUS $=00.500000$ IN.

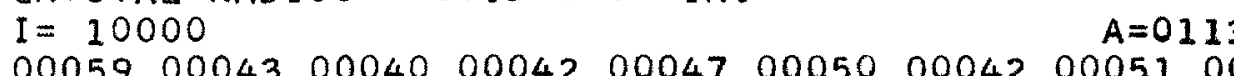

000390004300040000420004700050000420005100044

$0006100050 \quad 0005100057000430004900040 \quad 0004600053$

$00051000550004900051000590007000056 \quad 0006300062$

$0005100064000540006200060 \quad 0006900043 \quad 00040 \quad 00058 \quad 006$

$00080 \quad 00077 \quad 00060 \quad 00073 \quad 0008500061 \quad 00076 \quad 0008400068$

000810006200075000820007900096000880046100095

0013500161001420014700129
INCIDENT ENERGY $=02.620000 \mathrm{MEV}$ SOURCE HEIGHT $=03.936992 \quad \mathrm{IN}$. $\begin{array}{rr}P= & 24580 \\ 0043 & 00036\end{array}$ $\begin{array}{llllllll}00046 & 00049 & 00054 & 00040 & 00043 & 00043 & 00036\end{array}$ $00062000550004500056 \quad 0005900057 \quad 00060$ 00062000640005700049000520006400048 $000540006300050 \quad 0006000050 \quad 0064500079$ 00081000910008600079000910008400080 $\begin{array}{llllllll}00107 & 00110 & 00098 & 00124 & 00112 & 00138 & 00153\end{array}$ 00001000050000900001000040005901137
CRYSTAL HEIGHT $=01.000000$ IN. CRYSTAL RADIUS $=00.500000 \mathrm{IN}$. $I=10000$

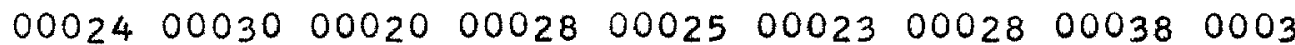

000440002900024000320004600

$0004900050 \quad 0003300047000400003100044$

0004100056000410005800047

0004100055000440003600058

0005100043000580005500049

$00050 \quad 00108 \quad 014340014900163$

0011900883001240011700105

$A=00589$
INCIDENT ENERGY = SOURCE HEIGHT

$=04.450000 \mathrm{MEV}$. $=03.936992 \quad I N$.

$P=26530$

$\begin{array}{llllllllllll}00023 & 00028 & 00038 & 00030 & 00025 & 00028 & 00021 & 00035 & 00029 & 00024 & 00038\end{array}$ $\begin{array}{llllllllllll}0 & 0038 & 00037 & 00029 & 00031 & 00032 & 00039 & 00043 & 00033 & 00041\end{array}$ $\begin{array}{llllllllllll} & 00004\end{array}$ $\begin{array}{lllllllllll}00038 & 00048 & 00044 & 00047 & 00051 & 00040 & 00048 & 00052 & 00062 & 00049 & 00044\end{array}$ $0004500043 \quad 0006500053 \quad 00055 \quad 00056 \quad 00054 \quad 0004800050 \quad 0005500051$ $\begin{array}{llllllllllll}00058 & 00044 & 00054 & 00055 & 00059 & 00061 & 00062 & 00035 & 00045 & 00043 & 00050\end{array}$ $\begin{array}{llllllllllll}0 & 0141 & 00151 & 00116 & 00147 & 00154 & 00126 & 00115 & 00115 & 00094 & 00070 & 00080\end{array}$ $\begin{array}{lllllllllllll}00115 & 00108 & 00123 & 00131 & 00053 & 00097 & 00041 & 00004 & 00005 & 00003 & 00626\end{array}$
CRYSTAL HEIGHT $=01.000000$ IN. CRYSTAL RADIUS $=00.500000$ IN. $I=10000$

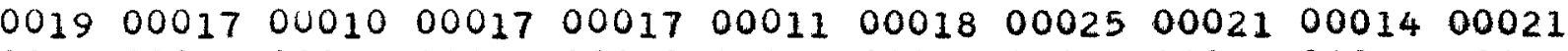
$\begin{array}{llllllllllllllllll}0 & 0 & 0 & 0018 & 00025 & 00021 & 00026 & 00028 & 00032 & 00030 & 00034 & 00030 & 00027 & 00031 & 00026 & 00031 & 00032 & 00028\end{array}$ $0004200040 \quad 00033 \quad 00038 \quad 00022 \quad 00034000420003700039000310004700028000420004200033 \quad 00043$ $\begin{array}{llllllllllllllllll}00045 & 00044 & 00054 & 00043 & 00033 & 00046 & 00031 & 00048 & 00035 & 00043 & 00034 & 00033 & 00036 & 00047 & 00048 & 00037\end{array}$ $000490004500043 \quad 00057 \quad 00051 \quad 00053 \quad 00039000430005900046000420004500041000420004500043$ $00046 \quad 00060 \quad 00052 \quad 00052 \quad 0004500052 \quad 00043 \quad 0005400050 \quad 000490004400047 \quad 00043 \quad 00046 \quad 00043 \quad 00055$

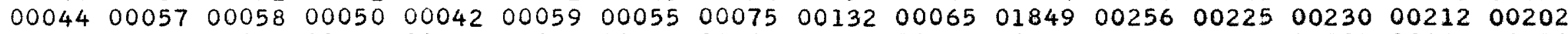

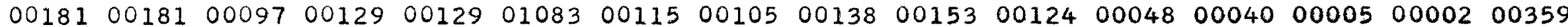

INCIDENT ENERGY $=06 \cdot 130000$ MEV SOURCE HEIGHT = 03.936992 IN. $P=25911$ 
CRYSTAL HEIGHT $=02.000000$ IN. CRYSTAL RADIUS $=01.000000$ IN. $I=10000$

$\begin{array}{lllll}00171 & 00019 & 00019 & 00021 & 00013\end{array}$ 0000900016000150001600012

0001200010000140001200008 0000700013000130001700011

0002500018000140000100000 $\begin{array}{llllll}00000 & 00001 & 00001 & 00000 & 00000 \\ 00000 & 00000 & 00000 & 00000 & 00000\end{array}$

$0000000000 \quad 002480000200000$
$A=08648$

0001700015 0001300011 0001300008 0001500017 0000400003 0000000000 0000000000 0000000000 $\begin{array}{lllllllll}A & 00014 & 00015 & 00014 & 00023 & 00014 & 00015 & 00017 & 00017\end{array}$ $00014000140000900010 \quad 0001300011000130001000008$ 000150001700007000090000900003000090001200012 $\begin{array}{llllllllll}00024 & 00019 & 00014 & 00013 & 00014 & 00016 & 00023 & 00017 & 00021\end{array}$ 000000000000000000010000000001000010000000000 $00000 \quad 00000 \quad 00000 \quad 00000 \quad 00000 \quad 00000 \quad 00000 \quad 00000 \quad 00000$ $00000 \quad 0000000000 \quad 00000 \quad 00000 \quad 00000 \quad 00000 \quad 0000000000$ $00000 \quad 00000 \quad 0000000000 \quad 00000 \quad 00000 \quad 00000 \quad 0000008648$

CRYSTAL HEIGHT $=02.000000 \mathrm{IN}$. CRYSTAL RADIUS $=01.000000 \mathrm{IN}$. $I=10000$

0024200053000420004900042

$000490005500043 \quad 000480004500$

$0003000050 \quad 000390004000049$

0004400051000330004400050

0004100035000430004100038

0005700045000570004600033

0001300005000040000800002

0000000000000000000000000
$A=05464$

$\begin{array}{lllllllllll}00047 & 00036 & 00049 & 00043 & 00048 & 00053 & 00051 & 00043 & 00042 & 00040 & 00046\end{array}$ $\begin{array}{lllllllllll}00029 & 00048 & 00044 & 00054 & 00042 & 00035 & 00035 & 00048 & 00048 & 00044 & 00065\end{array}$ $\begin{array}{lllllllllllll}0 & 0035 & 00046 & 00036 & 00037 & 00047 & 00038 & 00034 & 00041 & 00041 & 00032 & 00048\end{array}$ $\begin{array}{lllllllllllll}00039 & 00045 & 00039 & 00040 & 00050 & 00048 & 00041 & 00043 & 00034 & 00038 & 00043\end{array}$ $\begin{array}{llllllllllll}00042 & 00045 & 00047 & 00036 & 00045 & 00035 & 00031 & 00046 & 00047 & 00037 & 00041\end{array}$

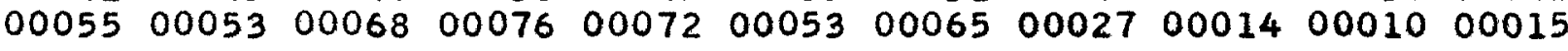
$0000100003000030000200000 \quad 00000 \quad 00000 \quad 00000000000000000000$ 0000000000000000000000000001160000000000000000000005464
CRYSTAL HEIGHT $=02.000000 \mathrm{IN}$. CRYSTAL RADIUS $=01.000000 \mathrm{IN}$. $I=10000$

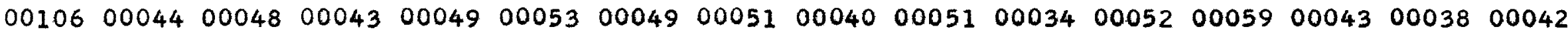
$00049000640004300037000420003900048 \quad 000440004400056 \quad 000360004200048000660003700053$

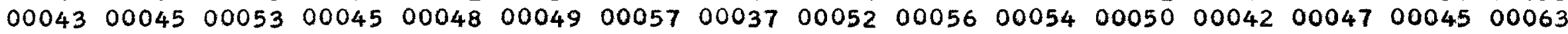
$\begin{array}{lllllllllllllllll}0 & 0048 & 00041 & 00048 & 00048 & 00048 & 00051 & 00055 & 00054 & 00041 & 00052 & 00054 & 00044 & 00058 & 00043 & 00061 & 00064\end{array}$ $\begin{array}{lllllllllllllllll}0 & 0052 & 00055 & 00051 & 00054 & 00055 & 00050 & 00048 & 00063 & 00047 & 00067 & 00053 & 00064 & 00070 & 00057 & 00079 & 00064\end{array}$ $\begin{array}{lllllllllllllllll}0 & 0048 & 00060 & 00074 & 00069 & 00068 & 00074 & 00063 & 00062 & 00077 & 00083 & 00071 & 00081 & 00074 & 00050 & 00069 & 00073\end{array}$ $\begin{array}{lllllllllllllllll}00077 & 00073 & 00078 & 00086 & 00082 & 00077 & 00081 & 00097 & 00074 & 00098 & 00093 & 00041 & 00024 & 00019 & 00017 & 00009\end{array}$ 00009000070000300003000000000000000000010000000000000000000000000000540000003726

INCIDENT ENERGY $=01.330000 \mathrm{MEV}$. SOURCE HEIGHT = 03.936992 IN. $\mathrm{P}=10152$

INCIDENT ENERGY $=00.661000 \mathrm{MEV}$. SOURCE HEIGHT $=03.936992$ IN. . 
CRYSTAL HEIGHT $=02.000000$ IN. CRYSTAL RADIUS $=01.000000 \mathrm{IN}$. $I=10000$ $00030 \quad 00033 \quad 0003200029000250003700030 \quad 0003500030$ $00050 \quad 00041000510003700036 \quad 0004700040 \quad 0003500034$

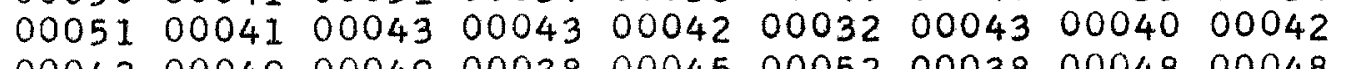
$\begin{array}{lllllllll}00042 & 00049 & 00049 & 00038 & 00045 & 00052 & 00038 & 00048 & 00048 \\ 00086 & 00071 & 00081 & 00068 & 00069 & 00072 & 00082 & 00078 & 00068\end{array}$ $\begin{array}{llllllllll}00086 & 00071 & 00081 & 00068 & 00069 & 00072 & 00082 & 00078 & 00068 \\ 00083 & 00062 & 00069 & 00082 & 00082 & 00081 & 00091 & 00682 & 00097\end{array}$ $00127001170013900113 \quad 00078 \quad 00047 \quad 00038 \quad 00020 \quad 00015$
INCIDENT ENERGY $=02.620000 \mathrm{MEV}$. SOURCE HEIGHT $=03.936992$ IN. $P=13739$ 00034000390003000035000370002400033 00038000440004400039000480004100032 $00046000450004400053 \quad 000430004200040$ $\begin{array}{lllllllll}0 & 0039 & 00035 & 00045 & 00048 & 00038 & 00047 & 00033\end{array}$ $\begin{array}{lllllllll}0 & 0039 & 00053 & 00051 & 00051 & 00048 & 00365 & 00061\end{array}$ $\begin{array}{lllllllll}0 & 0065 & 00078 & 00070 & 00072 & 00095 & 00088 & 00062\end{array}$ $\begin{array}{llllllll}00106 & 00113 & 00084 & 00137 & 00084 & 00132 & 00122\end{array}$ 00003000030000300004000030004402368
CRYSTAL HEIGHT $=02.000000 \mathrm{IN}$. CRYSTAL RADIUS $=01.000000$ IN. $I=10000$

000140002700023000230001700014000290002400019 $00026 \quad 00026 \quad 00023 \quad 00022 \quad 00026 \quad 00026 \quad 00027 \quad 0003300026$ $0002400026 \quad 00016 \quad 00042 \quad 00027 \quad 00024000380002100037$ $\begin{array}{lllllllll}00033 & 00043 & 00039 & 00027 & 00041 & 00034 & 00036 & 00033 & 00047 \\ 00039 & 00025 & 00043 & 00038 & 00046 & 00034 & 00047 & 00040 & 00052\end{array}$ $\begin{array}{lllllllll}00039 & 00025 & 00043 & 00038 & 00046 & 00034 & 00047 & 00040 & 00052 \\ 00040 & 00038 & 00046 & 00051 & 00046 & 00046 & 00047 & 00038 & 00043\end{array}$

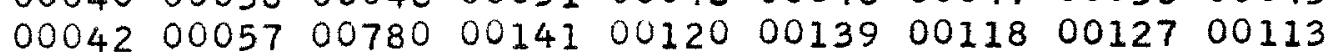
$0011101396 \quad 00156001360015500143001590016000141$
INCIDENT ENERGY $=04.450000 \mathrm{MEV}$. SOURCE HEIGHT $=03.936992$ IN.

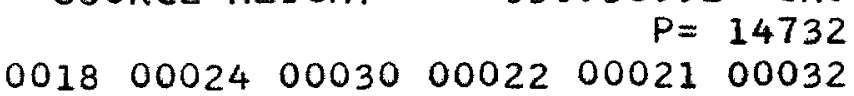
$0003300026 \quad 00030 \quad 000230002500036$ 000260003200025000300003500038 000340003500038000450004600033 000450004900043000450003300044 $00046000320003500050 \quad 0004700058$ 001150013600095000710009400098 000930006200008000010000501612
CRYSTAL HEIGHT $=02.000000$ IN. CRYSTAL RADIUS $=01.000000$ IN. $I=10000$ 000170001000015000170001200016000180001800014 $000110001900020 \quad 00017 \quad 00014 \quad 0001900013 \quad 0001700016 \quad 00016 \quad 00022$ $0001900025 \quad 00018 \quad 00022 \quad 00034 \quad 000200003100028000170002200033$

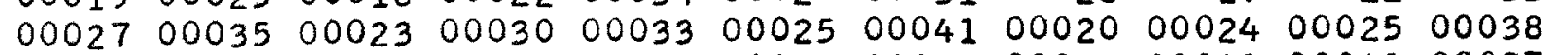
$000340003200033 \quad 00031000330003400032 \quad 00043000310004100027$

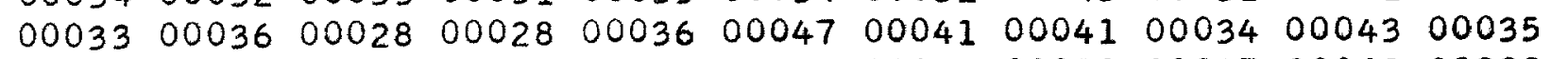
$0004400053 \quad 00053 \quad 00043 \quad 00060 \quad 000450004300050 \quad 000870006200989$ $\begin{array}{lllllllllll}00172 & 00155 & 00110 & 00111 & 00132 & 01877 & 00188 & 00163 & 00214 & 00240 & 00167\end{array}$
INCIDENT ENERGY $=06.130000 \mathrm{MEV}$ SOURCE HEIGHT $=03.936992$ IN. $P=14366$ $\begin{array}{lllll}00020 & 00022 & 00019 & 00010 & 00019 \\ 00022 & 00018 & 00017 & 00024 & 00014\end{array}$ 0002400020000370003200025 $000270002500028 \quad 00028 \quad 00035$ $\begin{array}{llllll}00042 & 00047 & 00038 & 00038 & 00038\end{array}$ $00030 \quad 00046000420005600041$ $\begin{array}{llllll}00218 & 00228 & 00197 & 00203 & 00177\end{array}$ 0008300042000020000501188 
CRYSTAL HEIGHT $=03.000000 \mathrm{IN}$. CRYSTAL RADIUS $=01.500000$ IN. $I=10000$ 0000500009000080001400010 0000700013000130000700009000090 00007000070001100006000090000500008 00022000170001700003000000000400002 00000000000000000000000000000000000 $00000 \quad 00000 \quad 00000 \quad 0000000000 \quad 00000 \quad 00000$ 00000000000020700002000000000000000

\section{$A=08898$}

INCIDENT ENERGY $=00.279000 \mathrm{MEV}$ SOURCE HEIGHT = 03.936992 IN. $P=02725$ $0000700008 \quad 0001400013000170001100008 \quad 0001300014$ $00010 \quad 00003 \quad 00007 \quad 00006 \quad 00003 \quad 00008 \quad 000150001500006$ $0001500016 \quad 00010 \quad 00018 \quad 0001100014000320002700022$ $0000100000 \quad 00002000020000100000 \quad 000000000100000$ $00000 \quad 00000 \quad 00000 \quad 00000 \quad 00000 \quad 00000 \quad 00000 \quad 00000 \quad 00000$ $00000 \quad 00000 \quad 00000 \quad 00000 \quad 00000 \quad 00000 \quad 00000 \quad 00000 \quad 00000$ $0000000000 \quad 00000 \quad 0000000000 \quad 00000 \quad 00000 \quad 0000008898$
CRYSTAL HEIGHT $=03.000000$ IN. CRYSTAL RADIUS $=01.500000 \mathrm{IN}$. $I=10000$

$0017600036 \quad 00028 \quad 00027 \quad 00034 \quad 0004100040 \quad 00029000$ $000330003400026 \quad 000420003300036$ $00032000470002800040 \quad 0002700026$ $0003500043 \quad 00035000280003700038$ $000400002800030 \quad 000300003600031$ $\begin{array}{llllll}00041 & 00030 & 00029 & 00035 & 00040 & 00044 \\ 00006 & 00005 & 00009 & 00006 & 00002 & 00002\end{array}$ 000000000000000000000000000000
INCIDENT ENERGY $=00.661000 \mathrm{MEV}$. SOURCE HEIGHT

$=03.936992 \quad 1 \mathrm{~N}$. $P=04736$ 00033000270003800042000380002900031000410002700042 $\begin{array}{llllllllllll}00033 & 00022 & 00043 & 00020 & 00039 & 00041 & 00025 & 00031 & 00052 & 00038\end{array}$ $\begin{array}{llllllllll}00026 & 00026 & 00023 & 00022 & 00029 & 00037 & 00035 & 00031 & 00039 & 00025\end{array}$ $\begin{array}{llllllllll}00036 & 00023 & 00033 & 00032 & 00036 & 00028 & 00028 & 00033 & 00035 & 00027\end{array}$ $\begin{array}{llllllllllll}0 & 0036 & 00057 & 00047 & 00052 & 00048 & 00067 & 00020 & 00009 & 00007 & 00007\end{array}$ 00001000020000000001000000000100000000000000000000 $000000000000000000000009000000 \quad 00000 \quad 000000000006491$

CRYSTAL HEIGHT $=03.000000$ IN. CRYSTAL RADIUS $=01.500000$ IN. $I=10000$

0010400037000340004900047000 $0003100037 \quad 00037 \quad 0004100033 \quad 00039000330003400033$ $00028 \quad 00039000290003300046 \quad 00050 \quad 00053 \quad 0004100033$ $00046 \quad 0004400048 \quad 000440003900058 \quad 00041 \quad 00040 \quad 00043$ $00048 \quad 000390004200041000590004300053 \quad 00056 \quad 00047$ $\begin{array}{llllllllll}00052 & 00065 & 00046 & 00050 & 00053 & 00050 & 00058 & 00047 & 00059 \\ 00054 & 00071 & 00059 & 00064 & 00077 & 00065 & 00080 & 00072 & 00091\end{array}$ 000050000400002000030000200002

INCIDENT ENERGY $=01.330000 \mathrm{MEV}$ SOURCE HEIGHT $=03.936992$ IN. $P=07786$

$A=04765$

00072000910008900072 0000000000000000000000000 $\begin{array}{llllllll}00038 & 00044 & 00035 & 00037 & 00037 & 00044 & 00038\end{array}$ $00042000320004200040 \quad 000420004500039$ $0003500038 \quad 0004700041000390003200041$ $00033000440004400043 \quad 00046 \quad 0004500032$ $\begin{array}{lllllllll}0.0041 & 00043 & 00054 & 00047 & 00057 & 00076 & 00038\end{array}$ $0005700056 \quad 00067000540006700043 \quad 00049$ $00000 \quad 00000 \quad 0003900000 \quad 04765$ 
CRYSTAL HEIGHT $=03.000000$ IN. CRYSTAL RADIUS $=01.500000$ IN. $I=10000$

$00042000260002100026 \quad 0003200030 \quad 00030 \quad 0002800034$

000420002700028000320002800033001

000310003600049000360003000034000

000540003600033000310004100045000

000620006100053000610006400058000

$00048 \quad 00050 \quad 00071000700008100072$

001080013600099001140007300035
INCIDENT ENERGY $=02.620000 \mathrm{MEV}$ SOURCE HEIGHT $=03.936992$ IN. $P=10279$ $\begin{array}{lllllllllll}00030 & 00028 & 00034 & 00039 & 00033 & 00040 & 00031 & 00027 & 00022 & 00028\end{array}$ 00034000310002400027000330003500041000410003000028 $\begin{array}{lllllllllll}00032 & 00038 & 00026 & 00038 & 00035 & 00028 & 00040 & 00042 & 00041 & 00041\end{array}$ $\begin{array}{lllllllllll}00028 & 00030 & 00037 & 00038 & 00042 & 00037 & 00045 & 00035 & 00038 & 00035\end{array}$ $0002900045 \quad 00033 \quad 00048 \quad 00048 \quad 00051 \quad 0004500051 \quad 0023500064$ $0006200058 \quad 00077 \quad 00070 \quad 0006300068 \quad 00055000630006200056$ $\begin{array}{lllllllllll}0063 & 00670 & 00099 & 00104 & 00110 & 00118 & 00102 & 00111 & 00102 & 00105\end{array}$ 00021000120001000003000020000500004000030003703312
CRYSTAL HEIGHT $=03.000000$ IN.
CRYSTAL RADIUS $=01.500000$ IN.

CRYSTAL HEIGHT $=03.000000$ IN.
CRYSTAL RADIUS $=01.500000$ IN. $I=10000$

000250001600014000190001600018000220001700023 $00030 \quad 00018 \quad 00020 \quad 0002400030 \quad 00030 \quad 000200001900020$ $00030 \quad 0002100030 \quad 0002000018 \quad 00021000300002700028$ $0001700020 \quad 00030 \quad 000300003000019000240003900020$ $00018 \quad 000350002700029000250003600034000360003600$ $\begin{array}{llllllllll}00027 & 00045 & 00024 & 00029 & 00049 & 00046 & 00038 & 00043 & 00034 & 000 \\ 00038 & 00054 & 00456 & 00115 & 00109 & 00104 & 00118 & 00106 & 00109 & 000\end{array}$ $\begin{array}{lllllllllll}0 & 0114 & 01548 & 00143 & 00178 & 00158 & 00181 & 00128 & 00142 & 00173 & 00\end{array}$
INCIDENT ENERGY $=04.450000 \mathrm{MEV}$. SOURCE HEIGHT $=03.936992 \mathrm{IN}$. $P=11140$ $\begin{array}{rllllll}00023 & 00016 & 00023 & 00014 & 00019 & 00021 & 00024 \\ 00031 & 00031 & 00021 & 00024 & 00028 & 00025 & 00025\end{array}$ $0003300038 \quad 00043 \quad 000250003100026 \quad 00028$ $0002600040 \quad 0002900029000290003400029$ $00036 \quad 00032 \quad 00036 \quad 0004100023 \quad 00038 \quad 00033$ 00044000330003900044000480003600051 $\begin{array}{lllllllll}00098 & 00098 & 00093 & 00071 & 00067 & 00072 & 00068\end{array}$ 00072000850005200016000020000202485
CRYSTAL HEIGHT $=03.000000$ IN. CRYSTAL RADIUS $=01.500000$ IN. $I=10000$ $000070001200017000120002100016 \quad 000180001700021000190001800015000110002400023 \quad 00012$ $\begin{array}{lllllllllllllllll}0 & 00011 & 00019 & 00016 & 00019 & 00013 & 00021 & 00019 & 00022 & 00013 & 00026 & 00025 & 00027 & 00025 & 00015 & 00020\end{array}$

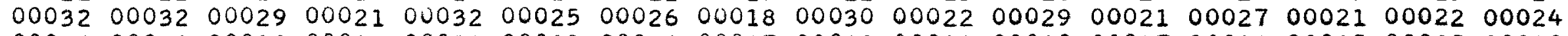
$\begin{array}{llllllllllllllllll}00026 & 00028 & 00023 & 00043 & 00034 & 00031 & 00022 & 00027 & 00032 & 00014 & 00030 & 00027 & 00023 & 00035 & 00035 & 00020\end{array}$ $000320001900036 \quad 00035000310003300038 \quad 00038 \quad 0003000030000360003800039000300003400046$

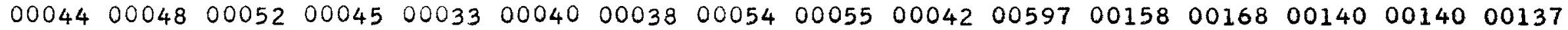

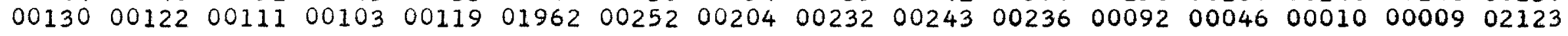

INCIDENT ENERGY $=06.130000 \mathrm{MEV}$ SOURCE HEIGHT = 03.936992 IN. $P=10959$ 00015000110001700019000140002100008000120001800009 